\title{
Variational Sequences, Representation Sequences and Applications in Physics ${ }^{\star}$
}

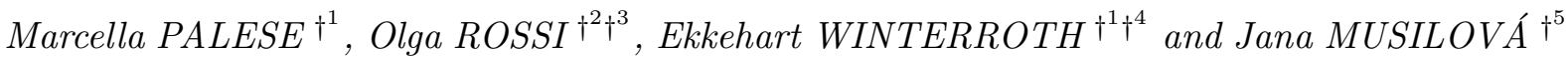 \\ $\dagger^{1}$ Department of Mathematics, University of Torino, via C. Alberto 10, 10123 Torino, Italy \\ E-mail: marcella.palese@unito.it,ekkehart.winterroth@unito.it \\ $\dagger^{2}$ Department of Mathematics, Faculty of Science, University of Ostrava, \\ Ostrava, Czech Republic \\ E-mail: olga.rossi@osu.cz \\ $\dagger^{3}$ Department of Mathematics and Statistics, La Trobe University, Melbourne, Australia \\ $\dagger^{4}$ Lepage Research Institute, 783 42 Slatinice, Czech Republic \\ $\dagger^{5}$ Institute of Theoretical Physics and Astrophysics, Masaryk University Brno, Czech Republic \\ E-mail: janam@physics.muni.cz
}

Received September 22, 2015, in final form April 26, 2016; Published online May 02, 2016

http://dx.doi.org/10.3842/SIGMA.2016.045

\begin{abstract}
This paper is a review containing new original results on the finite order variational sequence and its different representations with emphasis on applications in the theory of variational symmetries and conservation laws in physics.
\end{abstract}

Key words: fibered manifold; jet space; Lagrangian formalism; variational sequence; variational derivative; cohomology; symmetry; conservation law

2010 Mathematics Subject Classification: 55N30; 55R10; 58A12; 58A20; 58E30; 70S10

\section{Introduction}

After Élie Cartan, who in 1922 introduced differential geometric methods into the calculus of variations using the celebrated Cartan form to formulate the variational principle [10], Théophile Lepage in his pioneer work $[73,74]$ initiated the study of the calculus of variations for field theories (multiple integrals) as a theory of differential forms and their exterior differential modulo contact forms (congruences). The main advantage of such an approach evidently is a possibility of framing the calculus of variations within the de Rham sequence of differential forms on a manifold and thus using the de Rham cohomology as an effective tool to study relations between the image and kernel of various differential operators appearing in the calculus of variations.

The attempt to understand global structures of the calculus variations motivated the use of $C$ spectral sequences, and gave rise to the constructions of variational sequences and bicomplexes. This direction was initiated in 1950's by Dedecker $[14,15,16,17,18]$, and continued in the work of Horndeski [41], Gelfand and Dikii [31], Olver and Shakiban [79], Dedecker and Tulczyjew [19], Tulczyjew [96, 97, 98], Takens [93], Anderson [2], Anderson and Duchamp [3], Dickey [20], Vinogradov [99, 100, 101], and others. A main motivation for this research was

- to find solution of the inverse problem of the calculus of variations which means to find local and global constraints for a system of ordinary or partial differential equations to

\footnotetext{
${ }^{\star}$ This paper is a contribution to the Special Issue on Analytical Mechanics and Differential Geometry in honour of Sergio Benenti. The full collection is available at http://www.emis.de/journals/SIGMA/Benenti.html
} 
come from a variational principle as Euler-Lagrange equations, and to construct a Lagrangian,

- to determine the local and global structure of variationally trivial Lagrangians, i.e., Lagrangians giving rise to identically zero Euler-Lagrange expressions (the kernel of the EulerLagrange operator),

- to understand the form and origin of different "canonically looking" expressions appearing in the calculus of variations, as for example

$$
\begin{array}{ll}
\frac{\partial L}{\partial y^{\sigma}}-d_{j} \frac{\partial L}{\partial y_{j}^{\sigma}}+d_{j} d_{k} \frac{\partial L}{\partial y_{j k}^{\sigma}}-\cdots, & \frac{\partial L}{\partial y_{p}^{\sigma}}-d_{j} \frac{\partial L}{\partial y_{j p}^{\sigma}}+d_{j} d_{k} \frac{\partial L}{\partial y_{j k p}^{\sigma}}-\cdots, \\
\frac{\partial E_{\sigma}}{\partial q_{j}^{\nu}}-\sum_{k=j}^{s}(-1)^{k}\left(\begin{array}{c}
k \\
j
\end{array}\right) \frac{d^{k-j}}{d t^{k-j}} \frac{\partial E_{\nu}}{\partial q_{k}^{\sigma}}, & \sum_{l=0}^{s-j-1}(-1)^{j+l}\left(\begin{array}{c}
j+l \\
l
\end{array}\right) \frac{d^{l}}{d t^{l}} \frac{\partial E_{\sigma}}{\partial q_{j+l+1}^{\nu}}
\end{array}
$$

- to understand local and global phenomena typical for variational problems given by local data, as, for instance examples of global Euler-Lagrange forms which do not admit a global Lagrangian; the best known examples are the Galilean relativistic mechanics, or Chern-Simons field theories (where a global Lagrangian exists but is not gaugeinvariant).

The theory was first formulated on infinite order jets. After several attempts to obtain an alternative construction using finite order structures (see, e.g., [3]), the finite order variational sequence was discovered by Krupka 25 years ago [49]. The inspiration originated from the Lepage's idea of a 'congruence', indicating that there should be a close relationship between the Euler-Lagrange operator and the exterior derivative of differential forms, and resulted in the concept of a finite order (cohomological) exact sequence of forms, in which the Euler-Lagrange mapping is included as one arrow.

Compared with the other approaches, the concept of Krupka's variational sequence is conceptually very simple due to the following reasons:

- it uses only finite order jets,

- it appears as a quotient sequence of the de Rham sequence.

This makes it quite easily accessible to potential users, since factorization is a natural, straightforward, commonly understood, and well-mastered technique. Moreover, the factorization procedure can be incorporated into intrinsic operators which allow to avoid tedious and technically difficult calculations.

It should be stressed, however, that here is an additional benefit of the setting on finite order jets: Namely, it provides precise results on the order of the local and global objects of interest.

The aim of this paper is to provide the reader a comprehensive source of the current theory of the finite order variational sequences as it stands 25 years after its beginnings. While for the variational bicomplex theory there is a standard reference the (unpublished) book by Anderson [2], the results on the finite order variational sequence up to now have not been collected, revised and completed in a self-contained article. However, our aim is not merely to provide a revised exposition of known results. Apart from putting known results into a unified treatment, we add a number of original results in order to develop the theory and make it complete. Our aim is also to stimulate the use and applications of the variational sequence in physics. As a motivation we mention a few applications, some of which are interesting and not widely known or even are original here.

The paper is organized as follows: Section 2 introduces the variational sequence as a quotient sequence of the de Rham sequence, developed by Krupka [49, 51, 52, 53] (see also [36, 37, 54, 55]). 
Section 3 is the core of the paper, and deals with the problem of representation of the variational sequence by differential forms. In the variational sequence, the objects are classes of local differential forms. However (as pointed out already by Krupka) the classes can be represented by (global) differential forms: Lagrangians or Lepage $n$-forms (where $n$ is the number of independent variables in the variational problem), Euler-Lagrange forms, Helmholtz forms. This property initiated the search of representation operators and representations with help of differential forms. We present two different representation sequences: the Takens representation sequence based on the so-called interior Euler operator [2] which assigns to every class in the $q$-th column of the variational sequence a source form of degree $q$ (a Lagrangian if $q=n$, a dynamical form if $q=n+1$, a Helmholtz-like form if $q=n+2$ ), and the Lepage representation sequence. The latter is a sequence of Lepage forms (Lepage equivalents of source forms), such that the morphisms are just the exterior derivatives. The existence of such a sequence clarifies the relationship of the variational morphisms to the exterior derivative operator. Moreover, it transfers the questions on the structure of the kernel and image of all the variational morphisms just to application of the Poincaré lemma. A complete representation of the variational sequence by source forms (of any degree) was given by Musilová and Krbek [43, 44, 45] and independently by Krupka and his collaborators [61, 63, 103]. Next, in Section 3 we recall the concept of Lepage form $([46,61,64,72]$ and introduce the Lepage representation of the variational sequence. As a new result concerning Lepage $n$-forms, we present an intrinsic formula for the Cartan form (Theorem 3.8, formula (3.6)). Remarkably, our formula explicitly shows that (and explains why) the higher-order Cartan form generically is not global (this fact was a surprise when discovered and demonstrated by a counterexample in 1980's [40], and up to now has not been well understood). In the subsection on Lepage forms of higher degrees (and any order) we present for the first time properties of general Lepage forms, and namely we give a general intrinsic formula for a Lepage $(n+k)$-form of any order. We also find a higher degree generalization of the famous Cartan form. The techniques based on the use of the interior Euler operator and the residual operator not only lead to elegant formulas and easy proofs; they also enable to avoid lengthy, tedious, and sometimes even impossible calculations in coordinates. The results then help us to find a complete correct Lepage representation of the variational sequence. The last remark in Section 3 concerns the variational order of objects in the variational sequence. We explain how due to the finite order setting it is possible to make (local and global) conclusions on a precise order of Lagrangians and other objects in the variational sequence, distinguishing it from alternative constructions on infinite order jets.

In Section 4 we are concerned with the Lie derivative in the variational sequence. We explore the important property that the Lie derivative of differential forms with respect to prolongations of projectable vector fields preserves the contact structure. This suggests that a Lie derivative of classes of forms, a variational Lie derivative, can be correctly defined as the equivalence class of the standard Lie derivative of forms and represented by forms. New results in this section are then contained in subsequent theorems generalizing the first variation formula to forms of any degree.

The last section of the paper is devoted to selected applications of the variational sequence theory. We recall the fundamental well-known applications to the inverse variational problem and to variationally trivial Lagrangians. Apart from that we emphasize some other possible applications which have not yet been fully explored - like, for instance, properties of Helmholtz forms. We include also examples of variational problems defined by local data, and on symmetries and conservation laws, emphasizing cohomological questions and local and global phenomena.

For a reader interested in a wider context of the theory of variational sequences, we refer to the survey works $[54,102]$, and to the book [55]. 
M. Palese, O. Rossi, E. Winterroth and J. Musilová

\section{The variational sequence}

\section{$2.1 \quad$ Jet bundles and contact forms}

Fibred manifolds and their jet prolongations represent a convenient mathematical framework for mechanics and field theories, suitable for investigation of Lagrangian systems of different orders, and one or many independent variables within a sufficiently general and unified geometric framework. For an excellent exposition of the jet bundle geometry we refer to the book [91].

In what follows, we shall consider a smooth fibred manifold $\pi: \boldsymbol{Y} \rightarrow \boldsymbol{X}$, with $\operatorname{dim} \boldsymbol{X}=n$ and $\operatorname{dim} \boldsymbol{Y}=n+m$, and its $r$-jet prolongations $\pi_{r}: J^{r} \boldsymbol{Y} \rightarrow \boldsymbol{X}$, where $r \geq 1$. For $n=1$ the manifold $\boldsymbol{Y}$ is a space of events for mechanical systems of $m$ degrees of freedom, and local sections of $\pi$ are graphs of curves, so usually $\boldsymbol{X}=\mathbb{R}$ (meaning time, or just a parameter for the curves). If $n>1$ then $\boldsymbol{X}$ has the meaning of a physical space or spacetime, and local sections of $\pi$ are physical fields over the manifold $\boldsymbol{X}$. Then $J^{r} \boldsymbol{Y}$ is a manifold of points $j_{x}^{r} \gamma$, the equivalence classes of local sections $\gamma$ of $\pi$ with the same value at $x$ and the same partial derivatives at $x$ up to the order $r$. Thus $J^{r} \boldsymbol{Y}$ serves as a domain for functions, differential forms, vector fields, and other objects depending on higher derivatives (up to the $r$-th order).

Due to the affine bundle structure of $\pi_{r+1, r}: J^{r+1} \boldsymbol{Y} \rightarrow J^{r} \boldsymbol{Y}$, we have a natural splitting $J^{r} \boldsymbol{Y} \times{ }_{J^{r-1} \boldsymbol{Y}} T^{*} J^{r-1} \boldsymbol{Y}=J^{r} \boldsymbol{Y} \times{ }_{J^{r-1}} \boldsymbol{Y}\left(T^{*} \boldsymbol{X} \oplus V^{*} J^{r-1} \boldsymbol{Y}\right)$ which induces natural splittings into horizontal and vertical parts of projectable vector fields, forms, and of the exterior differential on $J^{r} \boldsymbol{Y}$.

A differential $q$-form $\omega$ on $J^{r} \boldsymbol{Y}$ is called contact if $J^{r} \gamma^{*} \omega=0$ for every local section $\gamma$ of $\pi$. Contact forms on $J^{r} \boldsymbol{Y}$ form an ideal in the exterior algebra, called the contact ideal of order $r$. The contact ideal is generated by contact 1-forms and their exterior derivatives [50]. A differential $q$-form $\omega$ on $J^{r} \boldsymbol{Y}$ is called horizontal, or 0 -contact, if $\left.\xi\right\rfloor \omega=0$ for every vector field $\xi$ on $J^{r} \boldsymbol{Y}$, vertical with respect to the projection onto $\boldsymbol{X}$.

From the definitions it follows that every $q$-form for $q>n$ is contact, and that every form $\omega$ on $J^{r} \boldsymbol{Y}$, if lifted to $J^{r+1} \boldsymbol{Y}$, is a sum of a unique horizontal and contact form. This splitting can be further refined if the concept of a $k$-contact form for $k \geq 1$ is introduced. A contact form $\omega$ is called $k$-contact if for every vertical $\xi$ the contraction $\xi\rfloor \omega$ is $(k-1)$-contact. Then, as proved in [48], every $q$-form $\omega$ on $J^{r} \boldsymbol{Y}, q \geq 1$, admits the unique decomposition

$$
\pi_{r+1, r}^{*} \omega=\sum_{k=0}^{q} p_{k} \omega
$$

into a sum of $k$-contact forms, $0 \leq k \leq q$. For the horizontal (0-contact) operator often the notation $h=p_{0}$ is used. The form $p_{k} \omega$ is called the $k$-contact component of $\omega$. Note that if $q \geq n+1$ then $\omega$ is contact, and it is at least $(q-n)$-contact, i.e., the contact components $p_{0}, p_{1}, \ldots, p_{q-n-1}$ of $\omega$ are equal to zero. If, moreover, $p_{q-n} \omega=0$, we speak about a strongly contact form [49].

An important special case of the decomposition (2.1) concerns closed forms and their local primitives, obtained due to the Poincaré lemma, by means of the Poincaré homotopy operator $\mathcal{P}$. Since every form $\omega$ can be locally expressed as $\omega=\mathcal{P} d \omega+d \mathcal{P} \omega$, equation $\omega=d \rho$ has a local solution $\rho=\mathcal{P} \omega$. Applying, however, the decomposition into contact components we can see that there arises a modified homotopy operator, denoted by $\mathcal{A}$, and called the contact homotopy operator [48], which is adapted to the decomposition (2.1) and defined by $\mathcal{A} p_{0} \omega=0$, $\mathcal{A} p_{k} \omega=p_{k-1} \mathcal{P} \omega, k \geq 1$. It satisfies $\pi_{r+1, r}^{*} \omega=\mathcal{A} d\left(\pi_{r+1, r}^{*} \omega\right)+d \mathcal{A}\left(\pi_{r+1, r}^{*} \omega\right)$, and moreover, if (locally) $\omega=d \rho$, then

$$
\pi_{r+1, r}^{*} \rho=\mathcal{A}\left(\pi_{r+1, r}^{*} \omega\right)=\sum_{k=0}^{q} \mathcal{A} p_{k+1} \omega,
$$


hence $p_{k} \rho=\mathcal{A} p_{k+1} \omega$. Compared to $\mathcal{P}$, the operator $\mathcal{A}$ concerns vertical curves (curves in the fibres over $\boldsymbol{X}$ ) only.

Notice that the decomposition (2.1) applied to $\omega=d \rho$ induces a splitting of the exterior derivative $d$ to $\pi_{r+1, r}^{*} d=d_{H}+d_{V}$ where the horizontal derivative $d_{H}=h d$, and the vertical derivative $d_{V}$ is the contact part of $\pi_{r+1, r}^{*} d$.

The splitting of vector fields then arises with help of the total derivative operator. If we denote $\left(x^{i}, y^{\sigma}\right)$ local fibred coordinates on $\boldsymbol{Y}$, and $\left(x^{i}, y_{J}^{\sigma}\right)$ where $J$ is a multiindex, $0 \leq|J| \leq r$, the associated coordinates on $J^{r} \boldsymbol{Y}$, then the $i$-th total (formal) derivative takes the form

$$
d_{i}=\frac{\partial}{\partial x^{i}}+\sum_{|J|=0}^{r} y_{J i}^{\sigma} \frac{\partial}{\partial y_{J}^{\sigma}}
$$

Considered as a vector field along the projection $\pi_{r+1, r}$, the total derivative induces a splitting of vector fields to a horizontal and vertical part as follows: Letting

$$
\xi=\xi^{i} \frac{\partial}{\partial x^{i}}+\sum_{|J|=0}^{r} \xi_{J}^{\sigma} \frac{\partial}{\partial y_{J}^{\sigma}}
$$

be a vector field on $J^{r} \boldsymbol{Y}$, in order to obtain the splitting $\xi=\xi_{H}+\xi_{V}$, we put

$$
\xi_{H}=\xi^{i} d_{i}, \quad \xi_{V}=\xi-\xi_{H}=\sum_{|J|=0}^{r}\left(\xi_{J}^{\sigma}-y_{J i}^{\sigma} \xi^{i}\right) \frac{\partial}{\partial y_{J}^{\sigma}} .
$$

Note that both $\xi_{H}$ and $\xi_{V}$ are vector fields along the projection $\pi_{r+1, r}$ rather than 'ordinary' vector fields on $J^{r} \boldsymbol{Y}$. If $\Xi$ is a projectable vector field on $\boldsymbol{Y}$, we write $J^{r} \Xi_{V}=\left(J^{r} \Xi\right)_{V}$ (this can be viewed as a definition of prolongation of $\Xi_{V}$ which is a vector field along the projection $\left.\pi_{1,0}\right)$.

Recall that $\mathcal{C}\left(\pi_{r}\right)$, the contact distribution or Cartan distribution of order $r$, is locally annihilated by contact 1 -forms

$$
\omega_{J}^{\sigma}=d y_{J}^{\sigma}-y_{J j}^{\sigma} d x^{j}, \quad 1 \leq \sigma \leq m,
$$

or, equivalently, locally generated by the vector fields $d_{i}$ along $\pi_{r, r-1}$ and $\partial / \partial y_{J}^{\sigma}$ where $|J|=r$. The Cartan distribution on $J^{r} \boldsymbol{Y}$ is not completely integrable, hence the ideal generated by contact 1-forms of order $r$ is not closed. Its closure is then the contact ideal of order $r$ (the ideal of all contact forms on $J^{r} \boldsymbol{Y}$ ).

In what follows, we shall need on $J^{r} \boldsymbol{Y}$ the ideal generated by lifts of contact 1-forms of order 1 , whose elements take the form $\pi_{r, 1}^{*} \omega \wedge \eta$ where $\omega \in \mathcal{C}^{0}\left(\pi_{1}\right)$ (the annihilator of $\mathcal{C}\left(\pi_{1}\right)$ ) and $\eta$ is of order $r$. Since locally they are expressed as $\omega^{\sigma} \wedge \eta_{\sigma}$, where $\omega^{\sigma}=d y^{\sigma}-y_{j}^{\sigma} d x^{j}$, $1 \leq \sigma \leq m$, are the basic local contact 1-forms of order 1 , we say that forms belonging to this ideal are $\omega^{\sigma}$-generated.

In the calculus of variations and the theory of differential equations on fibred manifolds, the fundamental role is played by the sheaf $\Lambda_{n, \boldsymbol{X}}^{r}$ of horizontal (0-contact) $n$-forms on $J^{r} \boldsymbol{Y}$, the elements of which are called Lagrangians of order $r$, and the sheaf $\Lambda_{n+1,1, \boldsymbol{Y}}^{r}$ of 1-contact $(n+1)$ forms on $J^{r} \boldsymbol{Y}$, horizontal with respect to the projection onto $\boldsymbol{Y}$, the elements of which are called dynamical forms of order $r$. By means of variation of the action defined by a Lagrangian $\lambda$ one obtains a distinguished dynamical form $E_{\lambda}$, called the Euler-Lagrange form of $\lambda$ [46]; the components of $E_{\lambda}$ in every fibred chart are the Euler-Lagrange expressions of the Lagrangian $\lambda$. Remarkably, if $\lambda$ is of order $r$ then $E_{\lambda}$ is of order $\leq 2 r$.

The mapping assigning to every Lagrangian its Euler-Lagrange form is called the EulerLagrange mapping. 
A dynamical form $E$ is called globally variational if there exists a Lagrangian $\lambda$ such that (possibly up to a jet projection) $E=E_{\lambda}$. $E$ is called locally variational if every point in the domain of $E$ has a neighbourhood $U$ where $E$ is variational [48].

Dynamical forms are often called source forms, as originally introduced by Takens [93]. However, we shall follow Krupková and Prince [70] who extended the concept of source form to forms of all degrees $q=n+k$ where $k \geq 1$ :

Definition 2.1. Let $k \geq 1$. By a source form of degree $n+k$ we shall mean a $\omega^{\sigma}$-generated $k$-contact $(n+k)$-form.

For $k=1$, dynamical forms are $\omega^{\sigma}$-generated 1-contact $(n+1)$-forms, hence, indeed, source forms of degree $n+1$. Source forms of degree $n+2$ are called Helmholtz-like forms [69]. For the calculus of variations the most important examples of source forms of degree $n+1$ are Euler-Lagrange forms, and of source forms of degree $n+2$ the Helmholtz forms.

\subsection{The variational sequence}

Now we recall the finite order variational sequence as introduced by Krupka in [49]. The concept uses the sheaf theory (standard references are, e.g., [8, 104]). However, for a first understanding, a concrete use of the variational sequence, and calculations it is not necessary to be familiar with all the rather difficult mathematical theory. In place of a 'sheaf' of forms in this context one can roughly imagine a family of modules of local differential forms defined on the open subsets of the corresponding manifold.

In what follows, we denote by $\mathbb{R}_{\boldsymbol{Y}}$ the constant sheaf over $\mathbb{R}$, and by $\Lambda_{q}^{r}$ the sheaf of $q$-forms on $J^{r} \boldsymbol{Y}$. We let $\Lambda_{0, c}^{r}=\{0\}$, and $\Lambda_{q, c}^{r}$ be the sheaf of contact $q$-forms, if $q \leq n$, or the sheaf of strongly contact $q$-forms, if $q>n$, on $J^{r} \boldsymbol{Y}$. We set

$$
\Theta_{q}^{r}=\Lambda_{q, c}^{r}+\left(d \Lambda_{q-1, c}^{r}\right),
$$

where $d \Lambda_{q-1, c}^{r}$ is the image of $\Lambda_{q-1, c}^{r}$ by the exterior derivative $d$, and $\left(d \Lambda_{q-1, c}^{r}\right)$ denotes the sheaf generated by the presheaf $d \Lambda_{q-1, c}^{r}$. We note that $\Theta_{q}^{r}=0$ for $q>\operatorname{corank} \mathcal{C}\left(\pi_{r}\right)$.

The subsequence

$$
0 \rightarrow \Theta_{1}^{r} \rightarrow \Theta_{2}^{r} \rightarrow \Theta_{3}^{r} \rightarrow \cdots
$$

of the de Rham sequence

$$
0 \rightarrow \mathbb{R}_{\boldsymbol{Y}} \rightarrow \Lambda_{0}^{r} \rightarrow \Lambda_{1}^{r} \rightarrow \Lambda_{2}^{r} \rightarrow \Lambda_{3}^{r} \rightarrow \cdots
$$

is an exact sequence of soft sheaves. The quotient sequence

$$
0 \rightarrow \mathbb{R}_{\boldsymbol{Y}} \rightarrow \Lambda_{0}^{r} \rightarrow \Lambda_{1}^{r} / \Theta_{1}^{r} \rightarrow \Lambda_{2}^{r} / \Theta_{2}^{r} \rightarrow \Lambda_{3}^{r} / \Theta_{3}^{r} \rightarrow \cdots
$$

is called the variational sequence of order $r$. The main theorem due to Krupka [49] then states that the variational sequence is an acyclic resolution of the constant sheaf $\mathbb{R}_{\boldsymbol{Y}}$ (meaning that the sequence is locally exact with the exception of $\mathbb{R}_{\boldsymbol{Y}}$ ). Hence, due to the abstract de Rham theorem, the cohomology groups of the cochain complex of global sections of the variational sequence are identified with the de Rham cohomology groups $H_{\mathrm{dR}}^{q} \boldsymbol{Y}$ of the manifold $\boldsymbol{Y}$.

It should be stressed that objects in the variational sequence are elements of the quotient sheaves $\Lambda_{q}^{r} / \Theta_{q}^{r}$, i.e., they are equivalence classes of local $r$-th order differential $q$-forms. We denote by $[\rho] \in \Lambda_{q}^{r} / \Theta_{q}^{r}$ the class of $\rho \in \Lambda_{q}^{r}$. Morphisms in the variational sequence are then by construction quotients of the exterior derivative $d$. We denote

$$
\mathcal{E}_{q}: \Lambda_{q}^{r} / \Theta_{q}^{r} \rightarrow \Lambda_{q+1}^{r} / \Theta_{q+1}^{r},
$$


so that

$$
\mathcal{E}_{q}([\rho])=[d \rho],
$$

and $\mathcal{E}_{q}([\rho])=[d \rho]=0$ means that there exists $[\eta] \in \Lambda_{q-1}^{r} / \Theta_{q-1}^{r}$ such that $[\rho]=\mathcal{E}_{q-1}([\eta])=[d \eta]$. If, moreover, $H_{\mathrm{dR}}^{q} \boldsymbol{Y}=\{0\}$ then the class $[\eta]$ has a global representative.

The meaning of this abstract construction for the calculus of variations becomes clear in view of the following theorem:

Theorem 2.2 (Krupka $[49,54]$ ). The sheaf $\Lambda_{n}^{r} / \Theta_{n}^{r}$ is isomorphic with a subsheaf of the sheaf of Lagrangians $\Lambda_{n, \boldsymbol{X}}^{r+1}$, the sheaf $\Lambda_{n+1}^{r} / \Theta_{n+1}^{r}$ is isomorphic with a subsheaf of the sheaf of source forms $\Lambda_{n+1,1, \boldsymbol{Y}}^{2 r+1}$, and the quotient mapping $\mathcal{E}_{n}: \Lambda_{n}^{r} / \Theta_{n}^{r} \rightarrow \Lambda_{n+1}^{r} / \Theta_{n+1}^{r}$ is the Euler-Lagrange mapping.

By this theorem, the elements of the quotient sheaf $\Lambda_{n}^{r} / \Theta_{n}^{r}$ have the meaning of Lagrangians. The kernel of $\mathcal{E}_{n}$ then consists of null-Lagrangians (variationally trivial Lagrangians), and its image, which, by exactness is the kernel of the next morphism,

$$
\mathcal{E}_{n+1}: \Lambda_{n+1}^{r} / \Theta_{n+1}^{r} \rightarrow \Lambda_{n+2}^{r} / \Theta_{n+2}^{r},
$$

represents variational equations. Therefore $\mathcal{E}_{n+1}$ is called Helmholtz mapping. The name refers to the famous "Helmholtz conditions" [39], necessary and sufficient conditions for differential equations to be variational (as they stand). Remarkably, the Helmholtz morphism, discovered within the variational sequence theory, has not been known in the classical calculus of variations.

The above theorem also suggests the idea that all classes in the variational sequence could be representable by differential forms with a clear place in the calculus of variations. So far there have been noticed two such representation sequences: the "source form representation" which we call Takens representation, and the "Lepage form representation". In the former the sheaves contain familiar differential forms which appear in the calculus of variations, like Lagrangians and Euler-Lagrange forms, and their generalizations, like, for example, Helmholtz forms. The morphisms are then the corresponding variational mappings, like, e.g., the Euler-Lagrange mapping sending Lagrangians to Euler-Lagrange forms, or the Helmholtz mapping, sending dynamical forms to Helmholtz forms. The latter representation has as objects Lepage forms, which are extensions of the "familiar forms" mentioned above by higher contact components in such a way that the morphisms are the exterior derivatives, so that in the Lepage form representation the variational sequence "morally" (up to the orders of individual subsheaves) becomes a subsequence of the de Rham sequence. We shall deal with the representation sequences below.

In what follows, we shall denote

$$
\mathcal{V}_{0}^{r}=\Lambda_{0}^{r}, \quad \mathcal{V}_{q}^{r}=\Lambda_{q}^{r} / \Theta_{q}^{r},
$$

and the variational sequence of order $r$ will be shortly written in the form

$$
0 \rightarrow \mathbb{R}_{\boldsymbol{Y}} \rightarrow \mathcal{V}_{*}^{r}
$$

\section{Representation sequences}

\subsection{The interior Euler operator}

Let us introduce an operator which plays an essential role in the representation theory for the variational sequence. It was introduced to the calculus of variations within the variational bicomplex theory in $[2,4]$, and adapted to the finite order situation of the variational sequence in $[43,44,45,61]$. This operator, called interior Euler operator, and denoted by $\mathcal{I}$, reflects in 
an intrinsic way the procedure of getting a distinguished representative of a class $[\rho] \in \Lambda_{q}^{r} / \Theta_{q}^{r}$ for $q>n$ by applying to $\rho$ the operator $p_{q-n}$ and a factorization by $\Theta_{q}^{r}$.

Let $\left(x^{i}, y^{\sigma}\right)$ denote local fibred coordinates on $\boldsymbol{Y}$, and $\left(x^{i}, y_{J}^{\sigma}\right)$ the associated coordinates on $J^{r} \boldsymbol{Y}$, where $J$ is a multiindex, $0 \leq|J| \leq r, J=\left(j_{1}, \ldots, j_{p}\right)$ with $1 \leq j_{1} \leq j_{2} \leq \cdots \leq j_{p} \leq n$ and $p \leq r$.

Let $k \geq 1$ and $q=n+k$. Recall that if $\rho \in \Lambda_{n+k}^{r}$, we have

$$
\pi_{r+1, r}^{*} \rho=p_{k} \rho+p_{k+1} \rho+\cdots+p_{k+n} \rho .
$$

Following [45] we set

$$
\left.\mathcal{I}(\rho)=\frac{1}{k} \omega^{\sigma} \wedge \sum_{|J|=0}^{r}(-1)^{|J|} d_{J}\left(\partial / \partial y_{J}^{\sigma}\right\rfloor p_{k} \rho\right),
$$

where $d_{J}=d_{j_{1}} \ldots d_{j_{p}}$ for $|J|=p$. It can be shown by means of the partition of unity arguments that this formula defines a global form $\mathcal{I}(\rho)$.

The operator $\mathcal{I}: \Lambda_{n+k}^{r} \rightarrow \Lambda_{n+k}^{2 r+1}$ has the following properties:

(1) for every $\rho \in \Lambda_{n+k}^{r}, \mathcal{I}(\rho)$ is a source form of degree $n+k$,

(2) $\mathcal{I}\left(p_{k} \rho\right)=\mathcal{I}(\rho)$

(3) $\mathcal{I}(\rho)$ belongs to the same class as $\pi_{2 r+1, r}^{*} \rho$, i.e., $\pi_{2 r+1, r}^{*} \rho-\mathcal{I}(\rho) \in \Theta_{n+k}^{2 r+1}$,

(4) $\mathcal{I}^{2}=\mathcal{I}$, up to a canonical projection; precisely, $\mathcal{I}^{2}(\rho)=\pi_{4 r+3,2 r+1}^{*} \mathcal{I}(\rho)$,

(5) $\operatorname{Ker} \mathcal{I}=\Theta_{n+k}^{r}$.

By construction, $\mathcal{I}(\rho)$ is a $k$-contact form, so there is a question about the difference between $\mathcal{I}(\rho)$ and $p_{k} \rho$. It turns out that the difference can be expressed intrinsically by means of an operator $\mathcal{R}$ which we shall call residual operator, as follows

$$
p_{k} \rho=\mathcal{I}(\rho)+p_{k} d p_{k} \mathcal{R}(\rho)
$$

(up to appropriate canonical projections). By the properties (2) and (4) of $\mathcal{I}$ we can see that

$$
\mathcal{I}\left(p_{k} d p_{k} \mathcal{R}(\rho)\right)=0 .
$$

$\mathcal{R}(\rho)$ is by definition a local strongly contact $(n+k-1)$-form. Note that, contrary to $\mathcal{I}(\rho)$, the form $\mathcal{R}(\rho)$ need not be unique and need not be global.

Moreover, $\mathcal{I}(\rho)$ is a source form, so there is a question about its relationship with other source forms in the same class $[\rho]$. To clarify this it is useful to introduce the concept of a canonical source form as a source form $\rho$ such that (up to projection) $\rho=\mathcal{I}(\rho)[70]$.

Proposition 3.1. Every source form is equivalent with a canonical source form (of possibly higher order). More precisely, letting $\rho=\omega^{\sigma} \wedge \eta_{\sigma}$ be a source $(n+k)$-form it holds (up to projection)

$$
\rho=k \mathcal{I}(\rho)-(k-1) \omega^{\sigma} \wedge \mathcal{I}\left(\eta_{\sigma}\right)
$$

and

$$
\rho-\mathcal{I}(\rho)=p_{k} d p_{k} \mathcal{R}(\rho)=\frac{k-1}{k} \omega^{\sigma} \wedge p_{k-1} d p_{k-1} \mathcal{R}\left(\eta_{\sigma}\right) .
$$

As a consequence, for $k \geq 2$,

$$
\mathcal{I}\left(\omega^{\sigma} \wedge \eta_{\sigma}\right)=\mathcal{I}\left(\omega^{\sigma} \wedge \mathcal{I}\left(\eta_{\sigma}\right)\right) .
$$


Proof. The first assertion is just the property (3) of $\mathcal{I}$. Next two formulas easily follow from the definitions of $\mathcal{I}$ and $\mathcal{R}$ and the fact that $\rho$ is the source form, i.e., $\pi_{r+1, r}^{*} \rho=p_{k} \rho$. Indeed, for $\rho=\omega^{\sigma} \wedge \eta_{\sigma}$ we have

$$
\begin{aligned}
\mathcal{I}\left(\omega^{\sigma} \wedge \eta_{\sigma}\right) & \left.=\frac{1}{k} \omega^{\sigma} \wedge \sum_{|J|=0}^{r}(-1)^{|J|} d_{J}\left(\partial / \partial y_{J}^{\sigma}\right\rfloor\left(\omega^{\nu} \wedge \eta_{\nu}\right)\right) \\
& \left.=\frac{1}{k} \omega^{\sigma} \wedge\left(\eta_{\sigma}-\omega^{\nu} \wedge \sum_{|J|=0}^{r}(-1)^{|J|} d_{J}\left(\partial / \partial y_{J}^{\sigma}\right\rfloor \eta_{\nu}\right)\right)=\frac{1}{k} \omega^{\sigma} \wedge \eta_{\sigma}+\frac{k-1}{k} \omega^{\sigma} \wedge \mathcal{I}\left(\eta_{\sigma}\right) .
\end{aligned}
$$

This formula then yields

$$
p_{k} d p_{k} \mathcal{R}(\rho)=\rho-\mathcal{I}(\rho)=\frac{k-1}{k} \omega^{\sigma} \wedge\left(\eta_{\sigma}-\mathcal{I}\left(\eta_{\sigma}\right)\right)=\frac{k-1}{k} \omega^{\sigma} \wedge p_{k-1} d p_{k-1} \mathcal{R}\left(\eta_{\sigma}\right),
$$

as desired.

Finally, applying $\mathcal{I}$ to the first formula we get $(k-1) \mathcal{I}(\rho)=(k-1) \mathcal{I}\left(\omega^{\sigma} \wedge \mathcal{I}\left(\eta_{\sigma}\right)\right)$, which provides the last formula for $k \geq 2$.

For dynamical forms Proposition 3.1 gives the following important uniqueness result [70].

Corollary 3.2. For $k=1$, every source form is a canonical source form.

Note that this can be seen also directly from the definition of $\mathcal{I}$ : Indeed, if $k=1$ and $\rho$ is a $\omega^{\sigma}$-generated 1 -contact $(n+1)$-form then in (3.1) the contractions give horizontal $n$-forms with the same components as those of $\rho$, and the summation over $|J|$ reduces to one term, $|J|=0$. Hence, $\left.\mathcal{I}(\rho)=\omega^{\sigma} \wedge\left(\partial / \partial y^{\sigma}\right\rfloor \rho\right)=\rho$.

For the description of the image of $\mathcal{I}$ we refer also to [63].

Example 3.3. We shall show the computations of $\mathcal{I}(\rho)$ and $\mathcal{R}(\rho)$ for a second-order 1-contact 2 -form $\rho$ in mechanics $(n=1, k=1, r=2)$.

Consider local fibred coordinates $\left(t, q^{\sigma}\right)$ on $\boldsymbol{Y}$ and the corresponding associated coordinates $\left(t, q^{\sigma}, q_{j}^{\sigma}\right), 1 \leq j \leq r, 1 \leq \sigma \leq m$, on $J^{r} \boldsymbol{Y}$, and denote $d_{t}$ the total derivative operator and $\omega_{j}^{\sigma}=d q_{j}^{\sigma}-q_{j+1}^{\sigma} d t$ the local basic contact 1-forms, and put $\omega^{\sigma}=\omega_{0}^{\sigma}$. The calculation of the image of $\rho$ by the interior Euler operator is direct, using (3.1). We have

$$
p_{1} \rho=A_{\sigma}^{0} \omega^{\sigma} \wedge d t+A_{\sigma}^{1} \omega_{1}^{\sigma} \wedge d t+A_{\sigma}^{2} \omega_{2}^{\sigma} \wedge d t,
$$

hence

$$
\left.\mathcal{I}(\rho)=\omega^{\sigma} \wedge \sum_{j=0}^{r=2}(-1)^{j} d_{t}^{j}\left(\frac{\partial}{\partial q_{j}^{\sigma}}\right\rfloor p_{1} \rho\right)=\left(A_{\sigma}^{0}-d_{t} A_{\sigma}^{1}+d_{t}^{2} A_{\sigma}^{2}\right) \omega^{\sigma} \wedge d t .
$$

The residual term is then

$$
p_{1} d p_{1} \mathcal{R}(\rho)=\pi_{5,3}^{*} p_{1} \rho-\mathcal{I}(\rho)=\left(d_{t} A_{\sigma}^{1}-d_{t}^{2} A_{\sigma}^{2}\right) \omega^{\sigma} \wedge d t+A_{\sigma}^{1} \omega_{1}^{\sigma} \wedge d t+A_{\sigma}^{2} \omega_{2}^{\sigma} \wedge d t .
$$

Let us compute $\mathcal{R}(\rho)$. Since $\mathcal{R}(\rho)$ is a contact 1-form of order two, it holds $\mathcal{R}(\rho)=C_{\sigma}^{0} \omega^{\sigma}+$ $C_{\sigma}^{1} \omega_{1}^{\sigma}+C_{\sigma}^{2} \omega_{2}^{\sigma}=p_{1} \mathcal{R}(\rho)$. The 1-contact part of the exterior derivative is $p_{1} d \mathcal{R}(\rho)=-d_{t} C_{\sigma}^{0} \omega^{\sigma} \wedge$ $d t-\left(d_{t} C_{\sigma}^{1}+C_{\sigma}^{0}\right) \omega_{1}^{\sigma} \wedge d t-\left(d_{t} C_{\sigma}^{2}+C_{\sigma}^{1}\right) \omega_{2}^{\sigma} \wedge d t-C_{\sigma}^{2} \omega_{3}^{\sigma} \wedge d t$. Comparing this with the formula above yields

$$
C_{\sigma}^{2}=0, \quad d_{t} C_{\sigma}^{2}+C_{\sigma}^{1}=-A_{\sigma}^{2}, \quad d_{t} C_{\sigma}^{1}+C_{\sigma}^{0}=-A_{\sigma}^{1}, \quad-d_{t} C_{\sigma}^{0}=d_{t} A_{\sigma}^{1}-d_{t}^{2} A_{\sigma}^{2} .
$$

From here we immediately see that $C_{\sigma}^{1}=-A_{\sigma}^{2}$ and $C_{\sigma}^{0}=-A_{\sigma}^{1}+d_{t} A_{\sigma}^{2}$, and that the equation $-d_{t} C_{\sigma}^{0}=d_{t} A_{\sigma}^{1}-d_{t}^{2} A_{\sigma}^{2}$ is satisfied identically. So finally,

$$
\mathcal{R}(\rho)=\left(d_{t} A_{\sigma}^{2}-A_{\sigma}^{1}\right) \omega^{\sigma}-A_{\sigma}^{2} \omega_{1}^{\sigma} .
$$

We can easily verify that $\pi_{5,3}^{*} p_{1} \rho=\mathcal{I}(\rho)+p_{1} d p_{1} \mathcal{R}(\rho)$. 


\subsection{Takens representation of the variational sequence}

Once we have the interior Euler operator, we can obtain a sequence of sheaves of differential forms (rather than of classes of differential forms), such that both the objects and the morphisms have a straightforward interpretation in the calculus of variations. Such a representation of the variational sequence, introduced by Krbek and Musilová (see [43, 44, 45]) basically concerns source forms (of all degrees), therefore we call it in honour of Takens the Takens representation.

The motivation for the representation comes from the following observations:

- If $q \leq n$ then for every $q$-form $\rho$ of order $r$, the contact decomposition (2.1) takes the form

$$
\pi_{r+1, r}^{*} \rho=h \rho+p_{1} \rho+\cdots+p_{q} \rho,
$$

and $\Theta_{q}^{r}=\Lambda_{q, c}^{r}+\left(d \Lambda_{q-1, c}^{r}\right)$, where we have sheaves of contact forms. This means that given a class $[\rho] \in \mathcal{V}_{q}^{r}=\Lambda_{q}^{r} / \Theta_{q}^{r}$, one has $\rho_{1} \sim \rho_{2}$ iff $\rho_{1}-\rho_{2}=\vartheta+d \eta$ where $\vartheta$ is a contact $q$-form and $\eta$ is a contact $(q-1)$-form, i.e.,

$$
h \rho_{1}=h \rho_{2} .
$$

Hence, every class $[\rho]$ is completely determined by a unique horizontal form, and, in particular, if $q=n$, by a Lagrangian.

Summarizing, for $q \leq n$ the operator of horizontalization,

$$
h: \Lambda_{q}^{r} \rightarrow \Lambda_{q, \boldsymbol{X}}^{r+1} \subset \Lambda_{q}^{r+1}, \quad \rho \rightarrow h \rho,
$$

induces a representation mapping,

$$
R_{q}: \mathcal{V}_{q}^{r} \rightarrow \Lambda_{q}^{r+1}, \quad R_{q}([\rho])=h \rho .
$$

- If $q=n+k$ for $k>1$ then for every $q$-form $\rho$ of order $r$, the contact decomposition (2.1) takes the form

$$
\pi_{r+1, r}^{*} \rho=p_{k} \rho+p_{k+1} \rho+\cdots+p_{q} \rho,
$$

and $\Theta_{q}^{r}=\Lambda_{q, c}^{r}+\left(d \Lambda_{q-1, c}^{r}\right)$, where we have sheaves of strongly contact forms (with the only exception of $\Lambda_{n, c}^{r}$ which is a sheaf of contact forms). This means that given a class $[\rho] \in \mathcal{V}_{n+k}^{r}=$ $\Lambda_{n+k}^{r} / \Theta_{n+k}^{r}$, one has $\rho_{1} \sim \rho_{2}$ iff $\rho_{1}-\rho_{2}=\vartheta+d \eta$ where $\vartheta$ is a strongly contact $(n+k)$-form (i.e., such that $\left.p_{k} \vartheta=0\right)$ and $\eta$ is a strongly contact $(n+k-1)$-form $\left(p_{k-1} \eta=0\right)$. Thus $p_{k} \rho_{1}=p_{k} \rho_{2}+p_{k} d p_{k} \eta \neq p_{k} \rho_{2}$. However, $\rho_{1}-\rho_{2} \in \Theta_{n+k}^{r}$, so that

$$
\mathcal{I}\left(\rho_{1}\right)=\mathcal{I}\left(\rho_{2}\right) \text {. }
$$

Hence, every class $[\rho] \in \mathcal{V}_{n+k}^{r}$ is completely determined by a unique canonical source form.

If we denote by $\mathcal{I} \Lambda_{n+k}^{r}$ the image of $\Lambda_{n+k}^{r}$ by $\mathcal{I}$, we can see that the interior Euler operator

$$
\mathcal{I}: \Lambda_{n+k}^{r} \rightarrow \mathcal{I} \Lambda_{n+k}^{r} \subset \Lambda_{n+k}^{2 r+1}, \quad \rho \rightarrow \mathcal{I}(\rho),
$$

induces a representation mapping,

$$
R_{n+k}: \mathcal{V}_{n+k}^{r} \rightarrow \Lambda_{n+k}^{2 r+1}, \quad R_{n+k}([\rho])=\mathcal{I}(\rho) .
$$

For $k=1$ there is no other source form representing a class $[\rho] \in \mathcal{V}_{n+1}^{r}$, so that every class is completely determined by a unique dynamical form. If $[\rho]=\mathcal{E}_{n}(\mu)=[d \mu]$ then $\mathcal{I}(d \mu)=\mathcal{I}(d h \mu)$ is the Euler-Lagrange form of the Lagrangian $h \mu$. Note that this argument proves the uniqueness of the Euler-Lagrange form. At the same time Proposition 3.1 shows us that for $k \geq 2$ the 
representing canonical source form is no longer a unique source form in the class $[\rho]$. An explicit important example of nonuniquness for $k=2$ is realized by Helmholtz forms, and will be discussed below in Section 5.1.

- If $q=0$ we define $R_{0}$ be the identity mapping.

Now, by means of the representation mappings $R_{q}$ we construct to the variational sequence $0 \rightarrow \mathbb{R}_{\boldsymbol{Y}} \rightarrow \mathcal{V}_{*}^{r}$ a representation sequence

$$
0 \rightarrow \mathbb{R}_{\boldsymbol{Y}} \rightarrow R_{0}\left(\mathcal{V}_{0}^{r}\right) \rightarrow R_{1}\left(\mathcal{V}_{1}^{r}\right) \rightarrow R_{2}\left(\mathcal{V}_{2}^{r}\right) \rightarrow \cdots
$$

shortly denoted by $0 \rightarrow \mathbb{R}_{\boldsymbol{Y}} \rightarrow R_{*}\left(\mathcal{V}_{*}^{r}\right)$, and called the Takens representation of $0 \rightarrow \mathbb{R}_{\boldsymbol{Y}} \rightarrow \mathcal{V}_{*}^{r}$. We also denote by

$$
E_{q}: R_{q}\left(\mathcal{V}_{q}^{r}\right) \rightarrow R_{q+1}\left(\mathcal{V}_{q+1}^{r}\right), \quad q \geq 1
$$

the morphisms in the representation sequence. Hence, the definition of the $E_{q}$ 's follows from the commutativity of the diagrams

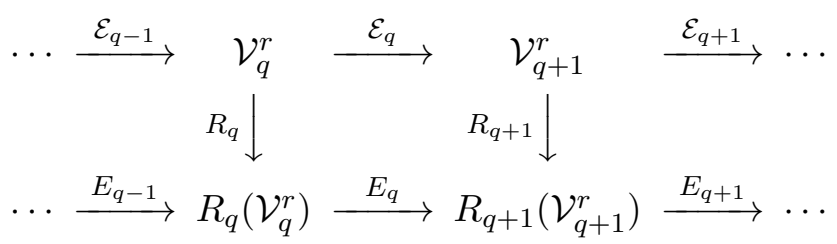

and we can immediately see that the following theorem holds true:

Theorem 3.4. The representation sequence $0 \rightarrow \mathbb{R}_{\boldsymbol{Y}} \rightarrow R_{*}\left(\mathcal{V}_{*}^{r}\right)$ is exact.

Indeed, by definition of $E_{q}$ and $\mathcal{E}_{q}$,

$$
E_{q} \circ R_{q}([\rho])=R_{q+1} \circ \mathcal{E}_{q}([\rho])=R_{q+1}([d \rho]),
$$

and for every $\eta \in R_{q}\left(\mathcal{V}_{q}^{r}\right)$ we have $\eta=R_{q}([\rho])$ for some $[\rho] \in \mathcal{V}_{q}^{r}$. Hence

$$
\begin{aligned}
\left(E_{q+1} \circ E_{q}\right)(\eta) & =\left(E_{q+1} \circ E_{q} \circ R_{q}\right)([\rho])=\left(E_{q+1} \circ R_{q+1} \circ \mathcal{E}_{q}\right)([\rho]) \\
& =\left(E_{q+1} \circ R_{q+1}\right)([d \rho])=\left(R_{q+2} \circ \mathcal{E}_{q+1}([d \rho])=R_{q+2}([d d \rho])=0 .\right.
\end{aligned}
$$

The representation sequence can be easily understood, and explicit formulas for the morphisms $E_{q}$ and the forms $E_{q}(\eta) \in R_{q+1}\left(\mathcal{V}_{q+1}^{r}\right)$ can be easily obtained, if we write the diagram (3.4) in the explicit form

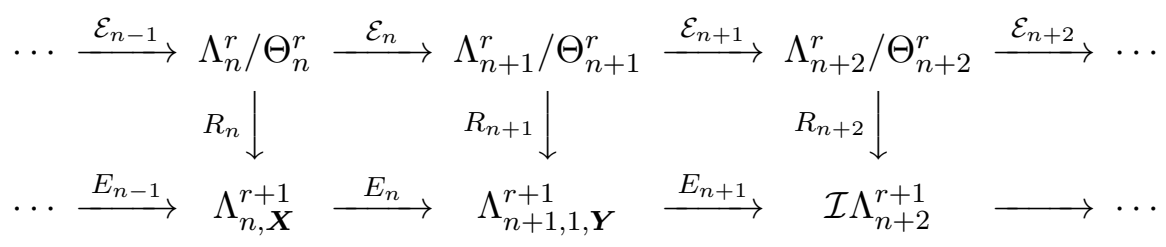

where $R_{q}, 1 \leq q \leq n$, has the meaning of horizontalization $h$ on classes of order $r$, and $R_{n+k}$ is the operator $\mathcal{I}$ acting on $p_{k} \rho$ where $\rho$ is of order $r$. Then since elements of the sheaves $R_{q}\left(\mathcal{V}_{q}^{r}\right)$ are functions for $q=0$, horizontal forms for $1 \leq q \leq n$, and canonical source forms for $q \geq n+1$, we have

$$
\begin{aligned}
& E_{0}(f)=h d f, \quad E_{q}(h \rho)=h d h \rho, \quad 1 \leq q \leq n-1, \\
& E_{n}(h \rho)=\mathcal{I}(d h \rho), \quad E_{n+k}(\mathcal{I}(\rho))=\mathcal{I}(d(\mathcal{I}(\rho))), \quad k \geq 1 .
\end{aligned}
$$


In particular, for $q=n$ we can write in coordinates $h \rho=L \omega_{0}$, where $\omega_{0}=d x^{1} \wedge \cdots \wedge d x^{n}$, and then

$$
E_{n}(h \rho)=R_{n+1}([d \rho])=\mathcal{I}(d \rho)=\mathcal{I}(d h \rho)=\sum_{|J|=0}^{r}(-1)^{|J|} d_{J}\left(\frac{\partial L}{\partial y_{J}^{\sigma}}\right) \omega^{\sigma} \wedge \omega_{0} .
$$

Hence, $E_{n}: R_{n}\left(\mathcal{V}_{n}^{r}\right) \rightarrow R_{n+1}\left(\mathcal{V}_{n+1}^{r}\right)$ is the Euler-Lagrange mapping, assigning to every Lagrangian $\lambda=h \rho$ its Euler-Lagrange form $E_{\lambda}=\mathcal{I}(d \lambda)$. The next morphism, $E_{n+1}: R_{n+1}\left(\mathcal{V}_{n+1}^{r}\right) \rightarrow$ $R_{n+2}\left(\mathcal{V}_{n+2}^{r}\right)$ is the Helmholtz mapping, assigning to every dynamical form $\varepsilon$ its canonical Helmholtz form $H_{\varepsilon}=\mathcal{I}(d \varepsilon)$. Note that the preceding morphism to the Euler-Lagrange morphism, $E_{n-1}: R_{n-1}\left(\mathcal{V}_{n-1}^{r}\right) \rightarrow R_{n}\left(\mathcal{V}_{n}^{r}\right)$, assigns to every horizontal $(n-1)$-form $\varphi$ (resp., if $\operatorname{dim} X=n=1$, to a function $f$ ) a Lagrangian $\lambda=h d \varphi$ (resp. $\lambda=h d f$ ). This is so-called null-Lagrangian (also called variationally trivial Lagrangian), due to the fact that its Euler-Lagrange form is by exactness of the sequence equal to zero.

More generally, for $q \geq 1$, elements of $R_{q}\left(\mathcal{V}_{q}^{r}\right)$ belonging to the kernel of the variational morphism $E_{q}$ are called variationally trivial.

We say that two elements $\eta_{1}, \eta_{2}$ of $R_{q}\left(\mathcal{V}_{q}^{r}\right)$ are (variationally) equivalent if their difference is variationally trivial, i.e., $\eta_{2}-\eta_{1} \in \operatorname{Ker} E_{q}$. Thus, two Lagrangians are equivalent iff they differ by a null Lagrangian, $h d \rho$, two dynamical forms are equivalent iff they differ by a locally variational form, and two Helmholtz-like forms are equivalent iff they differ by a Helmholtz form. Generally, two canonical source forms are equivalent iff they differ by $\mathcal{I}(d(\mathcal{I} \rho))$.

Example 3.5. As an illustration of the use of the Takens representation of the variational sequence let us compute the Euler-Lagrange operator with help of the operator $\mathcal{I}$. Indeed, given a Lagrangian $\lambda$, the Euler-Lagrange form of $\lambda$ arises either as the image of $\lambda$ by the morphism $E_{n}$, or, by application of $\mathcal{I}$ to the exterior derivative of $\lambda$ (remind the formula $E_{n}(\lambda)=\mathcal{I}(d \lambda)$ coming from the commutativity relation $E_{n} \circ \mathcal{I}=\mathcal{I} \circ d$ ).

Consider the Lagrangian of a free quantum particle moving in one dimension. The quantum mechanical state of such a system is, in the coordinate representation, described by a wave function $\phi(t, x): \mathbb{R} \times \mathbb{R} \rightarrow \mathbb{C}$, and the Lagrangian is

$$
\lambda=\left(-\frac{\hbar^{2}}{4 m}\left(\phi_{x} \phi_{x}^{*}\right)+\frac{\mathrm{i} \hbar}{4}\left(\phi^{*} \phi_{t}-\phi \phi_{t}^{*}\right)\right) \omega_{0}, \quad \omega_{0}=d t \wedge d x
$$

Denoting $v(t, x)=\operatorname{Re} \phi(t, x)$ and $w(t, x)=\operatorname{Im} \phi(t, x)$ we obtain

$$
\lambda=\left(-\frac{\hbar^{2}}{4 m}\left(v_{x}^{2}+w_{x}^{2}\right)-\frac{\hbar}{2}\left(v w_{t}-w v_{t}\right)\right) \omega_{0},
$$

which is a first-order Lagrangian for the fibred manifold $\pi: \mathbb{R}^{2} \times \mathbb{R}^{2} \rightarrow \mathbb{R}^{2}$ (hence $n=2, m=2$, $r=1)$ where $\pi$ is the canonical projection, with fibred coordinates $\left(x^{1}, x^{2}, y^{1}, y^{2}, y_{1}^{1}, y_{2}^{1}, y_{1}^{2}, y_{2}^{2}\right)=$ $\left(t, x, v, w, v_{t}, v_{x}, w_{t}, w_{x}\right)$. In this notation, the generating contact 1 -forms on $J^{2}\left(\mathbb{R}^{2} \times \mathbb{R}^{2}\right)$ read as follows: $\omega^{1}=d v-v_{t} d t-v_{x} d x, \omega^{2}=d w-w_{t} d t-w_{x} d x, \omega_{t}^{1}=d v_{t}-v_{t t} d t-v_{t x} d x, \omega_{x}^{1}=$ $d v_{x}-v_{x t} d t-v_{x x} d x, \omega_{t}^{2}=d w_{t}-w_{t t} d t-w_{t x} d x, \omega_{x}^{2}=d w_{x}-w_{x t} d t-w_{x x} d x$. Now, $p_{1} d \lambda$ is the following 1-contact 3 -form on $J^{2}\left(\mathbb{R}^{2} \times \mathbb{R}^{2}\right)$ :

$$
p_{1} d \lambda=\left(-\frac{\hbar}{2} w_{t} d v+\frac{\hbar}{2} v_{t} d w+\frac{\hbar}{2} w d v_{t}-\frac{\hbar}{2} v d w_{t}-\frac{\hbar^{2}}{2 m} v_{x} d v_{x}-\frac{\hbar^{2}}{2 m} w_{x} d w_{x}\right) \omega_{0} .
$$

Calculating $\mathcal{I}(d \lambda)$ using formula (3.1) we obtain

$$
\mathcal{I}(d \lambda)=\omega^{1} \wedge\left[-\frac{\hbar}{2} w_{t}-d_{t}\left(\frac{\hbar}{2} w\right)-d_{x}\left(-\frac{\hbar^{2}}{2 m} v_{x}\right)\right] \omega_{0}
$$




$$
\begin{aligned}
& +\omega^{2} \wedge\left[\frac{\hbar}{2} v_{t}-d_{t}\left(-\frac{\hbar}{2} v\right)-d_{x}\left(-\frac{\hbar^{2}}{2 m} w_{x}\right)\right] \omega_{0} \\
= & {\left[\left(-\hbar w_{t}+\frac{\hbar^{2}}{2 m} v_{x x}\right) \omega^{1}+\left(\hbar v_{t}+\frac{\hbar^{2}}{2 m} w_{x x}\right) \omega^{2}\right] \wedge \omega_{0} . }
\end{aligned}
$$

The dynamical 3-form $\mathcal{I}(d \lambda)$ is the Euler-Lagrange form of (3.5). This means that

$$
\varepsilon_{1}=\frac{\hbar^{2}}{2 m} v_{x x}-\hbar w_{t}, \quad \varepsilon_{2}=\frac{\hbar^{2}}{2 m} w_{x x}+\hbar v_{t},
$$

are the Euler-Lagrange expressions of the Lagrangian (3.5), and equations $\varepsilon_{1} \circ J^{2} \gamma=0$, $\varepsilon_{2} \circ J^{2} \gamma=0$ are the Euler-Lagrange equations for extremals $\gamma$ of (3.5). Denoting $\varepsilon=\varepsilon_{1}+\mathrm{i} \varepsilon_{2}$ we obtain the Euler-Lagrange equation

$$
\left(\frac{\hbar^{2}}{2 m} \phi_{x x}+\mathrm{i} \hbar \phi_{t}\right) \circ J^{2} \gamma=0
$$

which, indeed, is the Schrödinger equation.

\subsection{Lepage $n$-forms and the first variation formula}

Lepage $n$-forms were introduced by Krupka in 1973 [46] (see also [48]) in order to establish foundations of the higher-order calculus of variations in jet bundles. They are fundamental for a geometric formulation of the intrinsic first variation formula, and of coordinate free, global Lagrangian and Hamiltonian mechanics and field theory. Combined with the concepts of invariant variational functionals they provide geometric formulations of Noether theorems, as well as geometric integration methods based on symmetries.

Here we remind only some basic properties of Lepage $n$-forms, explored in the variational sequence theory. For more details and applications we refer the reader to the survey papers $[54,59,60,67,76]$, and the book [55].

As above, $\pi: \boldsymbol{Y} \rightarrow \boldsymbol{X}$ is a fibred manifold, $n=\operatorname{dim} \boldsymbol{X}$, and $m=\operatorname{dim} \boldsymbol{Y}-n$.

Definition 3.6. Let $r \geq 0$. A $n$-form $\rho$ on $J^{r} \boldsymbol{Y}$ is called a Lepage $n$-form of order $r$ if for every $\pi_{r, 0}$-vertical vector field $\xi$ on $J^{r} \boldsymbol{Y}$

$$
h(\xi\rfloor d \rho)=0 .
$$

Since $\rho$ is an $n$-form on $J^{r} \boldsymbol{Y}$ its horizontal component $h \rho$ is a Lagrangian on $J^{r+1} \boldsymbol{Y}$.

Notice that

- every $n$-form on $\boldsymbol{Y}$ is a Lepage $n$-form. The corresponding Lagrangian is then defined on $J^{1} \boldsymbol{Y}$, and it is a polynomial of degree $n$ in the first derivatives ${ }^{1}$,

- every closed $n$-form on $J^{r} \boldsymbol{Y}$ is a Lepage $n$-form. The corresponding Lagrangian is a nullLagrangian (giving rise to the zero Euler-Lagrange form).

The structure of Lepage $n$-forms is characterized as follows:

Theorem 3.7 (Krupka [46]). The following conditions are equivalent:

(1) $\rho$ is a Lepage $n$-form of order $r$,

(2) $p_{1} d \rho$ is a dynamical form, i.e., $p_{1} d \rho=\mathcal{I}(d \rho)$,

\footnotetext{
${ }^{1}$ This fact is of particular importance in Hamiltonian mechanics and field theory, or in dealing with variational forces and energy-momentum tensors [38, 54, 68].
} 
(3) $\pi_{r+1, r}^{*} d \rho=E+F$, where $E$ is a dynamical form, and $F$ is at least 2-contact,

(4) $\pi_{r+1, r}^{*} \rho=\theta_{h \rho}+d \nu+\mu=\theta_{h \rho}+p_{1} d \nu+\eta$, where $\theta_{h \rho}$ is the Cartan form of the Lagrangian $h$, $\nu$ is a contact $(n-1)$-form, and $\mu$, resp. $\eta$ is at least 2 -contact.

It should be stressed that for $n \geq 2$ and $r \geq 3$ the Cartan form is generically not global. However, the above theorem states that it can be "globalized" by adding $p_{1} d \nu$. Indeed, in the decomposition of $\pi_{r+1, r}^{*} \rho$ in (4), $\theta_{h \rho}+p_{1} d \nu$ and $\eta$ are global (being the at most 1-contact and the at least 2-contact part of $\rho$, respectively), while, in general, the decomposition $\theta_{h \rho}+p_{1} d \nu$ is not invariant under changes of fibred coordinates.

The name 'Cartan form' refers to É. Cartan, who introduced the form to the classical calculus of variations [10]. Concerning different aspects of generalization to many independent variables we refer, e.g., to [6, 9, 12, 18, 29, 33, 34, 46, 47, 78, 90]. Higher-order Cartan forms have been introduced and studied by many authors; see, e.g., [12, 13, 21, 22, 30, 40, 48] to name just a few.

Accounting the definition and properties of the operators $\mathcal{I}$ and $\mathcal{R}$ we are able to find an intrinsic formula for the Cartan form:

Theorem 3.8. Let $r \geq 0$, and $\rho \in \Lambda_{n}^{r}$. Then the Cartan form of the Lagrangian $\lambda=h \rho$ takes the form

$$
\theta_{\lambda}=\lambda-p_{1} \mathcal{R}(d \lambda)
$$

Notice that, indeed, $\theta_{h \rho}$ is a Lepage $n$-form, equivalent with $\rho$ (and $h \rho$ ), since by formulas (3.2) and (3.3)

$$
\begin{aligned}
p_{1} d \theta_{h \rho} & =\mathcal{I}\left(d \theta_{h \rho}\right)+p_{1} d p_{1} \mathcal{R}\left(d \theta_{h \rho}\right)=\mathcal{I}\left(d \theta_{h \rho}\right)+p_{1} d p_{1} \mathcal{R}(d h \rho)-p_{1} d p_{1} \mathcal{R}\left(d p_{1} \mathcal{R}(d h \rho)\right) \\
& =\mathcal{I}\left(d \theta_{h \rho}\right)+p_{1} d p_{1} \mathcal{R}(d h \rho)-p_{1} d p_{1} \mathcal{R}(d h \rho)+\mathcal{I}\left(d p_{1} \mathcal{R}(d h \rho)\right)=\mathcal{I}\left(d \theta_{h \rho}\right)
\end{aligned}
$$

and, writing $\pi_{r+1, r}^{*} \rho=h \rho+\beta$ we can see that (for a proper $s$ )

$$
\pi_{s, r}^{*} \rho-\theta_{h \rho}=\beta+p_{1} \mathcal{R}(d h \rho) \in \Theta_{n}^{s} .
$$

The meaning of Lepage $n$-forms for the calculus of variations comes from the fact that if $\rho$ is a Lepage $n$-form then the dynamical form $p_{1} d \rho=E_{h \rho}$ is the Euler-Lagrange form of the Lagrangian $h \rho$. Indeed, the components of $p_{1} d \rho$ are the Euler-Lagrange expressions of the Lagrangian $\lambda=h \rho$.

We stress that even though $\theta_{h \rho}$ need not be global, the local forms do give rise to the global form $p_{1} d \theta_{h \rho}$. Indeed, formula (3.6) yields

$$
p_{1} d \theta_{\lambda}=p_{1} d \lambda-p_{1} d p_{1} \mathcal{R}(d \lambda)=\mathcal{I}(d \lambda)
$$

which, indeed, is a global form. (Note that we recovered the formula for the Euler-Lagrange form of $\lambda$ in Takens representation of the variational sequence.) Moreover, since for every Lepage form $\rho, \pi_{r+1, r}^{*} p_{1} d \rho=p_{1} d \theta_{h \rho}$, we get another proof of uniqueness of the Euler-Lagrange form.

As the horizontal parts of Lepage $n$-forms are Lagrangians, we can view a Lepage $n$-form as an extension of a Lagrangian by a contact form, and we come to the concept of Lepage equivalent of a Lagrangian [46]. Given a Lagrangian $\lambda$, a Lepage equivalent of $\lambda$ is a Lepage form $\rho$ such that $\lambda=h \rho$.

Remarkably, if $\lambda$ is of order $r$, then its Lepage equivalents are generically of order $2 r-1$, and the Euler-Lagrange form is of order $2 r$.

Local existence of Lepage equivalents of $\lambda$ and their structure follows immediately from the above theorem. Moreover, it is well known that every Lagrangian admits a global Lepage equivalent. For $n=1$ and any $r$, or for any $n$ and $r \leq 2$ a global Lepage equivalent of $\lambda$ 
is the Cartan form. If $n=1$ (mechanics and higher-order mechanics) the Cartan form $\theta_{\lambda}$ is the unique Lepage equivalent of $\lambda$. For $n>1$, Lepage equivalent of $\lambda$ is no longer unique. Remarkably, apart from the (globalized) Cartan form, there are also other distinguished global Lepage equivalents of $\lambda$, as, e.g., the celebrated Carathéodory form ([9, 78] for $r=1$, and [92] for $r=2$ ), which is invariant with respect to all (not only fibred) coordinate transformations, and the Krupka-Betounes form (for $r=1$ ) [6, 47], which has the property that $d \rho=0$ if and only if $E_{h \rho}=0$ (i.e., the Lagrangian belongs to Ker $E_{n}$ ).

Example 3.9. Let us illustrate the use of the Lepage representation of the variational sequence on an example. Consider the quantum particle Lagrangian (3.5) in Example 3.5 (including notations). Its Cartan form reads

$$
\begin{aligned}
\theta_{\lambda}= & L \omega_{0}+\frac{\partial L}{\partial v_{t}} \omega^{1} \wedge d x+\frac{\partial L}{\partial w_{t}} \omega^{2} \wedge d x-\frac{\partial L}{\partial v_{x}} \omega^{1} \wedge d t-\frac{\partial L}{\partial w_{x}} \omega^{2} \wedge d t \\
= & \left(-\frac{\hbar^{2}}{4 m}\left(v_{x}^{2}+w_{x}^{2}\right)-\frac{\hbar}{2}\left(v w_{t}-w v_{t}\right)\right) \omega_{0} \\
& +\frac{\hbar^{2}}{2 m} v_{x} \omega^{1} \wedge d t+\frac{\hbar^{2}}{2 m} w_{x} \omega^{2} \wedge d t+\frac{\hbar}{2} w \omega^{1} \wedge d x-\frac{\hbar}{2} v \omega^{2} \wedge d x .
\end{aligned}
$$

As we have seen, being a Lepage equivalent of the Lagrangian (3.5), the form $\theta_{\lambda}$ quickly gives us the Euler-Lagrange form $E_{\lambda}$, and hence the Euler-Lagrange equations:

$$
E_{\lambda}=p_{1} d \theta_{\lambda}=\left(\frac{\hbar^{2}}{2 m} v_{x x}-\hbar w_{t}\right) \omega^{1} \wedge \omega_{0}+\left(\frac{\hbar^{2}}{2 m} w_{x x}+\hbar v_{t}\right) \omega^{2} \wedge \omega_{0} .
$$

We can see that, indeed, $E_{\lambda}$ equals to $\mathcal{I}(d \lambda)$ obtained in Example 3.5; this follows from the commutativity relation $\operatorname{Lep}_{2} \circ E_{2}=d \circ \operatorname{Lep}_{1}$.

We note that the form $\theta_{\lambda}$ (or any other Lepage equivalent of the Lagrangian) also gives local Noetherian conserved currents related with symmetries of the Lagrangian and the EulerLagrange form (see the discussion below).

With help of a Lepage equivalent of a Lagrangian the non-intrinsic procedure of integration by parts in the first variation of the action function is substituted by a geometric splitting with help of the Cartan formula for decomposition of the Lie derivative. As a consequence, the integral first variation formula appears also in the following intrinsic differential form [46] (for more details see, e.g., [54]). Given a Lagrangian $\lambda$ of order $r$, and a $\pi$-projectable vector field $\Xi$ on $\boldsymbol{Y}$ (a "variation"), it holds

$$
\left.\left.L_{J^{r} \Xi} \lambda \equiv L_{J^{2 r} \Xi} h \rho=h\left(J^{2 r-1} \Xi\right\rfloor d \rho\right)+h d\left(J^{2 r-1} \Xi\right\rfloor \rho\right) \equiv h L_{J^{2 r-1} \Xi \rho,}
$$

where $L_{J^{r}} \Xi$ denotes the Lie derivative along the $r$-jet prolongation of $\Xi$, and $\rho$ is (any) Lepage equivalent of $\lambda$. Let us show that the first term in the sum of (3.7) is the Euler-Lagrange term (carrying information about extremals), and the second term is the Noether term, carrying information about conservations laws.

We have $\lambda=h \rho$, and the Euler-Lagrange form is defined by $E_{\lambda}=p_{1} d \varrho$. For the first differential form on the right-hand side of (3.7) we obtain

$$
\left.\left.\left.\left.h\left(J^{2 r-1} \Xi\right\rfloor d \rho\right)=h\left(J^{2 r} \Xi\right\rfloor \pi_{2 r, 2 r-1}^{*} d \rho\right)=h\left(J^{2 r} \Xi\right\rfloor\left(p_{1} d \rho+\mu\right)\right)=h\left(J^{2 r} \Xi\right\rfloor\left(E_{\lambda}\right)\right),
$$

where $\mu$ is at least 2-contact, hence $\left.J^{2 r} \Xi\right\rfloor \mu$ is contact and $\left.h\left(J^{2 r} \Xi\right\rfloor \mu\right)=0$. Denote $E_{\lambda}=$ $E_{\sigma} \omega^{\sigma} \wedge \omega_{0}$ (i.e., $E_{\sigma}$ are the Euler-Lagrange expressions of $\lambda$ ), $\left.\omega_{i}=\frac{\partial}{\partial x^{i}}\right\rfloor \omega_{0}$, and $\Xi=\xi^{i} \frac{\partial}{\partial x^{i}}+\Xi^{\sigma} \frac{\partial}{\partial y^{\sigma}}$. Then

$$
\left.\left.h\left(J^{2 r-1} \Xi\right\rfloor d \rho\right)=h\left(J^{2 r} \Xi\right\rfloor\left(E_{\sigma} \omega^{\sigma} \wedge \omega_{0}\right)\right)=h\left(E_{\sigma} \Xi^{\sigma} \omega_{0}-E_{\sigma} \xi^{i} \omega^{\sigma} \wedge \omega_{i}\right)=E_{\sigma} \Xi^{\sigma} \omega_{0},
$$

showing that, indeed, $\left.h\left(J^{2 r-1} \Xi\right\rfloor d \rho\right)$ provides Euler-Lagrange equations. 
Let us turn to the second differential form in the sum of (3.7). Assume that $\Xi$ is a Noether symmetry of the Lagrangian $\lambda$, meaning that $L_{J^{r} \Xi} \Xi=0$. Then in the infinitesimal first variation formula (3.7) the left-hand side vanishes, and along every extremal $\gamma$ of the Lagrangian $\lambda$ the $n$-form $\left.h\left(J^{2 r-1} \Xi\right\rfloor d \rho\right)$ vanishes, because the Euler-Lagrange equations $E_{\sigma} \circ J^{2 r} \gamma=0,1 \leq \sigma \leq m$, hold true. Consequently, the $n$-form $\left.h d\left(J^{2 r-1} \Xi\right\rfloor \rho\right)$ vanishes along extremals, which is the celebrated Noether theorem. The $(n-1)$-form

$$
\left.\Phi(\Xi)=J^{2 r-1} \Xi\right\rfloor \rho
$$

is then the corresponding Noether current.

In the simplest case of first-order mechanics when $\rho=\theta_{\lambda}=L d t+\frac{\partial L}{\partial \dot{q}^{\sigma}} \omega^{\sigma}$, we recover the well-known formulas

$$
\begin{aligned}
& \left.h\left(J^{1} \Xi\right\rfloor d \theta_{\lambda}\right)=\left(\frac{\partial L}{\partial q^{\sigma}}-\frac{d}{d t} \frac{\partial L}{\partial \dot{q}^{\sigma}}\right) \Xi^{\sigma} d t, \\
& \Phi(\Xi)=L \xi^{0}+\frac{\partial L}{\partial \dot{q}^{\sigma}}\left(\Xi^{\sigma}-\dot{q}^{\sigma} \xi^{0}\right)=-H \xi^{0}+p_{\sigma} \Xi^{\sigma} .
\end{aligned}
$$

If $\Xi$ is a Noether symmetry of $\lambda$, then the function $\Phi(\Xi)$ is constant along extremals.

We shall return to the variational splitting of the Lie derivative in a more general context in Section 4.

\subsection{Lepage forms of higher degrees, and the Lepage representation of the variational sequence}

A motivation for introducing Lepage forms of higher degrees is the following observation: If $\operatorname{dim} X=1$ then every Lagrangian $\lambda$ has a unique Lepage equivalent, the Cartan form $\theta_{\lambda}$. The mapping $\operatorname{Lep}_{1}: \Lambda_{1, \boldsymbol{X}}^{r} \ni \lambda \rightarrow \theta_{\lambda} \in \Lambda_{1}^{2 r-1}$ thus relates the Euler-Lagrange mapping to the exterior derivative through the relationship $E_{\lambda}=p_{1} d \theta_{\lambda}$. This suggests the idea to extend Lep 1 to dynamical forms, i.e., to $\operatorname{Lep}_{2}: \Lambda_{2, \boldsymbol{Y}}^{s} \ni \varepsilon \rightarrow \alpha_{\varepsilon} \in \Lambda_{2}^{s}$, so that we would have

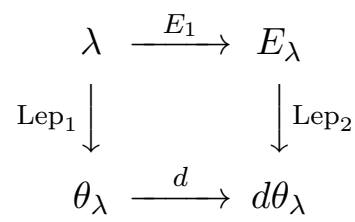

The concept of a Lepage equivalent of a dynamical form was introduced in [64] for locally variational forms. In that situation one obtains an extension of the Euler-Lagrange form to a closed form which (similarly as in the case of a Lepage equivalent of a Lagrangian) is unique for $n=1$ and nonunique otherwise (see [37, 38, 64, 66, 72]). Lepage equivalents of Euler-Lagrange forms became important particularly in study of the inverse variational problem, Hamiltonian theory, and geometric integration based on symmetries of the equations [34, 55, 65, 66, 68, 71].

Having the variational sequence and its Takens representation allows to define the concept of Lepage equivalent for any source form, and, in this way, to link all the variational operators to the exterior derivative. Remarkably, with Lepage forms one gets another representation of the variational sequence where variational morphisms simplify to exterior derivatives. The immediate benefit is the reformulation and solution of the problem of existence of Lagrangians for given differential equations "as they stand", the problem of the structure of null Lagrangians, and the other inverse problems in the variational sequence: by using Lepage forms these problems are reduced to application of the Poincaré lemma.

The idea how to generalize the concept of Lepage form to higher degrees is suggested by property (2) (or (3)) in Theorem 3.7, that the lowest contact component of the exterior derivative of a Lepage form $\rho$ is the canonical source form for $d \rho$ : 
Definition 3.10 ([45,61]). Let $k \geq 0$. A $(n+k)$-form $\rho$ on $J^{r} \boldsymbol{Y}$ is called Lepage form if

$$
p_{k+1} d \rho=\mathcal{I}(d \rho) .
$$

Lepage $(n+k)$-forms for $k>0$ have many similar properties as have Lepage $n$-forms. First,

- every $q$-form on $\boldsymbol{Y}, q \geq n$, is a Lepage form,

- every closed $q$-form on $J^{r} \boldsymbol{Y}, q \geq n$, is a Lepage form.

Structure of Lepage forms can be derived by direct calculations in coordinates from the definition. However, this procedure is quite lengthy and tedious. Much more advantageously, we again explore the operator $\mathcal{R}$ to solve the equation (3.8) with respect to $\rho$ in an intrinsic way. Then we obtain:

Theorem 3.11. Equation (3.8) has the solution

$$
\pi_{r+1, r}^{*} \rho=\theta_{p_{k} \rho}+d \nu+\mu=\theta_{p_{k} \rho}+p_{k+1} d \nu+\eta
$$

where

$$
\theta_{p_{k} \rho}=p_{k} \rho-p_{k+1} \mathcal{R}\left(d p_{k} \rho\right)
$$

$\nu$ is an arbitrary at least $(k+1)$-contact $(n+k-1)$-form, and $\mu($ resp. $\eta)$ is an arbitrary at least $(k+2)$-contact form.

For every choice of $\nu$ and $\mu$ (resp. $\eta$ ) the Lepage forms (3.9) belong to the same variational class $[\rho] \in \mathcal{V}_{n+k}^{s}($ for a proper $s)$, i.e., $\pi_{s, r}^{*} \rho-\theta_{p_{k} \rho} \in \Theta_{n+k}^{s}$.

Proof. First we show that $\theta_{p_{k} \rho}$ given by (3.10) is a Lepage form, i.e., satisfies $p_{k+1} d \theta_{p_{k} \rho}=$ $\mathcal{I}\left(d \theta_{p_{k} \rho}\right)$. Using the same procedure as above for $k=0$, we obtain:

$$
\begin{aligned}
p_{k+1} d \theta_{p_{k} \rho} & =\mathcal{I}\left(d \theta_{p_{k} \rho}\right)+p_{k+1} d p_{k+1} \mathcal{R}\left(d \theta_{p_{k} \rho}\right) \\
& =\mathcal{I}\left(d \theta_{p_{k} \rho}\right)+p_{k+1} d p_{k+1} \mathcal{R}\left(d p_{k} \rho\right)-p_{k+1} d p_{k+1} \mathcal{R}\left(d p_{k+1} \mathcal{R}\left(d p_{k} \rho\right)\right)=\mathcal{I}\left(d \theta_{p_{k} \rho}\right),
\end{aligned}
$$

since

$$
\begin{aligned}
p_{k+1} d p_{k+1} \mathcal{R}\left(d p_{k+1} \mathcal{R}\left(d p_{k} \rho\right)\right) & =p_{k+1} d p_{k+1} \mathcal{R}\left(d p_{k} \rho\right)-\mathcal{I}\left(d p_{k+1} \mathcal{R}\left(d p_{k} \rho\right)\right) \\
& =p_{k+1} d p_{k+1} \mathcal{R}\left(d p_{k} \rho\right),
\end{aligned}
$$

in view of $\mathcal{I}\left(d p_{k+1} \mathcal{R}\left(d p_{k} \rho\right)\right)=\mathcal{I}\left(p_{k+1}\left(d p_{k+1} \mathcal{R}\left(d p_{k} \rho\right)\right)\right)=0$. Hence, $\theta_{p_{k} \rho}$ is a solution of (3.8). Since the equation concerns $d \rho$, it is clear that if $\rho$ is a solution then also $\rho+d \nu$ is a solution, where $\nu$ is an arbitrary $(n+k-1)$-form. Moreover, equation (3.8) allows to determine only the lowest, $(k+1)$-contact component of $d \rho$. Thus, any solution $\rho$ is determined up to $d \nu+\mu$, where $\mu$ is at least $(k+2)$-contact. In this way, so far we have obtained $\rho=\theta_{p_{k} \rho}+d \nu+\mu$, where $\mu$ is at least $(k+2)$-contact. We observe that $p_{k} \theta_{p_{k} \rho}=p_{k} \rho$, hence $p_{k} d \nu$ must be equal to zero, meaning that $\nu$ is at least $(k+1)$-contact.

Finally, we have to show that whatever the choice of $\nu$ and $\mu, \rho$ is equivalent with $\theta_{p_{k} \rho}$. This is, however, easily seen: if we denote by $s$ the order of $\theta_{p_{k} \rho}$, and by $\beta$ the at least $(k+1)$-contact component of $\rho$, we obtain $\pi_{s, r}^{*} \rho-\theta_{p_{k} \rho}=p_{k} \rho+\beta-\theta_{p_{k} \rho}=\beta+p_{k+1} \mathcal{R}\left(d p_{k} \rho\right)$, which is a strongly contact $(n+k)$-form, thus belonging to the kernel $\Theta_{n+k}^{s}$.

The $(n+k)$-form $\theta_{p_{k} \rho}$ is the higher-degree generalization of the Cartan form. It is completely determined by its lowest contact ( $k$-contact) component. Similarly as the Cartan form of degree $n$ it is generically not global, since it is defined by means of the operator $\mathcal{R}$. However, quite similarly as in the case of Lepage $n$-forms, it can be "globalized" by adding a proper term $p_{k+1} d \nu$. 
And similarly as in the familiar $k=0$ situation, $p_{k+1} d \theta_{p_{k} \rho}$ is global, and it holds

$$
p_{k+1} d \theta_{p_{k} \rho}=\mathcal{I}\left(d p_{k} \rho\right) .
$$

Also in the higher degree situation, there is a distinguished case of $n=1$ (and arbitrary $r$ ) (mechanics). Then $\nu$ is a $k$-form and $\mu$ is a $(k+1)$-form, hence they both are zero and we have:

Corollary 3.12. Let $n=\operatorname{dim} \boldsymbol{X}=1, r \geq 0$. Then for every $k \geq 0$, the higher degree Cartan form $\theta_{p_{k} \rho}$ is unique and hence global.

The higher-degree generalization of Theorem 3.7 now reads as follows:

Theorem 3.13. Let $k \geq 0$. The following conditions are equivalent:

(1) $\rho$ is a Lepage $(n+k)$-form of order $r$,

(2) $\pi_{r+1, r}^{*} d \rho=\mathcal{I}(d \rho)+F$, where $F$ is at least $(k+2)$-contact,

(3) $p_{k+1} d \mathcal{R}\left(p_{k+1} d \rho\right)=0$,

(4) $\pi_{r+1, r}^{*} \rho=\theta_{p_{k} \rho}+d \nu+\mu=\theta_{p_{k} \rho}+p_{k+1} d \nu+\eta$, where $\theta_{p_{k} \rho}=p_{k} \rho-p_{k+1} \mathcal{R}\left(d p_{k} \rho\right)$, $\nu$ is an at least $(k+1)$-contact $(n+k-1)$-form, and $\mu$, resp. $\eta$ is at least $(k+2)$-contact.

Again, in the decomposition of $\pi_{r+1, r}^{*} \rho$ in (4), $\theta_{p_{k} \rho}+p_{k+1} d \nu$ and $\eta$ are global (being the at most $(k+1)$-contact and the at least $(k+2)$-contact part of $\rho$, respectively), while, in general, the decomposition $\theta_{p_{k} \rho}+p_{k+1} d \nu$ is not invariant under changes of fibred coordinates. And $p_{k+1} d \rho$ is unique (independent upon a choice of $\rho$ ).

Also the concept of Lepage equivalent of a Lagrangian extends to $(n+k)$-forms. Given a $k$-contact form $\sigma$, by a Lepage equivalent of $\sigma$ we mean a Lepage form $\rho$ such that $p_{k} \rho=\sigma$. Theorem 3.13 then gives the structure of Lepage equivalents, and guarantees local existence.

There remains to answer a question about existence of global Lepage equivalents of higher degrees. The result is affirmative, and its proof is a generalization of a proof for $n$-forms [48]. The idea is to show that there exists a Lepage equivalent which is a global form on a closed submanifold of $J^{2 r+1} \boldsymbol{Y}$. Then, since the (local) form defines a soft sheaf of forms it can be extended to a global form on the whole space.

Let us consider the canonical injection $\iota_{r+1, r}: J^{2 r+1} \boldsymbol{Y} \rightarrow J^{r+1}\left(J^{r} \boldsymbol{Y}\right)$ defined by $\iota_{r+1, r}\left(j_{x}^{2 r+1} \gamma\right)$ $=j_{x}^{r+1}\left(J^{r} \gamma\right)$.

Theorem 3.14. Let $\tilde{\phi}$ be a $(n+k)$-form such that $p_{l} \tilde{\phi}=0, l \geq k+1$, e.g., $\tilde{\phi}$ is the $p_{k}$ component defined on $J^{1}\left(J^{r} \boldsymbol{Y}\right)$ of a $(n+k)$-form on $J^{r} \boldsymbol{Y}$. There exists a $(n+k)$-form $\tilde{\eta}$ on $J^{r+1}\left(J^{r} \boldsymbol{Y}\right)$ such that

(1) $\tilde{\eta}$ is $\left(\pi_{r}\right)_{r+1,0}$-horizontal and $\tilde{\eta}$ is a $(k+1)$-contact $(n+k)$-form on $J^{r+1}\left(J^{r} \boldsymbol{Y}\right)$;

(2) for every $\left(\pi_{r}\right)_{r+1,0}$-vertical vector field $\boldsymbol{Z}$ on $J^{r+1}\left(J^{r} \boldsymbol{Y}\right)$ (i.e., such that the projection of $\boldsymbol{Z}$ on $J^{r} \boldsymbol{Y}$ is zero)

$$
\iota_{r+1, r}^{*} L_{\boldsymbol{Z}}\left(\left(\pi_{r}\right)_{r+1,1}^{*} \tilde{\phi}+\tilde{\eta}\right)
$$

is a $(k+1)$-contact $(n+k)$-form on $J^{r+1}\left(J^{r} \boldsymbol{Y}\right)$;

(3) the form $\iota_{r+1, r}^{*}\left(\left(\pi_{r}\right)_{r+1,1}^{*} \tilde{\phi}+\tilde{\eta}\right)$ is a Lepage $(n+k)$-form.

Proof. It is enough to prove uniqueness of $\tilde{\eta}$ when restricted to the submanifold $\iota_{r+1, r}\left(J^{2 r+1} \boldsymbol{Y}\right)$. In fact, for $\rho$ a $(n+k)$ form on $J^{r} \boldsymbol{Y}$, we can take $\tilde{\eta}=-\mathcal{I}\left(p_{k} \rho\right)$ when $\tilde{\phi}=p_{k} \rho$. The result follows by the uniqueness of $\mathcal{I}\left(p_{k} \rho\right)$ which implies that it is a globally defined form on a closed subset of $J^{2 r+1} \boldsymbol{Y}$ (defined by the injection above), therefore since the (local) form defines a soft sheaf of forms on $J^{2 r+1} \boldsymbol{Y}$, then $\tilde{\eta}$ can be globalized. 
The most important Lepage forms of degree $>n$ are Lepage equivalents of canonical source forms. By the above, if $\sigma$ is a canonical source form of degree $n+k, k \geq 1$, and order $r$, i.e., $\mathcal{I}(\sigma)$ is $\pi_{2 r+1, r}$-projectable, and $p_{k} \sigma=\sigma=\mathcal{I}(\sigma)$, then all its local Lepage equivalents take the form

$$
\rho=\theta_{\sigma}+d \nu+\mu=\theta_{\sigma}+p_{k+1} d \nu+\eta,
$$

where

$$
\theta_{\sigma}=\sigma-p_{k+1} \mathcal{R}(d \sigma),
$$

$\nu$ is an arbitrary at least $(k+1)$-contact $(n+k-1)$-form, and $\mu$ (resp. $\eta)$ is an arbitrary at least $(k+2)$-contact form. Moreover,

$$
p_{k+1} d \theta_{\sigma}=\mathcal{I}(d \sigma) \text {. }
$$

In particular, if $\varepsilon$ is a dynamical form then $p_{2} d \theta_{\varepsilon}$ is the canonical Helmholtz form $H_{\varepsilon}=\mathcal{I}(d \varepsilon)$.

Comparing Lepage forms of different degrees yields the following results:

Theorem 3.15. Let $q \geq n$. If $\alpha$ is a Lepage equivalent of $R_{q}([\rho])$ then $d \alpha$ is a Lepage equivalent of $R_{q+1}([d \rho])$.

Proof. Since $\alpha$ is a Lepage form, $d \alpha$ is Lepage, as trivially follows from the definition. Denote $\sigma=R_{q}([\rho])$. If $q=n, \sigma$ is a Lagrangian, hence $R_{n}([\rho])=h \rho$, and $R_{n+1}([d \rho])=\mathcal{I}(d \rho)=$ $\mathcal{I}(d h \rho)$. Thus $\alpha=\theta_{h \rho}+d \nu+\mu$, and $d \alpha$ is a Lepage equivalent of $p_{1} d \alpha$. However, by (3.12), $p_{1} d \alpha=p_{1} d \theta_{h \rho}=\mathcal{I}(d h \rho)=R_{n+1}([d \rho])$. If $q=n+k, k>1$, we have $R_{n+k}([\rho])=\mathcal{I}(\rho)$, and $R_{n+k+1}([d \rho])=\mathcal{I}(d \rho)=\mathcal{I}(d \mathcal{I}(\rho))=\mathcal{I}(d \sigma)$. The form $d \alpha$ is a Lepage equivalent of $p_{k+1} d \alpha$, which by (3.12) is equal to $p_{k+1} d \theta_{\sigma}=\mathcal{I}(d \sigma)=R_{n+k+1}([d \rho])$.

Theorem 3.16. A closed Lepage equivalent of a canonical source form of degree $n+k, k \geq 1$, is locally equal to d $\alpha$ where $\alpha$ is a Lepage equivalent of a canonical source form of degree $n+k-1$ if $k \geq 2$, resp. of a Lagrangian if $k=1$.

Proof. If $\beta$ is a closed Lepage equivalent of $\sigma=p_{k} \beta=\mathcal{I}(\beta)$, then locally $\beta=d \alpha$ where (with help of the contact homotopy operator), $\alpha=\mathcal{A} \beta$, and $p_{k-1} \alpha=\mathcal{A} p_{k} \beta=\mathcal{A} \sigma$.

If $k=1$ then $p_{0} \alpha=h \alpha$ is a Lagrangian, and $p_{1} d \alpha=p_{1} \beta=\mathcal{I}(\beta)$, so that $\alpha=\mathcal{A} \beta$ is a Lepage equivalent of $h \alpha=\mathcal{A} \sigma$.

If $k \geq 2$, set $\varepsilon=\mathcal{I}(\mathcal{A} \sigma)$. $\varepsilon$ is a canonical source form of degree $n+k-1$ representing the class $[\mathcal{A} \sigma]$. Therefore (by the preceding theorem), if $\alpha^{\prime}$ is a Lepage equivalent of $\mathcal{I}(\mathcal{A} \sigma)$ then $d \alpha^{\prime}$ is a Lepage equivalent of $\mathcal{I}(d \varepsilon)=\mathcal{I}(d \mathcal{A} \sigma)=\mathcal{I}\left(d p_{k-1} \alpha\right)=\mathcal{I}(d \alpha)=\mathcal{I}(\beta)=\sigma$. Now, both $d \alpha^{\prime}$ and $\beta$ are Lepage equivalents of $\sigma$, hence we have $\beta=d \alpha^{\prime}+d \nu+\mu$, where $\nu$ is at least $(k+1)$ contact and $\mu$ is at least $(k+2)$-contact, and since $d \beta=0$, we get $d \mu=0$, i.e., locally $\mu=d \tau$ and $\tau=\mathcal{A} \mu$. Hence, $\beta=d\left(\alpha^{\prime}+\nu+\tau\right)$, and $\alpha=\mathcal{A} \beta=\alpha^{\prime}+\nu+\tau$ is a Lepage form (differing from the Lepage $(k-1)$-form $\alpha^{\prime}$ by an at least $(k+1)$-contact form), and it is a Lepage equivalent of $p_{k-1} \alpha=p_{k-1} \alpha^{\prime}=\mathcal{I}(\mathcal{A} \sigma)$, as required. Moreover, we note that since $p_{k-1} \alpha=\mathcal{A} p_{k} \beta=\mathcal{A} \sigma$, it holds $\mathcal{I}(\mathcal{A} \sigma)=\mathcal{A} \sigma=\mathcal{A}(\mathcal{I} \sigma)$.

\section{Corollary 3.17.}

(1) For every canonical source form $\sigma$

$$
\mathcal{I}(\mathcal{A} \sigma)=\mathcal{A} \sigma=\mathcal{A}(\mathcal{I} \sigma) .
$$

(2) If $\sigma$ is a canonical source $(n+k)$-form then $\mathcal{A} \sigma$ is a canonical source $(n+k-1)$-form. 


\section{Corollary 3.18.}

(1) If $\beta$ is a closed Lepage equivalent of a dynamical form $\varepsilon$ then $\mathcal{A} \beta$ is a Lepage equivalent of the Lagrangian $\lambda=\mathcal{A} \varepsilon$.

(2) If $\beta$ is a closed Lepage equivalent of a canonical source form $\sigma$ then $\mathcal{A} \beta$ is a Lepage equivalent of the canonical source form $\mathcal{A} \sigma$.

The above properties of Lepage forms suggest a new representation of the variational sequence, based on Lepage equivalents of canonical source forms, as follows: For every $k \geq 0$ we have a mapping Lep $\operatorname{Len}_{n+k}$ assigning to every Lagrangian $\lambda \in \Lambda_{n, \boldsymbol{X}}^{r}$, if $k=0$, and every canonical source form $\sigma \in \mathcal{I}\left(\Lambda_{n+k}^{r}\right)$ if $k \geq 1$, the family $\{\rho\}$ of its Lepage equivalents (3.11).

With help of Lepage mappings Theorem 3.15 can be reformulated as follows:

Theorem 3.15'. If $\operatorname{Lep}_{q}\left(R_{q}([\rho])\right)=\{\alpha\}$ then $\operatorname{Lep}_{q+1}\left(R_{q+1}([d \rho])\right)=\{d \alpha\}$, or

$$
\left\{d \operatorname{Lep}_{q}\left(R_{q}([\rho])\right)\right\}=\left\{\operatorname{Lep}_{q+1}\left(R_{q+1}([d \rho])\right)\right\} .
$$

The Lepage maps then induce the following representation mappings:

$$
\tilde{R}_{q}=R_{q} \quad \text { for } \quad 0 \leq q \leq n-1 \quad \text { and } \quad \tilde{R}_{q}=\operatorname{Lep}_{q} \circ R_{q} \quad \text { for } \quad q \geq n .
$$

Note that for $q>n$ the images by $\tilde{R}_{q}$ of classes in $\mathcal{V}_{q}^{r}$ are subsheaves of $\Lambda_{q}^{r}$.

With help of the representation mappings $\tilde{R}_{q}$ we construct to the variational sequence $0 \rightarrow$ $\mathbb{R}_{\boldsymbol{Y}} \rightarrow \mathcal{V}_{*}^{r}$ a representation sequence

$$
0 \rightarrow \mathbb{R}_{\boldsymbol{Y}} \rightarrow \tilde{R}_{0}\left(\mathcal{V}_{0}^{r}\right) \rightarrow \tilde{R}_{1}\left(\mathcal{V}_{1}^{r}\right) \rightarrow \tilde{R}_{2}\left(\mathcal{V}_{2}^{r}\right) \rightarrow \cdots
$$

shortly denoted by $0 \rightarrow \mathbb{R}_{\boldsymbol{Y}} \rightarrow \tilde{R}_{*}\left(\mathcal{V}_{*}^{r}\right)$, and called the Lepage representation of the variational sequence $0 \rightarrow \mathbb{R}_{\boldsymbol{Y}} \rightarrow \mathcal{V}_{*}^{r}$.

Theorem 3.19. The representation sequence $0 \rightarrow \mathbb{R}_{\boldsymbol{Y}} \rightarrow \tilde{R}_{*}\left(\mathcal{V}_{*}^{r}\right)$ is exact, and every morphism $\tilde{R}_{q}\left(\mathcal{V}_{q}^{r}\right) \rightarrow \tilde{R}_{q+1}\left(\mathcal{V}_{q+1}^{r}\right)$ for $q \geq n$, is the exterior derivative operator d (acting on classes of Lepage forms).

Proof. Denote

$$
\tilde{E}_{q}: \tilde{R}_{q}\left(\mathcal{V}_{q}^{r}\right) \rightarrow \tilde{R}_{q+1}\left(\mathcal{V}_{q+1}^{r}\right), \quad q \geq n .
$$

With the representation mapping $\tilde{R}_{q}$, Theorem 3.15 states that 'if $\tilde{R}_{q}([\rho])=\{\alpha\}$ then $\tilde{R}_{q+1}([d \rho])$ $=\{d \alpha\}^{\prime}$, in other words, the exterior derivative operator $d$ extends in an obvious way to classes of Lepage forms; denoting the exterior derivative of classes by the same symbol $d$, we can see that

$$
\tilde{E}_{q}(\{\alpha\})=\{d \alpha\} \equiv d\{\alpha\} .
$$

This proves that $\tilde{E}_{q}=d, q \geq n$.

In view of this result, and since $\tilde{R}_{q}=R_{q}$ for $0 \leq q \leq n-1$, and the Takens representation sequence is exact, it remains to check the exactness in the arrow $E_{n-1}$. To this end, let us write the variational sequence and its Lepage representation in form of the following diagram:

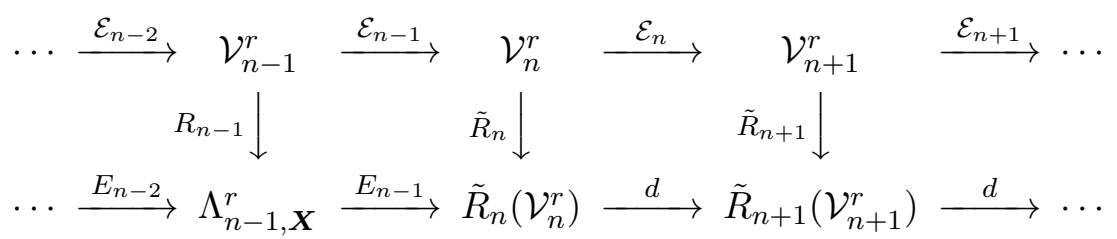


Then for every $\eta \in \tilde{R}_{n-1}\left(\mathcal{V}_{n-1}^{r}\right)=\Lambda_{n-1, \boldsymbol{X}}^{r}$ we obtain

$$
\begin{aligned}
\left(d \circ E_{n-1}\right)(\eta) & =\left(d \circ E_{n-1}\right)\left(R_{n-1}([\rho])\right)=\left(d \circ E_{n-1} \circ R_{n-1}\right)([\rho]) \\
& =\left(d \circ \tilde{R}_{n} \circ \mathcal{E}_{n-1}\right)([\rho])=d\left(\tilde{R}_{n}([d \rho])\right)=d \operatorname{Lep}_{n}\left(R_{n}([d \rho])\right. \\
& =\operatorname{Lep}_{n+1}\left(R_{n+1}([d d \rho])=0 .\right.
\end{aligned}
$$

It is worth to write all the three sequences within one scheme as follows:

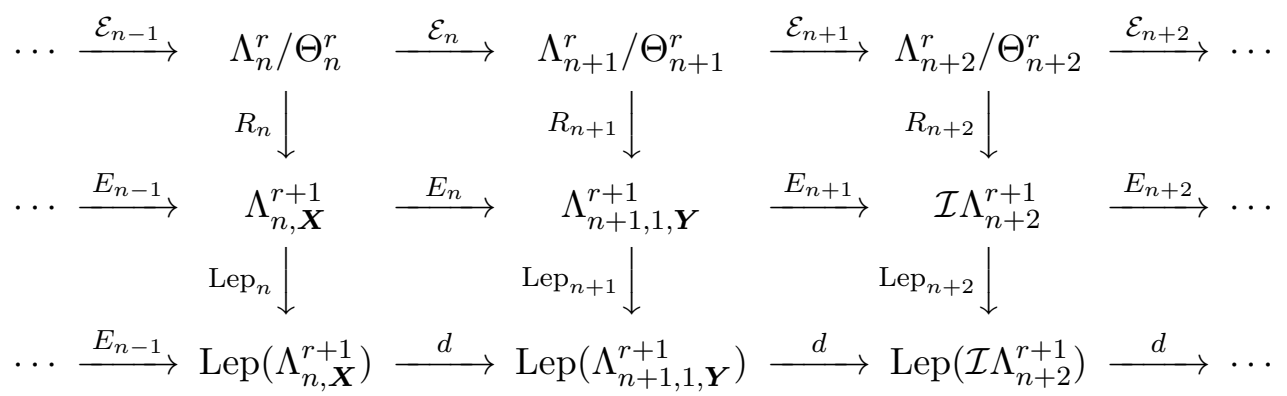

As we have seen, for $n=\operatorname{dim} X=1$ (mechanics), Lepage equivalent is unique for every $k \geq 0$ (recall that this is the Cartan form of degree $n+k$ ). This means that for every $k$,

$$
\operatorname{Lep}_{n+k}: \mathcal{I}\left(\Lambda_{n+k}^{*}\right) \rightarrow \Lambda_{n+k}^{*},
$$

i.e., $\operatorname{Lep}_{n+k}(\mathcal{I} \rho)$ is an element of the sheaf $\Lambda_{n+k}^{*}$ (rather than a family of elements of the sheaf), and $d$ is the "true" exterior derivative of differential forms. Hence "morally" (up to orders of the sheaves) the Lepage representation sequence becomes a subsequence of the de Rham sequence.

Finally we want to stress that in view of the two corollaries of Theorem 3.15 the contact homotopy operator $\mathcal{A}$ restricts to canonical source forms. This means that one can obtain primitives (inverse images) not only of closed Lepage forms in the Lepage representation, but also of canonical source forms in the Takens representation. For a primitive of a variational dynamical form $\varepsilon$ (i.e., a Lagrangian) one has $\lambda=\mathcal{A} \varepsilon$ which in coordinates is the celebrated Tonti Lagrangian [94, 95]. An analogous formula holds then, indeed, also for forms of higher degrees: if $\sigma=E_{n+k}(\eta)$ then $\mathcal{A} \sigma=\eta$.

Example 3.20. Let us write down explicit examples of Cartan forms of higher degrees in mechanics $(n=1)$. Denote local fibred coordinates on $\boldsymbol{Y}$ by $\left(t, q^{\sigma}\right)$ and the associated coordinates on $J^{3} \boldsymbol{Y}$ by $\left(t, q^{\sigma}, \dot{q}^{\sigma}, \ddot{q}^{\sigma}, \dddot{q}^{\sigma}\right)$, and let

$$
\omega^{\sigma}=d q^{\sigma}-\dot{q}^{\sigma} d t, \quad \dot{\omega}^{\sigma}=\omega_{1}^{\sigma}=d \dot{q}^{\sigma}-\ddot{q}^{\sigma} d t, \quad \ddot{\omega}^{\sigma}=\omega_{2}^{\sigma}=d \ddot{q}^{\sigma}-\dddot{q}^{\sigma} d t .
$$

By Theorem 3.11, formula (3.10) we have:

- For $\rho$ of degree $n=1$ and such that $h \rho=\lambda=L d t$ is of order $r=1: \mathcal{R}(d \lambda)=-\frac{\partial L}{\partial \dot{q}^{\sigma}} \omega^{\sigma}$, hence we obtain

$$
\theta_{\lambda}=\lambda+\frac{\partial L}{\partial \dot{q}^{\sigma}} \omega^{\sigma}
$$

which is the classical Cartan 1-form.

- For $\rho$ of degree $n+1=2$ such that $p_{1} \rho$ is a second-order dynamical form, i.e., $p_{1} \rho=\varepsilon=$ $E_{\sigma} \omega^{\sigma} \wedge d t$, the Cartan 2-form is $\theta_{\varepsilon}=\varepsilon-\mathcal{R}(d \varepsilon)$. To compute $\mathcal{R}(d \varepsilon)$ we need the following formula for the residual operator applied to a second-order 2-contact 3-form: denote

$$
\alpha=\sum_{i, j=0}^{2} A_{\sigma \nu}^{i j} \omega_{i}^{\sigma} \wedge \omega_{j}^{\nu} \wedge d t, \quad A_{\sigma \nu}^{i j}=-A_{\nu \sigma}^{j i}
$$


Then

$$
\begin{aligned}
\mathcal{R}(\alpha)= & \frac{1}{2}\left(A_{\sigma \nu}^{1 j}-A_{\nu \sigma}^{j 1}-\frac{d}{d t}\left(A_{\sigma \nu}^{2 j}-A_{\nu \sigma}^{j 2}\right)\right) \omega^{\sigma} \wedge \omega_{j}^{\nu} \\
& -\frac{1}{2}\left(A_{\sigma \nu}^{2 j}-A_{\nu \sigma}^{j 2}\right) \omega^{\sigma} \wedge \omega_{j+1}^{\nu}+\frac{1}{2}\left(A_{\sigma \nu}^{2 j}-A_{\nu \sigma}^{j 2}\right) \omega_{1}^{\sigma} \wedge \omega_{j}^{\nu}
\end{aligned}
$$

(summation over $j=0,1,2$ ). Taking $\alpha=d \varepsilon$ we obtain

$$
\theta_{\varepsilon}=\varepsilon+\frac{1}{2}\left(\frac{\partial E_{\sigma}}{\partial \dot{q}^{\nu}}-\frac{d}{d t} \frac{\partial E_{\sigma}}{\partial \ddot{q}^{\nu}}\right) \omega^{\sigma} \wedge \omega^{\nu}-\frac{\partial E_{\sigma}}{\partial \ddot{q}^{\nu}} \dot{\omega}^{\sigma} \wedge \omega^{\nu}
$$

For a locally variational form $\varepsilon$ this is the Lepage equivalent of $\varepsilon$, introduced in [64] (see also [65]).

- For $\rho$ of degree $n+2=3$ such that $p_{2} \rho$ is a Helmholtz-like form denote $p_{2} \rho=\eta=$ $H_{\sigma \nu}^{0} \omega^{\sigma} \wedge \omega^{\nu} \wedge d t+H_{\sigma \nu}^{1} \omega^{\sigma} \wedge \dot{\omega}^{\nu} \wedge d t+H_{\sigma \nu}^{2} \omega^{\sigma} \wedge \ddot{\omega}^{\nu} \wedge d t$; the Cartan 3-form is $\theta_{\eta}=\eta-\mathcal{R}(d \eta)$. The formula for the residual operator applied to a general second-order 3 -contact 4 -form

$$
\alpha=\sum_{i, j, k=0}^{2} A_{\sigma \nu \rho}^{i j k} \omega_{i}^{\sigma} \wedge \omega_{j}^{\nu} \wedge \omega_{k}^{\rho} \wedge d t, \quad A_{\sigma \nu \rho}^{i j k}=-A_{\nu \sigma \rho}^{j i k}=A_{\nu \rho \sigma}^{j k i},
$$

reads

$$
\begin{aligned}
\mathcal{R}(\alpha)= & -\left(\left\{A_{\sigma \nu \rho}^{1 j k}\right\}-\frac{d}{d t}\left\{A_{\sigma \nu \rho}^{2 j k}\right\}\right) \omega^{\sigma} \wedge \omega_{j}^{\nu} \wedge \omega_{k}^{\rho}+2\left\{A_{\sigma \nu \rho}^{2 j k}\right\} \omega^{\sigma} \wedge \omega_{j}^{\nu} \wedge \omega_{k+1}^{\rho} \\
& -\left\{A_{\sigma \nu \rho}^{2 j k}\right\} \omega_{1}^{\sigma} \wedge \omega_{j}^{\nu} \wedge \omega_{k}^{\rho},
\end{aligned}
$$

where $\left\{A_{\sigma \nu \rho}^{i j k}\right\}=\frac{1}{3}\left(A_{\sigma \nu \rho}^{i j k}+A_{\rho \sigma \nu}^{k i j}+A_{\nu \rho \sigma}^{j k i}\right)$, and summation over $j, k=0,1,2$ applies. Now we take $\alpha=d \eta$, so that

$$
\begin{aligned}
& \left\{A_{\sigma \nu \rho}^{000}\right\}=\left\{\frac{\partial H_{\sigma \nu}^{0}}{\partial q^{\rho}}\right\}, \quad\left\{A_{\sigma \nu \rho}^{001}\right\}=\left\{\frac{\partial H_{\sigma \nu}^{0}}{\partial \dot{q}^{\rho}}+\frac{\partial H_{\nu \rho}^{1}}{\partial q^{\sigma}}\right\}, \quad\left\{A_{\sigma \nu \rho}^{011}\right\}=\left\{\frac{\partial H_{\sigma \nu}^{1}}{\partial \dot{q}^{\rho}}\right\}, \\
& \left\{A_{\sigma \nu \rho}^{002}\right\}=\left\{\frac{\partial H_{\sigma \nu}^{0}}{\partial \ddot{q}^{\rho}}+\frac{\partial H_{\nu \rho}^{2}}{\partial q^{\sigma}}\right\}, \quad\left\{A_{\sigma \nu \rho}^{012}\right\}=\left\{\frac{\partial H_{\sigma \nu}^{1}}{\partial \ddot{q}^{\rho}}-\frac{\partial H_{\sigma \rho}^{2}}{\partial \dot{q}^{\nu}}\right\}, \quad\left\{A_{\sigma \nu \rho}^{022}\right\}=\left\{\frac{\partial H_{\sigma \nu}^{2}}{\partial \ddot{q}^{\rho}}\right\},
\end{aligned}
$$

and $\left\{A_{\sigma \nu \rho}^{i j k}\right\}=0$ for $i, j, k \neq 0$, and obtain the Cartan 3-form of $\eta$ as follows:

$$
\begin{aligned}
\theta_{\eta}= & \eta-\mathcal{R}(d \eta)=\eta+\left(\left\{A_{\sigma \nu \rho}^{100}\right\}-\frac{d}{d t}\left\{A_{\sigma \nu \rho}^{200}\right\}\right) \omega^{\sigma} \wedge \omega^{\nu} \wedge \omega^{\rho} \\
& +2\left(\left\{A_{\sigma \nu \rho}^{101}\right\}-\left\{A_{\sigma \nu \rho}^{200}\right\}+\frac{1}{2}\left\{A_{\sigma \nu \rho}^{002}\right\}-\frac{d}{d t}\left\{A_{\sigma \nu \rho}^{201}\right\}\right) \omega^{\sigma} \wedge \omega^{\nu} \wedge \dot{\omega}^{\rho} \\
& +2\left(\left\{A_{\sigma \nu \rho}^{102}\right\}-\left\{A_{\sigma \nu \rho}^{201}\right\}-\frac{d}{d t}\left\{A_{\sigma \nu \rho}^{202}\right\}\right) \omega^{\sigma} \wedge \omega^{\nu} \wedge \ddot{\omega}^{\rho}-2\left\{A_{\sigma \nu \rho}^{202}\right\} \omega^{\sigma} \wedge \omega^{\nu} \wedge \dddot{\omega}^{\rho} \\
& +2\left(\left\{A_{\sigma \nu \rho}^{021}\right\}-\left\{A_{\sigma \nu \rho}^{210}\right\}\right) \omega^{\sigma} \wedge \dot{\omega}^{\nu} \wedge \dot{\omega}^{\rho}+2\left(\left\{A_{\sigma \nu \rho}^{022}\right\}-\left\{A_{\sigma \nu \rho}^{202}\right\}\right) \omega^{\sigma} \wedge \dot{\omega}^{\nu} \wedge \ddot{\omega}^{\rho}
\end{aligned}
$$

with the non-zero A's as above (cf. [69] where a corresponding formula for the variationally trivial case was obtained).

\subsection{The variational order}

A key issue of the Krupka variational sequence for the variational calculus, making the main difference between his and other approaches $(C$-spectral sequences, the variational bicomplex and infinite order variational sequences) is that the variational sequence (2.2) fixes (and thus defines) the order of variational problems in the sense of the following definition: 
Definition 3.21. Let $\eta \in \Lambda_{q}^{s}$ be a source form, or a Lepage form of degree $q \geq 1$ defined on (an open subset of) $J^{s} \boldsymbol{Y}$. We say that $\eta$ has the variational order $r$ if it comes from the variational sequence of order $r$ (i.e., it is a Takens or Lepage representation of a variational class $[\rho] \in \Lambda_{q}^{r} / \Theta_{q}^{r}$.

How to understand this concept? A variational class $[\rho] \in \Lambda_{q}^{r} / \Theta_{q}^{r}$ concerns local differential forms of degree $q$ and order $r$. Takens representation (and thus also Lepage representation) is based on the horizontalization operator $h=p_{0}$ and the interior Euler operator $\mathcal{I}$ which provide a differential form of generally higher order. For example, if $r=1$ we have (for $q=n$ ) the Lagrangian $\lambda=R_{n}([\rho])=h \rho$, (for $\left.q=n+1\right)$ the dynamical form $\varepsilon=R_{n+1}([\rho])=\mathcal{I}(\rho)$, (for $q=n+2)$ the Helmholtz-like form $H=R_{n+2}([\rho])=\mathcal{I}(\rho)$, etc., all of order 2. As given by the structure of $\mathcal{I}$, generically, the components of the differential $(n+k)$-forms $(k \geq 1)$ arising from $\Lambda_{n+k}^{r} / \Theta_{n+k}^{r}$ are polynomials in the derivatives starting from the order $r+1$ up to the order $2 r$ in the linear term. Notice that such a polynomial behaviour is known to be typical for functions known as "variational derivatives". However the point is that the "variational derivatives" are not just general polynomials: the analysis of $\mathcal{I}$ shows that the coefficients of these polynomials have a determined structure of derivatives of the components of $\rho$ (which are of order $r$ ), with prescribed symmetrization/skew-symmetrization rules in the indices, in other words they have to satisfy certain symmetry and integrability conditions ("order reducibility conditions"). For instance, a dynamical form $\varepsilon$ of order 2 (which defines a system of second order differential equations) ${ }^{2}$ can be of variational order 1 or 2 . In particular, if $\varepsilon$ is locally variational (comes locally from a Lagrangian, i.e., its image by the Helmholtz morphism in the Takens representation sequence vanishes) we obtain that if the variational order of $\varepsilon$ is 1 , we have local Lagrangians arising as $\lambda=h \rho$ from first order $\rho$; thus $\lambda$ is of order 2 , or of order 1 if $h \rho$ is $\pi_{2,1}$-projectable. If the variational order of $\varepsilon$ is nontrivially 2 , we have local 3 rd order Lagrangians arising as $\lambda=h \rho$ from second order $\rho$, or second order Lagrangians if $h \rho$ is $\pi_{3,2^{-}}$ projectable (which, indeed, cannot be reduced to the first order by extracting a variationally trivial Lagrangian). The same order discussion concerns global Lagrangians if $H_{\mathrm{dR}}^{n}(\boldsymbol{Y})=\{0\}$.

Example 3.22. Lagrangians, dynamical forms and Helmholtz-like forms of variational order one:

- Let $n=\operatorname{dim} \boldsymbol{X}=1$. Any $\rho \in \Lambda_{1}^{1} / \Theta_{1}^{1}$ takes the form

$$
\rho=A d t+F_{\sigma} \omega^{\sigma}+C_{\sigma} d \dot{q}^{\sigma} \sim A d t+C_{\sigma} d \dot{q}^{\sigma},
$$

so that

$$
\lambda=h \rho=L d t, \quad \text { where } \quad L=A+C_{\sigma} \ddot{q}^{\sigma} .
$$

We can see that a generic Lagrangian of variational order one in mechanics is of second order, affine in the second derivatives. Then, in particular, reducible second-order Lagrangians (and, of course, first-order Lagrangians) correspond to those classes $[\rho]$ which are generated by $\pi_{1,0^{-}}$ horizontal forms.

If $n=\operatorname{dim} \boldsymbol{X}>1$, we denote $\left.\left.\omega_{i_{1}}=\partial / \partial x^{i_{1}}\right\rfloor \omega_{0}, \omega_{i_{1} i_{2}}=\partial / \partial x^{i_{2}}\right\rfloor \omega_{i_{1}}$, etc.; we have

$$
\rho \sim A \omega_{0}+\sum_{k=1}^{n} C_{\sigma_{1} \ldots \sigma_{k}}^{j_{1} \ldots j_{k}, i_{1} \ldots i_{k}} d y_{j_{1}}^{\sigma_{1}} \wedge \cdots \wedge d y_{j_{k}}^{\sigma_{k}} \wedge \omega_{i_{1} \ldots i_{k}},
$$

where the coefficients $C_{\sigma_{1} \ldots \sigma_{k}}^{j_{1} \ldots j_{k}, i_{1} \ldots i_{k}}$ are totally antisymmetric in the indices $i_{1} \ldots i_{k}$, and totally symmetric in the pairs $\left(\sigma_{1}, j_{1}\right) \ldots\left(\sigma_{k}, j_{k}\right)$. Now, $\lambda=h \rho=L \omega_{0}$, where

$$
L=A+\bar{C}_{\sigma_{1}}^{j_{1}, i_{1}} y_{j_{1} i_{1}}^{\sigma_{1}}+\bar{C}_{\sigma_{1} \sigma_{2}}^{j_{1} j_{2}, i_{1} i_{2}} y_{j_{1} i_{1}}^{\sigma_{1}} y_{j_{2} i_{2}}^{\sigma_{2}}+\cdots+\bar{C}_{\sigma_{1} \ldots \sigma_{n}}^{j_{1} \ldots j_{n}, i_{1} \ldots i_{n}} y_{j_{1} i_{1}}^{\sigma_{1}} \cdots y_{j_{n} i_{n}}^{\sigma_{n}}
$$

\footnotetext{
${ }^{2}$ In the Takens representation $\varepsilon$ is an element of the sheaf of differential forms $\Lambda_{n+1}^{2}$.
} 
(here the $\bar{C}$ 's are just corresponding multipliers of the $C^{\prime}$ 's). Thus, in field theory, a generic Lagrangian of variational order one is of second order, polynomial of degree $n$ in the second derivatives, with coefficients obeying the symmetry-antisymmetry relations described above. In particular, reducible (and first-order) Lagrangians correspond to generating forms $\rho$ that are $\pi_{1,0}$-horizontal. The most famous Lagrangian of this kind is the scalar curvature, giving rise to the Einstein equations.

- Let again $n=\operatorname{dim} \boldsymbol{X}=1$. Any $\rho \in \Lambda_{2}^{1} / \Theta_{2}^{1}$ takes the form

$$
\begin{aligned}
\rho & \sim A_{\sigma}^{1} \omega^{\sigma} \wedge d t+A_{\sigma}^{2} d \omega^{\sigma}+B_{\sigma \nu}^{1} \omega^{\sigma} \wedge d \dot{q}^{\nu}+C_{\sigma \nu} d \dot{q}^{\sigma} \wedge d \dot{q}^{\nu} \\
& \sim \omega^{\sigma} \wedge\left(A_{\sigma} d t+B_{\sigma \nu} d \dot{q}^{\nu}\right)+C_{\sigma \nu} d \dot{q}^{\sigma} \wedge d \dot{q}^{\nu},
\end{aligned}
$$

where $C_{\sigma \nu}=-C_{\nu \sigma}$. In Takens representation we get the dynamical form $\mathcal{I}(\rho)=\varepsilon=E_{\sigma} \omega^{\sigma} \wedge d t$, where

$$
E_{\sigma}=A_{\sigma}+B_{\sigma \nu} \ddot{q}^{\nu}-\frac{d}{d t}\left(2 C_{\sigma \nu} \ddot{q}^{\nu}\right)=A_{\sigma}+\bar{B}_{\sigma \nu} \ddot{q}^{\nu}-2 \frac{\partial C_{\sigma \nu}}{\partial \dot{q}^{\rho}} \ddot{q}^{\nu} \ddot{q}^{\rho}-2 C_{\sigma \nu} \dddot{q}^{\nu} .
$$

Thus a generic dynamical form of variational order one in mechanics is of order three, affine in the third derivatives, and polynomial of degree two in the second derivatives (with a special form of coefficients as above). Second-order dynamical forms of variational order one correspond to generating forms $\rho$ such that $C_{\sigma \nu}=0$, meaning that the corresponding variational class is represented by a $\omega^{\sigma}$-generated form $\rho$ and the differential equations are affine in second derivatives:

$$
\rho \sim A_{\sigma} \omega^{\sigma} \wedge d t+B_{\sigma \nu} \omega^{\sigma} \wedge d \dot{q}^{\nu}, \quad \text { i.e., } \quad E_{\sigma}=A_{\sigma}+B_{\sigma \nu} \ddot{q}^{\nu} .
$$

In particular, if variational $([d \rho]=0$, i.e., $[\rho]=[d \lambda])$, they come from second-order Lagrangians of the form

$$
L=A+C_{\sigma} \ddot{q}^{\sigma}, \quad \frac{\partial C_{\sigma}}{\partial \dot{q}^{\nu}}=\frac{\partial C_{\nu}}{\partial \dot{q}^{\sigma}},
$$

that, indeed, are reducible (equivalent with first-order Lagrangians).

Finally, all first-order dynamical forms are of variational order one and they arise from the class $[\rho]=\left[A_{\sigma} \omega^{\sigma} \wedge d t\right]$.

- Any $\rho \in \Lambda_{3}^{1} / \Theta_{3}^{1}$ takes the form

$$
\begin{aligned}
\rho \sim & A_{\sigma \nu}^{1} \omega^{\sigma} \wedge \omega^{\nu} \wedge d t+A_{\sigma \nu}^{2} \omega^{\sigma} \wedge d \omega^{\nu}+A_{\sigma \nu}^{3} d \dot{q}^{\sigma} \wedge d \omega^{\nu} \\
& +B_{\sigma \nu \rho}^{1} \omega^{\sigma} \wedge \omega^{\nu} \wedge d \dot{q}^{\rho}+B_{\sigma \nu \rho}^{2} \omega^{\sigma} \wedge d \dot{q}^{\nu} \wedge d \dot{q}^{\rho}+C_{\sigma \nu \rho} d \dot{q}^{\sigma} \wedge d \dot{q}^{\nu} \wedge d \dot{q}^{\rho},
\end{aligned}
$$

where $A_{\sigma \nu}^{1}=-A_{\nu \sigma}^{1}, A_{\sigma \nu}^{3}=-A_{\nu \sigma}^{3}, B_{\sigma \nu \rho}^{1}=-B_{\nu \sigma \rho}^{1}, B_{\sigma \nu \rho}^{2}=-B_{\sigma \rho \nu}^{2}$, and the $C$ 's are totally antisymmetric. Then the corresponding Helmholtz-like form becomes $\mathcal{I}(\rho)=\eta=H_{\sigma \nu}^{1} \omega^{\sigma} \wedge \omega^{\nu} \wedge$ $d t+H_{\sigma \nu}^{2} \omega^{\sigma} \wedge \dot{\omega}^{\nu} \wedge d t+H_{\sigma \nu}^{3} \omega^{\sigma} \wedge \ddot{\omega}^{\nu} \wedge d t$, where, as expected, the components have a polynomial structure in second and higher derivatives:

$$
\begin{aligned}
H_{\sigma \nu}^{1} & =A_{\sigma \nu}^{1}+B_{\sigma \nu \rho}^{1} \ddot{q}^{\rho}+\frac{1}{4} \frac{d}{d t}\left(A_{\sigma \nu}^{2}-A_{\nu \sigma}^{2}-2\left(B_{\sigma \nu \rho}^{2}-B_{\nu \sigma \rho}^{2}\right) \ddot{q}^{\rho}\right)-\frac{1}{2} \frac{d^{2}}{d t^{2}}\left(A_{\sigma \nu}^{3}-3 C_{\sigma \nu \rho} \ddot{q}^{\rho}\right) \\
& =\alpha_{\sigma \nu}^{1}+\alpha_{\sigma \nu \rho}^{2} \ddot{q}^{\rho}+\alpha_{\sigma \nu \rho \lambda}^{3} \ddot{q}^{\rho} \ddot{q}^{\lambda}+\alpha_{\sigma \nu \rho \lambda \kappa}^{4} \ddot{q}^{\rho} \ddot{q}^{\lambda} \ddot{q}^{\kappa}+\alpha_{\sigma \nu \rho}^{5} \dddot{q}^{\rho}+\alpha_{\sigma \nu \rho \lambda}^{6} \ddot{q}^{\rho} \ddot{q}^{\lambda}+\frac{3}{2} C_{\sigma \nu \rho} q_{4}^{\rho}, \\
H_{\sigma \nu}^{2} & =-\frac{1}{2}\left(A_{\sigma \nu}^{2}+A_{\nu \sigma}^{2}\right)+\left(B_{\sigma \nu \rho}^{2}+B_{\nu \sigma \rho}^{2}\right) \ddot{q}^{\rho}, \quad H_{\sigma \nu}^{3}=A_{\sigma \nu}^{3}-3 C_{\sigma \nu \rho} \ddot{q}^{\rho} .
\end{aligned}
$$

The requirement that the class $[\rho]$ is $\omega^{\sigma}$-generated gives $A_{\sigma \nu}^{3}=0$ and $C_{\sigma \nu \rho}=0$, and simplifies the Helmholtz-like forms to $H_{\sigma \nu}^{3}=0$, and

$$
H_{\sigma \nu}^{1}=\alpha_{\sigma \nu}^{1}+\alpha_{\sigma \nu \rho}^{2} \ddot{q}^{\rho}+\alpha_{\sigma \nu \rho \lambda}^{3} \ddot{q}^{\rho} \ddot{q}^{\lambda}+\alpha_{\sigma \nu \rho}^{5} \dddot{q}^{\rho} .
$$


If, moreover, $\eta$ is a Helmholtz form (i.e., $[d \rho]=0$ ), related with a $\omega^{\sigma}$-generated class then it corresponds to a second-order dynamical form $\varepsilon=E_{\sigma} \omega^{\sigma} \wedge d t$, where $E_{\sigma}=A_{\sigma}+B_{\sigma \nu} \ddot{q}^{\nu}$, and $B_{\sigma \nu}=B_{\nu \sigma}$.

The above discussion illustrates that the range of applications of the variational sequence is wider than that of corresponding infinite order constructions or even of some other known finite order constructions. Remarkably, it gives solution of the

Inverse order problem (order reduction problem): If a differential $q$-form $\eta$ of order $s$ is (locally/globally) trivial, or (locally/globally) variational (its image under the corresponding morphism $E_{q}$ vanishes) what is the 'minimal order' of the (local/global) preimage $(q-1)$-form $\mu$ such that $\eta=E_{q-1}(\mu)$ ?

Note that this question is answered by the variational sequence, but is not answered, e.g., by the variational bicomplex, or even by Takens representation(!), since $\eta$ is an element of a sheaf of differential forms of order $s$ which contains forms of different variational orders.

Example 3.23. Consider a dynamical form $\varepsilon$ (nontrivially) of order three over a fibred manifold with $n=\operatorname{dim} \boldsymbol{X}=1$. $\varepsilon$ belongs to $\Lambda_{2}^{3}$ and represents a system of 3rd order ordinary differential equations. Takens sequence gives us that if locally variational, $\varepsilon$ comes from a local Lagrangian of order 3. However, from the variational sequence we can get information about the variational order of $\varepsilon$, and hence a finer result about the order of the local Lagrangians. Namely, the following cases may arise:

- The variational order of $\varepsilon$ is (nontrivially) three. This means that in the variational sequence $\varepsilon$ is represented by a class $[\rho] \in \Lambda_{2}^{3} / \Theta_{2}^{3}$. If $\varepsilon$ is locally variational, any minimal order Lagrangian is of order three (not further reducible).

- The variational order of $\varepsilon$ is two. This means that in the variational sequence $\varepsilon$ is represented by a class $[\rho] \in \Lambda_{2}^{2} / \Theta_{2}^{2}$. If locally variational, it has a (local) second-order Lagrangian.

- The variational order of $\varepsilon$ is one. This means that in the variational sequence $\varepsilon$ is represented by a class $[\rho] \in \Lambda_{2}^{1} / \Theta_{2}^{1}$. As follows from the previous example, if $\varepsilon$ is locally variational then the Tonti Lagrangian (which is of order three) is locally reducible to a second-order Lagrangian affine in the second derivatives.

\section{Lie derivative in the variational sequence}

One of the most important features of the geometric formulation of the calculus of variations in jet bundles is the fact that variations can be described by Lie derivatives of differential forms with respect to prolongations of projectable vector fields. A famous example is the first variation formula which takes the integral form [46] (cf. [32])

$$
\left.\left.\int_{\Omega} J^{r} \gamma^{*} L_{J^{r} \Xi} \lambda=\int_{\Omega} J^{2 r-1} \gamma^{*}\left(J^{2 r-1} \Xi\right\rfloor d \rho\right)+\int_{\Omega} J^{2 r-1} \gamma^{*} d\left(J^{2 r-1} \Xi\right\rfloor \rho\right),
$$

where $\rho$ is a Lepage equivalent of $\lambda, \Xi$ is a projectable vector field on $\boldsymbol{Y}$ ("variation vector field"), and $\Omega \subset \boldsymbol{X}$ is a compact connected $n$-dimensional submanifold with boundary. It is, however, even more interesting (and fundamental) that with use of the differential calculus in jet bundles, this formula can be (as mentioned in Section 3.3), invariantly expressed in the "infinitesimal form" as a formula for differential forms on the manifold $J^{2 r} \boldsymbol{Y}$ [46]

$$
\left.\left.L_{J^{r} \Xi} \lambda=h\left(J^{2 r-1} \Xi\right\rfloor d \rho\right)+h d\left(J^{2 r-1} \Xi\right\rfloor \rho\right),
$$


and (with the account of $\lambda=h \rho$ ) it yields a remarkable property of the Lie derivative in the calculus of variations:

$$
L_{J^{2 r} \Xi} h \rho=h L_{J^{2 r-1} \Xi \rho .}
$$

Moreover, as discovered in [62], the Lie derivative commutes with the Euler-Lagrange operator, as the Lie derivative of the Euler-Lagrange form of $\lambda$ is the Euler-Lagrange form of the transformed Lagrangian:

$$
L_{J^{2 r} \Xi} E_{\lambda}=E_{L_{J^{r} \Xi} \lambda}
$$

Such properties of the Lie derivative provide an elegant intrinsic formulation (and an easy proof) of Noether theorem, Noether-Bessel-Hagen theorem [5, 77], conservation laws, and relationship between symmetries of a Lagrangian and its Euler-Lagrange form (for any order and any number of independent variables) $[48,55]$.

Having in mind the variational sequence and its representations, there arises a question about generalization of these properties to variational forms of any degree.

First we recall results obtained in $[56,57,58]$. Let $\mathcal{C}_{r}$ denote the contact ideal of order $r$.

Definition 4.1. A vector field $Z$ on $J^{r} \boldsymbol{Y}$ is called a contact symmetry if it is a symmetry of the contact ideal $\mathcal{C}_{r}$ (meaning that for every contact form $\omega$ the Lie derivative $L_{Z} \omega$ is a contact form.

Contact symmetries were characterized in [56]. It is worth notice that for any projectable vector field $\Xi$ on $\boldsymbol{Y}$ the $r$-jet prolongation $Z=J^{r} \Xi$ is a contact symmetry, and conversely, if a contact symmetry $Z$ on $J^{r} Y$ is projectable onto $\boldsymbol{X}$ then $Z=J^{r} \Xi$ for some $\Xi$ on $\boldsymbol{Y}$.

Let $k \geq 0$. As an immediate consequence of the definition it follows that the Lie derivative of a $k$-contact form by a contact symmetry is an at least $k$-contact form. This means, however, that the Lie derivatives by contact symmetries preserve the sheaves $\Theta_{q}^{r}$.

Proposition 4.2. Let $Z$ be a contact symmetry defined on $J^{r} \boldsymbol{Y}$. Then for all $q \geq 1, L_{Z} \Theta_{q}^{r} \subset \Theta_{q}^{r}$.

Equivalently we can claim that for any two forms $\rho_{1}, \rho_{2}$ belonging to the same class $[\rho]$ in the variational sequence, the Lie derivatives $L_{Z} \rho_{1}, L_{Z} \rho_{2}$ by any contact symmetry $Z$ are also equivalent. Thus, following [57] we can define the Lie derivative $\mathcal{L}_{Z}$ of a class

$$
\mathcal{L}_{Z}[\rho]=\left[L_{Z} \rho\right] .
$$

Now one can easily prove that the property (4.3) extends for any contact symmetry, and through the whole variational sequence, for any morphism $\mathcal{E}_{k}: \Lambda_{k}^{r} / \Theta_{k}^{r} \rightarrow \Lambda_{k+1}^{r} / \Theta_{k+1}^{r}$ [57] (see also [58]):

Theorem 4.3. Let a vector field $Z$ on $J^{r} \boldsymbol{Y}$ be a contact symmetry. Then the Lie derivative $L_{Z}$ commutes with all morphisms in the variational sequence. More explicitly, for all $k \geq 1$

$$
\left.\mathcal{L}_{Z} \mathcal{E}_{k}[\rho]=\mathcal{E}_{k}\left(\mathcal{L}_{Z}[\rho]\right)=\mathcal{E}_{k}[Z\rfloor d \rho\right] .
$$

Proof. Since the Lie derivative commutes with the exterior derivative, we have for any $k$-form $\rho$ on $J^{r} \boldsymbol{Y}$

$$
\left.\mathcal{L}_{Z}[d \rho]=\left[L_{Z} d \rho\right]=\left[d L_{Z} \rho\right]=[d Z\rfloor d \rho\right]
$$

Writing this formula in terms of the morphism $\mathcal{E}_{k}$ we get (4.5). 
From now on, we shall restrict to $\pi_{s}$-projectable contact symmetries (recall that then we have $Z=J^{s} \Xi$ for a projectable vector field $\Xi$ on $\boldsymbol{Y}$ ). From the definition it is clear that the Lie derivative then preserves even individual contact components. Namely, if $Z$ is a projectable contact symmetry on $J^{r+1} \boldsymbol{Y}$ then $Z=J^{r+1} \Xi$ and

$$
L_{J^{r+1} \Xi} p_{k} \rho=p_{k} L_{J^{r} \Xi \rho, \quad k \geq 0,}
$$

generalizing (4.2) to variational forms of any degree. Note that we may write

$$
L_{J^{r+1} \Xi} \pi_{r+1, r}^{*} \rho=L_{J^{r+1} \Xi}\left(\sum_{k=0}^{q} p_{k} \rho\right)=\sum_{k=0}^{q} p_{k}\left(L_{\left.J^{r} \Xi \rho\right) .}\right.
$$

Let us turn back to formula (4.4). It means that the Lie derivative of classes of forms, i.e., variational Lie derivative, can be correctly defined as the equivalence class of the standard Lie derivative of forms, and thus represented by forms. Such a point of view opens a possibility to express the infinitesimal first variation formula (4.1) in terms of the variational morphisms, and, in this way, to obtain a generalization of the first variation formula for any degree of forms (with higher degree analogs of Noether theorems, indeed).

Some results in this direction have been already achieved, namely explicit formulae for the quotient Lie derivative operators were provided, as well as corresponding versions of Noether theorems interpreted in terms of conserved currents for Lagrangians and Euler-Lagrange morphisms. However, only classes of forms up to degree $n+2$, were considered (the latter assumed to be exact) [26]. The representation made use of intrinsic decomposition formulae for vertical morphisms due to Kolář [42], which express geometrically the integration by part procedure, however, in a different way compared to that based on the splitting of the Cartan form [32, 46]. The decomposition formulae introduce local objects such as momenta which could be globalized by means of connections, and besides the usual momentum associated with a Lagrangian, a 'generalized' momentum is associated with an Euler-Lagrange type morphism. Its interpretation in the calculus of variations has not yet been exhaustively exploited; as conjectured in [80], generalized momenta could play a rôle within the multisymplectic framework for field theories.

In the sequel of this section we propose an approach to the problem of representation of the Lie derivative of classes of forms which encompasses previous results and generalizes to any degree of forms. Actually, representation of (non exact) classes of forms of any degree appears relevant, especially in degree $n+3$, for the study of nonvariational differential equations arising from Helmholtz-like forms (related with the problem of the existence of closed $n+2$ forms) [69, 75]. We shall exploit the relation between the interior Euler operator and the Cartan formula for the Lie derivative of differential forms. The representation of the variational Lie derivative will provide, in a quite simple and immediate way, 'any degree' generalizations of (4.1), together with the generalized Noether theorems, as 'quotient Cartan formulae'.

These results, which have an intrinsic importance from a theoretical point of view, as shown in Section 5, have also various concrete applications; besides the ones presented here, it is worth mentioning that applications of iterated variational Lie derivatives in variational problems on gauge-natural bundles produced physically relevant results concerning existence and globality of conserved quantities and on existence of Higgs fields on spinor bundles (see, e.g., [23, 24, 81, $82,83,86])$.

Let $\Xi$ be a projectable vector field on $\boldsymbol{Y}$. We define the interior product of a prolongation of $\Xi$ with the equivalence class of $\rho$ by the formula

$$
\left.\left.J^{r} \Xi\right\rfloor[\rho]=\left[J^{s} \Xi\right\rfloor R_{q}([\rho])\right]
$$

(the equivalence class of the interior product of the vector field with the representation of the equivalence class of $\rho$ ). This definition is well posed since the representation $R_{q}$ is unique. 
Given a $q$-form $\rho$ defined (locally) on $J^{r} \boldsymbol{Y}$, we have a commutative diagram defining an operator $\hat{R}_{q}$

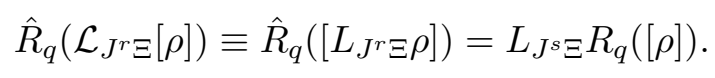

This operator is uniquely defined and is equal, respectively, to the following expressions:

$$
\begin{array}{lll}
L_{J^{s} \Xi} h \rho, & 0 \leq q \leq n, & s=r+1, \\
L_{J^{s} \Xi} \mathcal{I}(\rho), & n+1 \leq q \leq P, & s=2 r+1, \\
L_{J^{s} \Xi \rho,} & q>P+1, & s=r,
\end{array}
$$

where $P$ denotes the corank of the Cartan distribution on $J^{r} \boldsymbol{Y}$.

This definition enables us to deal with ordinary Lie derivatives of forms on $\Lambda_{q}^{s}$, thus we can apply the standard Cartan formula.

We recall a result in [43, Theorem III.11], see also [45].

Lemma 4.4. Let $\Xi$ be a $\pi$-vertical vector field on $\boldsymbol{Y}$ and $\rho$ a differential $q$-form on $J^{r} \boldsymbol{Y}$. Then the following holds true for $i=1, \ldots, q$

$$
\left.\left.J^{r+2} \Xi\right\rfloor p_{i} d p_{i} \rho=-p_{i-1} d\left(J^{r+1} \Xi\right\rfloor p_{i} \rho\right),
$$

and

$$
\left.\left.L_{J^{r+2} \Xi}\left(\pi_{r+2, r+1}\right)^{*} p_{i} \rho=J^{r+2} \Xi\right\rfloor p_{i+1} d p_{i} \rho+p_{i} d\left(J^{r+1} \Xi\right\rfloor p_{i} \rho\right) .
$$

As above, we denote $\omega_{0}=d x^{1} \wedge d x^{2} \wedge \cdots \wedge d x^{n}$. Next, let us denote

$$
\left.\left.\omega_{i}=\frac{\partial}{\partial x^{i}}\right\rfloor \omega_{0}, \quad \omega_{i j}=\frac{\partial}{\partial x^{j}}\right\rfloor \omega_{i} .
$$

It is well known that in order to obtain a representation of classes of degree $n+1$ in the variational sequence by dynamical forms the following integration formula is used [49]

$$
\omega_{J i}^{\alpha} \wedge \omega_{0}=-d \omega_{J}^{\alpha} \wedge \omega_{i},
$$

and the corresponding representation is obtained by taking the $p_{1}$ component obtained by iterated integrations by parts. In order to integrate by parts $(p+k)$-forms with $p<n$, we need to generalize as

$$
\gamma_{\alpha}^{J[i j]} \omega_{J[i}^{\alpha} \wedge \omega_{j]}=-\gamma_{\alpha}^{J[i j]} d \omega_{J}^{\alpha} \wedge \omega_{i j}
$$

This enables us to generalize results given in [45].

Lemma 4.5. Let $\rho \in \Lambda_{p+k}^{r}, 1 \leq p \leq n$. Let $p_{k} \rho=\sum_{|J|=0}^{r} \omega_{J}^{\alpha} \wedge \eta_{\alpha}^{J}$, with $\eta_{\alpha}^{J}(k-1)$-contact $(p+k-1)$-forms. Then we have the decomposition

$$
p_{k} \rho=\mathfrak{I}(\rho)+p_{k} d p_{k} \mathfrak{R}(\rho),
$$

where $\mathfrak{R}(\rho)$ is a local $k$-contact $(p+k-1)$-form such that

$$
\Im(\rho)+p_{k} d p_{k} \mathfrak{R}(\rho)=\omega^{\alpha} \wedge \sum_{|J|=0}^{r}(-1)^{|J|} d_{J} \eta_{\alpha}^{J}+\sum_{|I|=1}^{r} d_{I}\left(\omega^{\alpha} \wedge \zeta_{\alpha}^{I}\right),
$$

with

$$
\zeta_{\alpha}^{I}=\sum_{|J|=0}^{r-|I|}(-1)^{J}\left(\begin{array}{c}
|I|+|J| \\
|J|
\end{array}\right) d_{J} \eta_{\alpha}^{J I}
$$


Notice that $d_{J} \eta_{\alpha}^{J}$ are also $(k-1)$-contact $p$-horizontal (i.e., containing the wedge of $p$ of the forms $\left.d x^{i}\right)(p+k-1)$-forms. It is therefore well defined a local splitting of $p_{k} \rho, \rho \in \Lambda_{p+k}^{r}$, $1 \leq p \leq n$ and $k>1$.

Of course, $\mathfrak{I}=\mathcal{I}$ and $\mathfrak{R}=\mathcal{R}$ in the case $p=n$.

Example 4.6. Let $p=n-1$. We can write $\omega^{\alpha} \wedge \zeta_{\alpha}^{I}=\chi^{I l} \wedge \omega_{l}$, where $\chi^{I l}$ are some local $k$-contact $k$-forms on $J^{2 r} \boldsymbol{Y}$. Therefore

$$
\begin{aligned}
& \sum_{|I|=1}^{r} d_{I}\left(\omega^{\alpha} \wedge \zeta_{\alpha}^{I}\right)=\sum_{|I|=1}^{r} d_{I} \chi^{I l} \wedge \omega_{l}=d_{i} \sum_{|I|=0}^{r-1} d_{I} \chi^{I l i} \wedge \omega_{l}=d_{i} \sum_{|I|=0}^{r-1} d_{I} \chi^{I l i} \wedge d x^{j} \wedge \omega_{l j} \\
& =(-1)^{(n-1)+k-1} d_{i}\left[\sum_{|I|=0}^{r-1}(-1)^{k} d_{I} \chi^{I[l j]} \wedge \omega_{l j}\right] \wedge d x^{i}=d_{H}\left[\sum_{|I|=0}^{r-1}(-1)^{k} d_{I} \chi^{I[l j]} \wedge \omega_{l j}\right] .
\end{aligned}
$$

We denote by $\mathfrak{R}(\rho)=\sum_{|I|=0}^{r-1}(-1)^{k} d_{I} \chi^{I[l j]} \wedge \omega_{l j}$ so that

$$
\sum_{|I|=1}^{r} d_{I}\left(\omega^{\alpha} \wedge \zeta_{\alpha}^{I}\right)=p_{k} d p_{k} \mathfrak{R}(\rho) .
$$

In the following we shall use such a splitting in order to split the vertical differential of a $p$-density and define a corresponding 'momentum form'.

Theorem 4.7. Let $0 \leq q \leq n, \Xi$ a $\pi$-projectable vector field on $\boldsymbol{Y}$ and $\rho$ a differential $q$-form on $J^{r} \boldsymbol{Y}$. We have

1. For $0 \leq q \leq n-1$

$$
\begin{aligned}
\hat{R}_{q}\left(\mathcal{L}_{J^{r} \Xi}[\rho]\right) & \equiv L_{J^{r+1} \Xi} h \rho \\
& \left.\left.\left.\left.=J^{r+2} \Xi_{H}\right\rfloor d_{H} h \rho+J^{r+2} \Xi_{V}\right\rfloor \Im(d \rho)+d_{H}\left(J^{r+1} \Xi_{V}\right\rfloor \tilde{p}_{d_{V} h \rho}+J^{r+1} \Xi_{H}\right\rfloor h \rho\right),
\end{aligned}
$$

where a 'generalized momentum' is defined by

$$
\tilde{p}_{d_{V} h \rho}=-p_{1} \mathfrak{R}(d \rho) .
$$

2. For $q=n$

$$
\left.\left.\left.\hat{R}_{n}\left(\mathcal{L}_{J^{r} \Xi}[\rho]\right) \equiv L_{J^{r+1} \Xi} h \rho=J^{r+2} \Xi_{V}\right\rfloor E_{n}(h \rho)+d_{H}\left(J^{r+1} \Xi_{V}\right\rfloor p_{d_{V} h \rho}+J^{r+1} \Xi_{H}\right\rfloor h \rho\right),
$$

where

$$
p_{d_{V} h \rho}=-p_{1} \mathcal{R}(d \rho) .
$$

Notice, that the latter formula, written as

$$
\left.L_{J^{r+1} \Xi} \lambda=J^{r+2} \Xi_{V}\right\rfloor E_{\lambda}+d_{H} \phi_{\lambda, \Xi}
$$

where

$$
\left.\left.\phi_{\lambda, \Xi}=J^{r+1} \Xi_{V}\right\rfloor p_{d_{V} h \rho}+J^{r+1} \Xi_{H}\right\rfloor h \rho,
$$

is obviously the infinitesimal first variation formula (4.1). We observe that $p_{d_{V} h \rho}$ are momentum forms, and $\phi_{\lambda, \Xi}$ is the Noether current related with $\Xi$ and $\lambda$. 
Proof. For all cases by the Cartan formula

$$
\left.\left.L_{J^{r+1} \Xi} h \rho=J^{r+1} \Xi\right\rfloor d h \rho+d\left(J^{r+1} \Xi\right\rfloor h \rho\right)
$$

we have

$$
\begin{aligned}
L_{J^{r+1}} \Xi \rho \rho & \left.\left.=\left(J^{r+2} \Xi_{H}+J^{r+2} \Xi_{V}\right)\right\rfloor\left(d_{V} h \rho+d_{H} h \rho\right)+\left(d_{V}+d_{H}\right)\left(\left(J^{r+1} \Xi_{H}+J^{r+1} \Xi_{V}\right)\right\rfloor h \rho\right) \\
& \left.\left.\left.=d_{H}\left(J^{r+1} \Xi_{H}\right\rfloor h \rho\right)+J^{r+2} \Xi_{H}\right\rfloor d_{H} h \rho+J^{r+2} \Xi_{V}\right\rfloor d_{V} h \rho .
\end{aligned}
$$

This expression can be further characterized in more detail.

Let $0 \leq q \leq n-1$. Since $d_{V} h \rho=p_{1} d \rho=\mathfrak{I}(d \rho)+p_{1} d p_{1} \mathfrak{R}(d \rho)$, and since by Lemma 4.4,

$$
\left.\left.\left.J^{r+2} \Xi_{V}\right\rfloor p_{1} d p_{1} \mathfrak{R}(d \rho)=-p_{0} d\left(J^{r+1} \Xi_{V}\right\rfloor p_{1} \mathfrak{R}(d \rho)\right)=-d_{H}\left(J^{r+1} \Xi_{V}\right\rfloor p_{1} \mathfrak{R}(d \rho)\right),
$$

we can define a momentum form associated with a density of degree $<n$ by (4.7) and obtain

$$
\begin{aligned}
L_{J^{r+1}} h \rho & \left.\left.\left.=d_{H}\left(J^{r+1} \Xi_{H}\right\rfloor h \rho\right)+J^{r+2} \Xi_{H}\right\rfloor d_{H} h \rho+J^{r+2} \Xi_{V}\right\rfloor\left(\mathfrak{I}(d \rho)+p_{1} d p_{1} \mathfrak{R}(d \rho)\right) \\
& \left.\left.\left.\left.=J^{r+2} \Xi_{V}\right\rfloor \mathfrak{I}(d \rho)+J^{r+2} \Xi_{H}\right\rfloor d_{H} h \rho+d_{H}\left(J^{r+1} \Xi_{V}\right\rfloor \tilde{p}_{d_{V} h \rho}+J^{r+1} \Xi_{H}\right\rfloor h \rho\right) .
\end{aligned}
$$

Let $q=n$. Then $d_{H} h \rho=0$, hence $\left.J^{r+2} \Xi_{H}\right\rfloor d_{H} h \rho=0$. Next, again since $d_{V} h \rho=p_{1} d \rho=$ $\mathcal{I}(d \rho)+p_{1} d p_{1} \mathcal{R}(d \rho)$ and since by Lemma $\left.\left.4.4, J^{r+2} \Xi_{V}\right\rfloor p_{1} d p_{1} \mathcal{R}(d \rho)=-d_{H}\left(J^{r+1} \Xi_{V}\right\rfloor p_{1} \mathcal{R}(d \rho)\right)$, we define a local momentum form by (4.8), and obtain

$$
\begin{aligned}
L_{J^{r+1} \Xi} h \rho & \left.\left.=J^{r+2} \Xi_{V}\right\rfloor\left(\mathcal{I}(d \rho)-d_{H}\left(J^{r+1} \Xi_{V}\right\rfloor p_{1} \mathcal{R}(d \rho)\right)+d_{H}\left(J^{r+1} \Xi_{H}\right\rfloor h \rho\right) \\
& \left.\left.\left.=J^{r+2} \Xi_{V}\right\rfloor \mathcal{I}(d \rho)+d_{H}\left(J^{r+1} \Xi_{V}\right\rfloor p_{d_{V}} h \rho+J^{r+1} \Xi_{H}\right\rfloor h \rho\right) \\
& \left.=J^{r+2} \Xi_{V}\right\rfloor \mathcal{I}(d \rho)+d_{H} \phi_{\lambda, \Xi} .
\end{aligned}
$$

However, $\phi_{\lambda, \Xi}$ is a horizontal form, and in Takens representation, $\mathcal{I}(d \rho)=E_{n}(h \rho)$ and $d_{H} \phi_{\lambda, \Xi}=$ $h d \phi_{\lambda, \Xi}=E_{n-1}\left(\phi_{\lambda, \Xi}\right)$, proving the theorem.

Theorem 4.8. Let $q=n+k, k \geq 1$. Let $\Xi$ be a $\pi$-vertical vector field on $\boldsymbol{Y}$. We have

$$
\left.\left.\hat{R}_{n+k}\left(\mathcal{L}_{J^{r} \Xi}[\rho]\right)=J^{s+1} \Xi\right\rfloor p_{k+1} d(\mathcal{I}(\rho))+p_{k} d\left(J^{s} \Xi\right\rfloor \mathcal{I}(\rho)\right) .
$$

Proof. For simplicity of notation, in what follows we omit the projections (so we write $p_{k} \rho$ instead of $\pi_{s, r+1}^{*} p_{k} \rho$, etc.).

Since $R_{n+k}([\rho])=\mathcal{I}(\rho)=p_{k} \rho-p_{k} d p_{k} \mathcal{R}(\rho)$, we have

$$
\hat{R}_{n+k}\left(\mathcal{L}_{J^{r} \Xi}[\rho]\right)=L_{J^{s} \Xi} \mathcal{I}(\rho)=L_{J^{s} \Xi p_{k} \rho-L_{J^{s}} \Xi p_{k}} d p_{k} \mathcal{R}(\rho) .
$$

By applying Lemma 4.4 to $d p_{k} \mathcal{R}(\rho)$ we have

$$
\left.\left.L_{J^{s+1} \Xi} p_{k} d p_{k} \mathcal{R}(\rho)=J^{s+1} \Xi\right\rfloor p_{k+1} d p_{k} d p_{k} \mathcal{R}(\rho)+p_{k} d\left(J^{s} \Xi\right\rfloor p_{k} d p_{k} \mathcal{R}(\rho)\right) .
$$

Simple manipulations show that

$$
\left.\left.p_{k} d\left(J^{s} \Xi\right\rfloor p_{k} d p_{k} \mathcal{R}(\rho)\right)=-J^{s+1} \Xi\right\rfloor p_{k+1} d p_{k} d p_{k} \mathcal{R}(\rho),
$$

so that

$$
L_{J^{s+1} \Xi} p_{k} d p_{k} \mathcal{R}(\rho)=0 .
$$

On the other hand

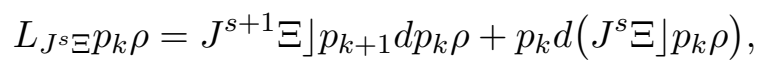


but, since $p_{k} \rho=\mathcal{I}(\rho)+p_{k} d p_{k} \mathcal{R}(\rho)$, by substituting we get

$$
\begin{aligned}
\left.J^{s+1} \Xi\right\rfloor p_{k+1} d p_{k} \rho & \left.=J^{s+1} \Xi\right\rfloor p_{k+1} d\left(\mathcal{I}(\rho)+p_{k} d p_{k} \mathcal{R}(\rho)\right) \\
& \left.\left.=J^{s+1} \Xi\right\rfloor p_{k+1} d(\mathcal{I}(\rho))+J^{s+1} \Xi\right\rfloor p_{k+1} d\left(p_{k} d p_{k} \mathcal{R}(\rho)\right) \\
& \left.\left.=J^{s+1} \Xi\right\rfloor p_{k+1} d(\mathcal{I}(\rho))-p_{k} d\left(J^{s} \Xi\right\rfloor p_{k} d p_{k} \mathcal{R}(\rho)\right) .
\end{aligned}
$$

Again by the same substitution in $\left.p_{k} d\left(J^{s} \Xi\right\rfloor p_{k} \rho\right)$, we get

$$
\left.\left.p_{k} d\left(J^{s} \Xi\right\rfloor \mathcal{I}(\rho)\right)+p_{k} d\left(J^{s} \Xi\right\rfloor p_{k} d p_{k} \mathcal{R}(\rho)\right) .
$$

Summing up we finally obtain

$$
\left.\left.\hat{R}_{n+k}\left(\mathcal{L}_{J^{r} \Xi}[\rho]\right)=L_{J^{s} \Xi} p_{k} \rho=J^{s+1} \Xi\right\rfloor p_{k+1} d(\mathcal{I}(\rho))+p_{k} d\left(J^{s} \Xi\right\rfloor \mathcal{I}(\rho)\right) .
$$

By the above theorem we get a generalization of the famous Noether equation and NoetherBessel-Hagen equation (i.e., equation for symmetries of a Lagrangian and of the Euler-Lagrange form) to a source form of any degree. Indeed, a projectable vector field $\Xi$ on $\boldsymbol{Y}$ is a symmetry of $\mathcal{I}(\rho)$, i.e., $L_{J^{s} \Xi} \mathcal{I}(\rho)=0$ if and only if $L_{J^{s+1} \Xi_{V}} \mathcal{I}(\rho)=0$, that is if and only if

$$
\left.\left.J^{s+1} \Xi_{V}\right\rfloor p_{k+1} d(\mathcal{I}(\rho))+p_{k} d\left(J^{s} \Xi_{V}\right\rfloor \mathcal{I}(\rho)\right)=0 .
$$

Theorems 4.7 and 4.8 are the desired generalization of the infinitesimal first variation formula (4.1) to forms of any degree. The 'boundary term' on the right-hand side in Theorem 4.7 contains 'generalized momenta', and provides a lower-degree analog of Noether theorem in the same way as in the classical case $q=n(k=0)$.

Lemma 4.9. Let $q=n+k, k \geq 1$, and let $\Xi$ be a $\pi$-projectable vector field on $\boldsymbol{Y}$. We have for every class $[\rho]$ of degree $n+k$

$$
\mathcal{L}_{J^{r+1} \Xi_{H}}[\rho]=0 .
$$

Proof. In fact, $\left.J^{s+1} \Xi_{H}\right\rfloor d_{H} \mathcal{I}(\rho)=0$ because $d_{H} \mathcal{I}(\rho)$ is the horizontal differential of an $n$ horizontal form. On the other hand since $d_{V} \mathcal{I}(\rho)=p_{k+1} d(\mathcal{I}(\rho))$ and $p_{k+1} d \circ p_{k+1} d=0$, we have $\left.\left.J^{s+1} \Xi_{H}\right\rfloor d_{V} \mathcal{I}(\rho)=J^{s+1} \Xi_{H}\right\rfloor p_{k+1} d(\mathcal{I}(\rho))($ a $(k+1)$-contact and $(n-1)$-horizontal $(n+k)$-form); therefore $\left.\left.\left.\hat{R}_{n+k}\left(\mathcal{L}_{J^{r+1} \Xi_{H}}[\rho]\right)=J^{s+1} \Xi_{H}\right\rfloor p_{k+1} d(\mathcal{I}(\rho))\right)+p_{k} d\left(J^{s+1} \Xi_{H}\right\rfloor \mathcal{I}(\rho)\right) \in \Theta_{n+k}^{s+1}$.

Since $p_{k+1} d(\mathcal{I}(\rho))=\mathcal{I}(d(\mathcal{I}(\rho)))=R_{q+1}\left(\mathcal{E}_{q}([\rho])\right)$, taking into account Theorem 3.15, we finally obtain a 'quotient' Cartan formula for the variational Lie derivative of $n+k$ classes, with $k \geq 1$.

Theorem 4.10. Let $k \geq 1$. Let $\Xi$ be a $\pi$-projectable vector field on $\boldsymbol{Y}$. For every class $[\rho] \in \Lambda_{n+k}^{r} / \Theta_{n+k}^{r}$ we have (up to pull-backs)

$$
\left.\left.\mathcal{L}_{J^{r} \Xi}[\rho]=J^{r+1} \Xi_{V}\right\rfloor \mathcal{E}_{n+k}([\rho])+\mathcal{E}_{n+k-1}\left(J^{r+1} \Xi_{V}\right\rfloor[\rho]\right) .
$$

Proof. Since $\Xi$ is assumed to be a $\pi$-projectable vector field on $\boldsymbol{Y}$, up to projections,

$$
\hat{R}_{n+k}\left(\mathcal{L}_{J^{r} \Xi}[\rho]\right)=\hat{R}_{n+k}\left(\mathcal{L}_{J^{r+1} \Xi_{V}}[\rho]\right)+\hat{R}_{n+k}\left(\mathcal{L}_{J^{r+1} \Xi_{H}}[\rho]\right)
$$

holds true and the result follows from the above lemma and theorem (the latter applied for the vertical part of $\left.J^{r} \Xi\right)$. 


\section{Some applications}

\subsection{Helmholtz forms in mechanics}

As mentioned above, for $k \geq 2$, a class $[\rho] \in \mathcal{V}_{n+k}^{r}$ in the variational sequence can be represented by different source forms. We shall illustrate this feature on the image $\mathcal{E}_{n+1}([\varepsilon])=[d \varepsilon] \in \mathcal{V}_{n+2}^{2}$, where $\varepsilon$ is a second-order dynamical form, in the case when $n=1$ (mechanics).

Let $n=\operatorname{dim} X=1$; as above, denote local fibred coordinates on $\boldsymbol{Y}$ by $\left(t, q^{\sigma}\right)$ and the associated coordinates on $J^{3} \boldsymbol{Y}$ by $\left(t, q^{\sigma}, \dot{q}^{\sigma}, \ddot{q}^{\sigma}, \dddot{q}^{\sigma}\right)$, and let

$$
\omega^{\sigma}=d q^{\sigma}-\dot{q}^{\sigma} d t, \quad \dot{\omega}^{\sigma}=d \dot{q}^{\sigma}-\ddot{q}^{\sigma} d t, \quad \ddot{\omega}^{\sigma}=d \ddot{q}^{\sigma}-\dddot{q}^{\sigma} d t .
$$

Consider a dynamical form $\varepsilon \in \Lambda_{2}^{2}, \varepsilon=E_{\sigma} \omega^{\sigma} \wedge d t$. Then $[d \varepsilon]$ is represented by the following canonical source form (called canonical Helmholtz form), obtained by a straightforward calculation:

$$
\begin{aligned}
H_{\varepsilon}= & \mathcal{I}(d \varepsilon)=\frac{1}{2}\left(\left(\frac{\partial E_{\sigma}}{\partial q^{\nu}}-\frac{\partial E_{\nu}}{\partial q^{\sigma}}-\frac{1}{2} \frac{d}{d t}\left(\frac{\partial E_{\sigma}}{\partial \dot{q}^{\nu}}-\frac{\partial E_{\nu}}{\partial \dot{q}^{\sigma}}\right)+\frac{1}{2} \frac{d^{2}}{d t^{2}}\left(\frac{\partial E_{\sigma}}{\partial \ddot{q}^{\nu}}-\frac{\partial E_{\nu}}{\partial \ddot{q}^{\sigma}}\right)\right) \omega^{\nu}\right. \\
& \left.+\left(\frac{\partial E_{\sigma}}{\partial \dot{q}^{\nu}}+\frac{\partial E_{\nu}}{\partial \dot{q}^{\sigma}}-2 \frac{d}{d t} \frac{\partial E_{\nu}}{\partial \ddot{q}^{\sigma}}\right) \dot{\omega}^{\nu}+\left(\frac{\partial E_{\sigma}}{\partial \ddot{q}^{\nu}}-\frac{\partial E_{\nu}}{\partial \ddot{q}^{\sigma}}\right) \ddot{\omega}^{\nu}\right) \wedge \omega^{\sigma} \wedge d t .
\end{aligned}
$$

By construction $H_{\varepsilon} \in \Lambda_{3}^{5}$, however, it is projectable to $J^{4} \boldsymbol{Y}$.

From the exactness of the representation sequence we get that the condition $E_{2}(\varepsilon)=H_{\varepsilon}=0$ is necessary and sufficient for existence of a local Lagrangian $\lambda$ such that $\varepsilon=E_{1}(\lambda)=E_{\lambda}$. Expressed in terms of the components of $H_{\varepsilon}$ the condition $H_{\varepsilon}=0$ gives the celebrated Helmholtz conditions of local variationality for dynamical forms, in the context of the variational sequence called also source constraints.

The canonical Helmholtz form is not the unique source form providing Helmholtz conditions. Another distinguished Helmholtz form is [53]

$$
\begin{aligned}
\bar{H}_{\varepsilon}= & \frac{1}{2}\left(\left(\frac{\partial E_{\sigma}}{\partial q^{\nu}}-\frac{\partial E_{\nu}}{\partial q^{\sigma}}-\frac{1}{2} \frac{d}{d t}\left(\frac{\partial E_{\sigma}}{\partial \dot{q}^{\nu}}-\frac{\partial E_{\nu}}{\partial \dot{q}^{\sigma}}\right)\right) \omega^{\nu}\right. \\
& \left.+\left(\frac{\partial E_{\sigma}}{\partial \dot{q}^{\nu}}+\frac{\partial E_{\nu}}{\partial \dot{q}^{\sigma}}-\frac{d}{d t}\left(\frac{\partial E_{\sigma}}{\partial \ddot{q}^{\nu}}+\frac{\partial E_{\nu}}{\partial \ddot{q}^{\sigma}}\right)\right) \dot{\omega}^{\nu}+\left(\frac{\partial E_{\sigma}}{\partial \ddot{q}^{\nu}}-\frac{\partial E_{\nu}}{\partial \ddot{q}^{\sigma}}\right) \ddot{\omega}^{\nu}\right) \wedge \omega^{\sigma} \wedge d t .
\end{aligned}
$$

$\bar{H}_{\varepsilon}$ is of order 3 , and is, indeed, equivalent with $H_{\varepsilon}$. Note that it provides Helmholtz conditions in an equivalent and more simple form. The relationship between the canonical and the "reduced" Helmholtz form is as follows [70]:

$$
\bar{H}_{\varepsilon}=H_{\varepsilon}+p_{2} d \eta
$$

where

$$
\eta=-\frac{1}{4} \frac{d}{d t}\left(\frac{\partial E_{\sigma}}{\partial \ddot{q}^{\nu}}-\frac{\partial E_{\nu}}{\partial \ddot{q}^{\sigma}}\right) \omega^{\nu} \wedge \omega^{\sigma}
$$

It is worth note that in the most frequent case when the components of $\varepsilon$ are affine in the second derivatives with symmetric coefficients, i.e.,

$$
E_{\sigma}=A_{\sigma}+B_{\sigma \nu} \ddot{q}^{\nu}, \quad B=B^{T},
$$

the canonical Helmholtz form $H_{\varepsilon}$ is projectable to $J^{3} \boldsymbol{Y}$ and coincides with the "reduced" form $\bar{H}_{\varepsilon}$. 
Asking about Helmholtz forms of variational order 1, that is, coming from the variational sequence of order 1, we get the following result: The Helmholtz form $H_{\varepsilon}$ has variational order 1 if and only if it is projectable onto $J^{2} \boldsymbol{Y}$, and this is if and only if the following conditions are satisfied:

$$
B=B^{T}, \quad \frac{\partial B_{\sigma \nu}}{\partial \dot{q}^{\rho}}=\frac{\partial B_{\sigma \rho}}{\partial \dot{q}^{\nu}} .
$$

Note that:

- Conditions (5.1) are a part of the Helmholtz conditions, i.e., they are necessary for $\varepsilon$ be locally variational.

- Conditions (5.1) are integrability conditions, guaranteeing existence of a function $L$ such that

$$
B_{\sigma \nu}=-\frac{\partial^{2} L}{\partial \dot{q}^{\sigma} \partial \dot{q}^{\nu}}
$$

(the minus sign is chosen just for convenience). $L$ is not generally a Lagrangian for $\varepsilon$ (indeed, the form $\varepsilon$ need not be variational, $H_{\varepsilon}$ need not be equal to 0 ). However, with help of the Euler-Lagrange form $E_{\lambda}$ of $\lambda=L d t$ we get $\varepsilon=E_{\lambda}-F$, so that in coordinates equations represented by $\varepsilon$ take the form

$$
\frac{\partial L}{\partial y^{\sigma}}-\frac{d}{d t} \frac{\partial L}{\partial \dot{y}^{\sigma}}=F_{\sigma}
$$

We can conclude that the requirement that $H_{\varepsilon}$ has variational order one means that the corresponding equations are so-called Lagrange equations of the second kind.

- It can be easily shown that the above equations are variational, i.e., $H_{\varepsilon}=0$ if and only if the force $F$ has the form of a Lorentz-like force (as, e.g., Lorentz force, Coriolis force, etc.).

\subsection{Inverse problems in the variational sequence}

Perhaps the best known problems which are solved with help of variational sequences and bicomplexes are the local and global inverse problem of the calculus of variations, and the problem of local and global triviality of Lagrangians. Let us show how these problems appear and are solved with help of the variational sequence and the representation sequences.

- Variationally trivial Lagrangians, also called null-Lagrangians are Lagrangians which give rise to identically zero Euler-Lagrange expressions (meaning that the Euler-Lagrange form $E_{\lambda}$ identically vanishes). This problem concerns the classes of degree $n-1, n$ and $n+1$ and the corresponding morphisms $\mathcal{E}_{n-1}: \mathcal{V}_{n-1}^{r} \rightarrow \mathcal{V}_{n}^{r}$, and $\mathcal{E}_{n}: \mathcal{V}_{n}^{r} \rightarrow \mathcal{V}_{n+1}^{r}$ (the Euler-Lagrange mapping).

Consider the variational sequence $0 \rightarrow \mathbb{R}_{\boldsymbol{Y}} \rightarrow \mathcal{V}_{*}^{r}$, and assume that a class $[\rho] \in \mathcal{V}_{n}^{r}$ satisfies $\mathcal{E}_{n}([\rho])=0$. Then by exactness of the variational sequence there exists a class $[\eta] \in \mathcal{V}_{n-1}^{r}$ such that $\mathcal{E}_{n-1}([\eta])=[\rho]=[d \eta]$. The condition $\mathcal{E}_{n}([\rho])=0$ is the local variational triviality condition and determines the structure of Lagrangians whose Euler-Lagrange forms vanish identically: every variationally trivial Lagrangian locally is a closed $n$-form $d \eta$ modulo contact forms, in other words, $\lambda=h d \eta=d_{H} \eta$. In coordinates, if $\eta=f^{i} \omega_{i}+\cdots$ then $L=d_{i} f^{i}$. If, in addition, $H_{\mathrm{dR}}^{n} \boldsymbol{Y}=\{0\}$ then $\eta$ may be chosen globally defined on $J^{r} \boldsymbol{Y}$.

In Takens representation the same result follows by the following arguments: If $[\rho] \in \mathcal{V}_{n}^{r}$ satisfies $\mathcal{E}_{n}([\rho])=0$ then $\lambda=R_{n}([\rho])=h \rho$ satisfies the constraint on local variational triviality $E_{n}(h \rho)=0$, which means that locally $h \rho=R_{n-1}[(d \eta])=h d \eta$. A corresponding local Lagrangian 
is then $\lambda=R_{n}([\eta])=h \eta$. Condition $H_{\mathrm{dR}}^{n} \boldsymbol{Y}=\{0\}$ then guarantees existence of a global $\eta$, hence of a global Lagrangian $h \eta$.

In Lepage representation, the local triviality condition $\mathcal{E}_{n}([\rho])=0$ means that (any) Lepage $n$-form $\rho \in[\rho]$ (Lepage equivalent of $\lambda=h \rho$ ) is closed. Hence locally $\rho=d \eta$, i.e., $\lambda=h d \eta=$ $\left(d_{H} \eta=\right) h d h \eta=d_{i} f^{i} \omega_{0}$, and $\eta$, resp. the relevant part $h \eta$, is constructed from $\rho$ with help of the Poincaré contact homotopy operator as $\eta=\mathcal{A} \rho$, resp. $h \eta=f^{i} \omega_{i}=\mathcal{A} \lambda$.

- By the inverse problem of the calculus of variations, here one understands the question about local and global variationality of a dynamical form $\varepsilon$. This means the problem to determine constraints on variationality (Helmholtz conditions), and for a locally variational form, to construct a local Lagrangian. This problem concerns the classes of degree $n, n+1$ and $n+2$ in the variational sequence, and the corresponding morphisms $\mathcal{E}_{n}: \mathcal{V}_{n}^{r} \rightarrow \mathcal{V}_{n+1}^{r}$ (the Euler-Lagrange morphism) and $\mathcal{E}_{n+1}: \mathcal{V}_{n+1}^{r} \rightarrow \mathcal{V}_{n+2}^{r}$ (the Helmholtz morphism).

Consider the variational sequence $0 \rightarrow \mathbb{R}_{\boldsymbol{Y}} \rightarrow \mathcal{V}_{*}^{r}$, and assume that a class $[\rho] \in \mathcal{V}_{n+1}^{r}$ satisfies $\mathcal{E}_{n+1}([\rho])=0$. Then by exactness of the variational sequence there exists a class $[\eta] \in \mathcal{V}_{n}^{r}$ such that $\mathcal{E}_{n}([\eta])=[\rho]=[d \eta]$. The condition $\mathcal{E}_{n+1}([\rho])=0$ is the local variationality condition and determines the structure of locally variational forms (giving rise to variational equations): Every locally variational form $\varepsilon$ of variational order $r$ is a closed $(n+1)$-form $d \eta$ modulo strongly contact forms and exterior derivatives of contact forms of order $r$. Remarkably, this factorization procedure determines the structure of locally variational forms of the variational order $r$, i.e., produces constraints for a locally variational form of order $s$ to come from a local Lagrangian of order $r$ (order reduction constraints). If, in addition, $H_{\mathrm{dR}}^{n+1} \boldsymbol{Y}=\{0\}$ then $\varepsilon$ is globally variational.

In Takens representation, if $[\rho] \in \mathcal{V}_{n+1}^{r}$ satisfies $\mathcal{E}_{n+1}([\rho])=0$ then the dynamical form $\varepsilon=R_{n+1}([\rho])=\mathcal{I} \rho$ satisfies $E_{n+1}(\varepsilon)=0$ (Helmholtz conditions). Hence we can say:

A dynamical form $\varepsilon$ is locally variational if and only if its Helmholtz form $H_{\varepsilon}=E_{n+1}(\varepsilon)$ vanishes.

Then locally $\varepsilon=R_{n}[(d \eta])=\mathcal{I} d \eta$, providing a Lagrangian for $\varepsilon$.

In Lepage representation, the local variationality condition $\mathcal{E}_{n+1}([\rho])=0$ means that (any) Lepage form $\rho \in[\rho]$ (Lepage equivalent of $\varepsilon=\mathcal{I} \rho=p_{1} d \rho$ ) is closed. Hence locally $\rho=d \eta$, i.e., $\varepsilon=p_{1} \rho=p_{1} d \eta$, and $\eta$, respectively, a Lagrangian $\lambda$ for $\varepsilon$ is constructed from $\rho$ with help of the Poincaré contact homotopy operator as $\eta=A \rho$, respectively, $\lambda=\mathcal{A} \varepsilon$ (Tonti Lagrangian).

In $[69,75]$ the same questions concerning $(n+2)$-forms have been studied for the case of mechanics ( $\operatorname{dim} \boldsymbol{X}=1)$. Among others, explicit formulae for constraints on local 'variational triviality' of Helmholtz-like forms, $E_{n+2}(\rho)=0$, generalizing Helmholtz conditions to forms of degree $n+2$, as well as Tonti-like formulas for the corresponding (nonvariational) dynamical forms, have been found.

In the same way, the inverse problems, the equivalence problems, and the corresponding order reduction problems in the local and global form extend to each column of the variational sequence, and are solved by the exactness of the sequence. A practical question, however, is to find explicit formulae for source constraints, i.e., constraints on a source form (of any degree) to be variationally trivial (i.e., to belong to the kernel of the corresponding morphism in the variational sequence). The problem in its full generality is solved easily with help of the Lepage representation of the variational sequence, where it is transformed to the Poincaré lemma:

Theorem 5.1 (general inverse variational problem). Let $\varepsilon$ be a source form of degree $n+k$, $k \geq 1$. The following conditions are equivalent:

(1) $\varepsilon$ is locally variationally trivial, i.e., belongs to Ker $E_{n+k}$,

(2) $\varepsilon$ has a closed Lepage equivalent,

(3) every Lepage equivalent $\alpha$ of $\varepsilon$ satisfies $p_{k+1} d \alpha=0$. 
If $\varepsilon$ is locally variationally trivial then a corresponding local primitive source $(n+k-1)$-form $\eta$ (satisfying $E_{n+k-1}(\eta)=\varepsilon$ ) is $\eta=\mathcal{A} \varepsilon$.

If $H_{\mathrm{dR}}^{n+k} \boldsymbol{Y}=\{0\}$ then $\varepsilon$ is globally variationally trivial, with a global primitive of order $\leq 2 r+1$, where $r$ is the variational order of $\varepsilon$.

Proof. Let $\varepsilon \in \operatorname{Ker} E_{n+k}$. This means that $\varepsilon=\mathcal{I}(\rho)$ where $[\rho] \in \operatorname{Ker} \mathcal{E}_{n+k}$. Hence $[\rho]=[d \nu]=$ $\mathcal{E}_{n+k-1}([\nu])$, and we have $\left\{\operatorname{Lep}_{n+k}(\varepsilon)\right\}=\tilde{R}_{n+k}(\rho)=\tilde{R}_{n+k}(d \nu)=\left\{\operatorname{Lep}_{n+k}\left(R_{n+k}(d \nu)\right)\right\}=\{0\}$, proving (2).

Next, assume $d\left\{\operatorname{Lep}_{n+k}(\varepsilon)\right\}=\{0\}$. This means that every Lepage equivalent $\alpha$ of $\varepsilon$ satisfies $d \alpha=d \mu$ where $\mu$ is at least $(k+2)$-contact. Hence $p_{k+1} d \alpha=0$, as desired.

Finally, assume (3). Since $\alpha=\theta+d \nu+\mu$ where $\mu$ is at least $(k+2)$-contact, we can see that $p_{k+1} d \theta=0$. Hence $\{\alpha\}=\tilde{R}([\rho])$ where $\{d \alpha\}=\tilde{R}([d \rho])=\{0\}$. This means that $[d \rho]=[0]$, i.e., $[\rho] \in \operatorname{Ker} \mathcal{E}_{n+k}$, and $\varepsilon=\mathcal{I}(\rho) \in \operatorname{Ker} E_{n+k}$.

The remaining assertions follow immediately from the properties of $\mathcal{A}, \mathcal{I}$, and the variational sequence.

Condition (3) in Theorem 5.1 can be also expressed by means of the Cartan $(n+k)$-form $\theta_{\varepsilon}$ of the source $(n+k)$-form $\varepsilon$ as

$$
p_{k+1} d \theta_{\varepsilon}=0 .
$$

It represents necessary and sufficient source constraints (constraints on a source form to be locally variationally trivial) for source forms of any degree $q>n$, and of any order. For $q=n+1$ this is exactly an intrinsic form of the celebrated Helmholtz conditions mentioned above.

\subsection{Bosonic string}

A bosonic string is described as a minimal immersion of a 2-dimensional surface in a 4-dimensional pseudo-Riemannian manifold $(M, g)$. Hence the 'motion equation' is the minimal surface equation arising from the area functional (known as Nambu-Goto action [35]).

The corresponding fibred manifold is $\pi: \boldsymbol{Y} \rightarrow \boldsymbol{X}$ where $\boldsymbol{X}=\mathbb{R}^{2}$ and $\boldsymbol{Y}=\mathbb{R}^{2} \times M$, so that $\operatorname{dim} \boldsymbol{Y}=6$. Base coordinates $\tau^{0}$ and $\tau^{1}$ represent the time evolution parameter and the position on the string, respectively, fibred coordinates $\left(x^{\mu}\right), \mu=0,1,2,3$, are local coordinates on $M$. We denote by $\left(\tau^{i}, x^{\mu}, x_{i}^{\mu}\right)$, where $i=0,1$ and $\mu=0,1,2,3$, associated local coordinates on $J^{1} \boldsymbol{Y}$, and put $\omega^{\mu}=d x^{\mu}-x_{i}^{\mu} d \tau^{i}$. If $\iota: \mathbb{R}^{2} \rightarrow \Sigma \subset M$ is an immersion of $\mathbb{R}^{2}$ to $M$, and $g=g_{\mu \nu} d x^{\mu} \otimes d x^{\nu}$ is a (symmetric regular pseudo-Riemannian) metric on $M$, we have the induced metric on $\Sigma$

$$
\iota^{*} g \equiv h_{i j} d \tau^{i} \otimes d \tau^{j}=\left(g_{\mu \nu} \circ \iota\right) \frac{\partial x^{\mu}}{\partial \tau^{i}} \frac{\partial x^{\nu}}{\partial \tau^{j}} d \tau^{i} \otimes d \tau^{j}
$$

and the area element

$$
d \Sigma=\sqrt{-\operatorname{det}\left(h_{i j}\right)} d \tau^{0} \wedge d \tau^{1}=\sqrt{-\operatorname{det}\left(\left(g_{\mu \nu} \circ \iota\right) \frac{\partial x^{\mu}}{\partial \tau^{i}} \frac{\partial x^{\nu}}{\partial \tau^{j}}\right)} d \tau^{0} \wedge d \tau^{1},
$$

hence the Nambu-Goto action reads (in units with light speed $c=1$ )

$$
S=-T \int_{\Sigma} d \Sigma
$$

where $T$ is the (constant) string tension. In the fibred setting, we write the action as $S=$ $\int_{\Omega} J^{1} \gamma^{*} \lambda$, where $\Omega$ is a domain in $\mathbb{R}^{2}, \gamma=\left(\operatorname{id}_{\mathbb{R}^{2}}, \iota\right)$ is a local section of $\pi$, and the Lagrangian (now considered as a horizontal form on $J^{1} \boldsymbol{Y}$ ) is

$$
\lambda=-T \sqrt{-\operatorname{det}\left(g_{\mu \nu} x_{i}^{\mu} x_{j}^{\nu}\right)} d \tau^{0} \wedge d \tau^{1}
$$


(note that the action is reparametrization invariant). Let us denote

$$
L\left(\tau^{i}, x^{\mu}, x_{i}^{\mu}\right)=-T \sqrt{-D}=-T \sqrt{-\operatorname{det}\left(g_{\mu \nu} x_{i}^{\mu} x_{j}^{\nu}\right)}=-T \sqrt{\left(g_{\alpha \beta} g_{\mu \nu}-g_{\alpha \mu} g_{\beta \nu}\right) x_{0}^{\alpha} x_{0}^{\mu} x_{1}^{\beta} x_{1}^{\nu}} .
$$

The Cartan form of the Lagrangian $\lambda$ is the 2 -form

$$
\begin{aligned}
\theta_{\lambda} & =L d \tau^{0} \wedge d \tau^{1}+\frac{\partial L}{\partial x_{0}^{\mu}} \omega^{\mu} \wedge d \tau_{1}-\frac{\partial L}{\partial x_{1}^{\mu}} \omega^{\mu} \wedge d \tau_{0} \\
& =T \sqrt{-D} d \tau^{0} \wedge d \tau^{1}+\frac{T}{\sqrt{-D}}\left(g_{\alpha \mu} g_{\beta \nu}-g_{\alpha \beta} g_{\mu \nu}\right) d x^{\mu} \wedge\left(x_{0}^{\alpha} x_{0}^{\beta} x_{1}^{\nu} d \tau^{0}-x_{1}^{\alpha} x_{1}^{\beta} x_{0}^{\nu} d \tau^{1}\right) .
\end{aligned}
$$

We introduce momenta

$$
\begin{aligned}
& p_{\mu}^{0}=\frac{\partial L}{\partial x_{0}^{\mu}}=-\frac{T}{\sqrt{-D}}\left(g_{\alpha \beta} g_{\mu \nu}-g_{\alpha \mu} g_{\beta \nu}\right) x_{0}^{\alpha} x_{1}^{\beta} x_{1}^{\nu}, \\
& p_{\mu}^{1}=\frac{\partial L}{\partial x_{1}^{\mu}}=-\frac{T}{\sqrt{-D}}\left(g_{\alpha \beta} g_{\mu \nu}-g_{\alpha \mu} g_{\beta \nu}\right) x_{1}^{\alpha} x_{0}^{\beta} x_{0}^{\nu},
\end{aligned}
$$

and notice that $p_{\mu}^{0} x_{0}^{\mu}=p_{\mu}^{1} x_{1}^{\mu}=-T \sqrt{-D}$, and $p_{\mu}^{0} x_{1}^{\mu}=p_{\mu}^{1} x_{0}^{\mu}=0$.

As we have seen, a projectable vector field $\Xi$ on $\boldsymbol{Y}$ is a symmetry of $\lambda$ if $\Xi$ satisfies the Noether equation $L_{J^{1} \Xi} \lambda=0$. The corresponding Noether current is then the horizontal 1-form $\left.\Psi=h\left(J^{1} \Xi\right\rfloor \theta_{\lambda}\right)$, and, by Noether theorem, it is closed along critical sections (on every extremal surface $\Sigma): d\left(\Psi \circ J^{1} \gamma\right)=0$.

To make explicit computations, denote

$$
\Xi=\xi^{i} \frac{\partial}{\partial \tau^{i}}+\Xi^{\mu} \frac{\partial}{\partial x^{\alpha}}, \quad J^{1} \Xi=\xi^{i} \frac{\partial}{\partial \tau^{i}}+\Xi^{\mu} \frac{\partial}{\partial x^{\mu}}+\left(d_{i} \Xi^{\mu}-x_{j}^{\mu} \frac{\partial \xi^{j}}{\partial \tau^{i}}\right) \frac{\partial}{\partial x_{i}^{\mu}} .
$$

Contracting $\theta_{\lambda}$ and taking the horizontal part we obtain

$$
\Psi=\Xi^{\mu}\left(p_{\mu}^{0} d \tau^{1}-p_{\mu}^{1} d \tau^{0}\right) .
$$

This means that to every symmetry $\Xi$ of $\lambda$ we have on shell a conservation law

$$
\Xi^{\mu}\left(\frac{\partial p_{\mu}^{0}}{\partial \tau^{0}}+\frac{\partial p_{\mu}^{1}}{\partial \tau^{1}}\right)=0
$$

Symmetries of our Lagrangian can be explicitly determined from the above Noether equation. Since $D$ does not depend on $\tau^{0}$ and $\tau^{1}$, with help of momenta the Noether equation yields

$$
T \frac{\partial \sqrt{-D}}{\partial x^{\mu}} \Xi^{\mu}-p_{\mu}^{i} d_{i} \Xi^{\mu}=0 .
$$

We can see that the equation does not contain the projection $\xi$ of $\Xi$ (which is a vector field on the base $\boldsymbol{X}=\mathbb{R}^{2}$ ). This means that every vector field on $\mathbb{R}^{2}$ is a symmetry of $\lambda$, in other words, we recover the fact that the action is reparametrization invariant. Now se can take into account only vertical symmetries (which are vector fields on the space-time $(M, g)$ ). If, moreover, $(M, g)$ is the Minkowski space-time, i.e., $\mathbb{R}^{4}$ with the metric $\operatorname{diag} g=(1,-1,-1,-1)$, then $D$ does not depend on the $x^{\mu}$ 's, and we immediately obtain that the Poincaré group is a symmetry group of $\lambda$. The symmetries and the corresponding Noether currents are $(s=1,2,3, \mu=0,1,2,3)$

$$
\begin{aligned}
& \Xi=\frac{\partial}{\partial x^{\mu}}, \quad \Psi=-p_{\mu}^{1} d \tau^{0}+p_{\mu}^{0} d \tau^{1}, \\
& \Xi=x^{s} \frac{\partial}{\partial x^{0}}+x^{0} \frac{\partial}{\partial x^{s}}, \quad \Psi=\left(-p_{0}^{1} x^{s}-p_{s}^{1} x^{0}\right) d \tau^{0}+\left(p_{0}^{0} x^{s}+p_{s}^{0} x^{0}\right) d \tau^{1}, \\
& \Xi=x^{2} \frac{\partial}{\partial x^{1}}-x^{1} \frac{\partial}{\partial x^{2}} \text { cycl, } \quad \Psi=\left(-p_{1}^{1} x^{2}+p_{2}^{1} x^{1}\right) d \tau^{0}+\left(p_{1}^{0} x^{2}-p_{2}^{0} x^{1}\right) d \tau^{1} .
\end{aligned}
$$




\subsection{Variational Lie derivative and cohomology}

The variational Lie derivative can be seen as a local differential operator which acts on cohomology classes trivializing them [84]. This fact has important consequences for symmetries and conservation laws associated with local variational problems generating global Euler-Lagrange expressions; see, e.g., [7, 25, 28].

As an immediate example of that feature, let $\delta(\cdot)$ denote a cohomology class defined by a closed class in the variational sequence, and let $\varepsilon$ be a dynamical form locally variational: $E_{n+1}(\varepsilon)=0$ (i.e., locally, on an open set $\boldsymbol{U}_{\iota}, \varepsilon \equiv E_{\lambda_{\iota}}=E_{n}\left(\lambda_{\iota}\right)$ ). In the case $q=n+1$, Theorem 4.10 implies that for every projectable vector field $\Xi$ on $\left.\boldsymbol{Y}, L_{J^{s} \Xi} \varepsilon=E_{n}\left(J^{s} \Xi_{V}\right\rfloor \varepsilon\right)$, that is, the Euler-Lagrange form of the global Lagrangian $\left.\lambda=J^{s} \Xi_{V}\right\rfloor \varepsilon$, which implies $\delta\left(L_{J^{s} \Xi} E_{\lambda_{\iota}}\right)=$ $\delta\left(E_{L_{J^{r} \Xi} \lambda_{\iota}}\right)=0$ even if the cohomology class of $E_{\lambda_{\iota}}$ is nontrivial. In particular, since $E_{L_{J}{ }^{r} \lambda_{\iota}} \equiv$ $\left.E_{n}\left(L_{J^{r} \Xi \lambda_{\iota}}\right)=E_{n}\left(J^{s} \Xi_{V}\right\rfloor E_{\lambda_{\iota}}\right)$ we see that Euler-Lagrange equations of the local problem defined by $L_{J^{r} \Xi \lambda_{\iota}}$ are the same as the Euler-Lagrange equations of the global problem defined by $\left.\lambda=J^{s} \Xi_{V}\right\rfloor E_{\lambda_{\iota}}$. Summarizing, a problem defined by a family of local Lagrangians $L_{J^{r} \Xi \lambda_{\iota}}$ is variationally equivalent to a global one $[84,89]$.

Analogous results can be obtained at any higher degree in the variational sequence. Another case of particular importance, the degree $n+2$, will be discussed below.

\subsection{Symmetries of Helmholtz forms and variational dynamical forms}

Invariance properties of classes in the variational sequence suggested to Krupka et al. $[56,57,58]$ the idea that there should be a close relationship between the notions of local variational triviality of a differential form and invariance of its exterior derivative. Namely, 'translating' Theorem 4.3 to the Takens representation, we get $[57,58]$ :

Theorem 5.2. The Lie derivative of a source $(n+k)$-form $\varepsilon$ by a contact symmetry $Z$ is locally variationally trivial if and only if the flow of $Z$ leaves invariant the source $(n+k+1)$-form $E_{n+k}(\varepsilon)$.

Applying this fact to $(n+1)$-forms we then get a rather surprising assertion:

Corollary $\mathbf{5 . 3}$ ([57, 58]). Contact symmetries of Helmholtz forms transfer nonvariational dynamical forms to locally variational ones.

This result has an interesting application: namely, to a system of equations which is not variational as it stands, and even does not possess any variational multiplier one can find a variational system related by a contact transformation Examples showing this interesting feature are elaborated in the papers $[57,58]$.

Furthermore, one can obtain interesting results in study of global properties of variational problems. Let us consider a variationally trivial Helmholtz-like form $\eta$, i.e., let $E_{n+2}(\eta)=0$, and denote the corresponding local system of dynamical forms by $\varepsilon_{\iota}$, i.e., let $\eta=E_{n+1}\left(\varepsilon_{\iota}\right)$. If a projectable vector field $\Xi$ on $Y$ is a symmetry of $\eta$ then, by the above, each of the local dynamical forms $\bar{\varepsilon}_{\iota}=L_{J^{s} \Xi} \varepsilon_{\iota}$ is locally variational (meaning that locally it is the Euler-Lagrange form of a Lagrangian). In fact, applying Theorem 4.10, case $k=2$, using the Takens representation with $\eta=R_{n+2}([\rho])$, and formula (4.6), we obtain

$$
\begin{aligned}
0 & \left.=L_{J^{s} \Xi} \eta=L_{J^{s} \Xi} E_{n+1}\left(\varepsilon_{\iota}\right)=E_{n+1}\left(L_{J^{s} \Xi} \varepsilon_{\iota}\right)=0+R_{n+2} \mathcal{E}_{n+1}\left(J^{s} \Xi_{V}\right\rfloor[\rho]\right) \\
& \left.\left.\left.=E_{n+1} R_{n+1}\left(J^{s} \Xi_{V}\right\rfloor[\rho]\right)=E_{n+1} R_{n+1}\left(\left[J^{s} \Xi_{V}\right\rfloor \eta\right]\right)=E_{n+1} \mathcal{I}\left(J^{s} \Xi_{V}\right\rfloor \eta\right),
\end{aligned}
$$

so that $\left.E_{n+1}\left(L_{J^{s} \Xi} \Xi \varepsilon_{\iota}\right)=E_{n+1} \mathcal{I}\left(J^{s} \Xi_{V}\right\rfloor \eta\right)=0$, meaning that the system of local Euler-Lagrange forms $L_{J^{s} \Xi \varepsilon_{\iota}}$ is variationally equivalent (in the sense that they have the same Helmholtz form) 
with the global dynamical form $\left.\varepsilon=\mathcal{I}\left(J^{s} \Xi_{V}\right\rfloor \eta\right)$. Moreover, $\varepsilon$ is locally variational, and therefore locally it is the Euler-Lagrange form of a local Lagrangian [85].

We conclude that the variational problem defined by a system of local Euler-Lagrange forms $\bar{\varepsilon}_{\iota}$ is variationally equivalent to the global variational problem defined by the EulerLagrange form $\varepsilon$. Remarkably, in mechanics this 'globalization procedure' means to add to the local equations a variational force, 'sewing the equations together' on overlaps of their domains.

\subsection{Generalized symmetries generating Noether currents}

Representing the variational sequence by differential forms is of fundamental importance for understanding the geometry of Noether theorems.

Let $\varepsilon=E_{\lambda_{\iota}}$ be a locally variational form on $J^{s} \boldsymbol{Y}$, and let a projectable vector field $\Xi$ on $\boldsymbol{Y}$ be a generalized symmetry, meaning that $L_{J^{s} \Xi} \varepsilon=0$. Recall that by the properties of the Lie derivative this means that $E_{n}\left(L_{J^{r} \Xi} \lambda_{\iota}\right)=0$, hence locally $L_{J^{r} \Xi} \lambda_{\iota}=d_{H} \beta_{\iota}$. On the other hand, by Theorem 4.10, $\left.L_{J^{s} \Xi} \varepsilon=E_{n}\left(J^{s} \Xi_{V}\right\rfloor \varepsilon\right)$, hence $\left.E_{n}\left(J^{s} \Xi_{V}\right\rfloor \varepsilon\right)=0$, and from here we have locally $\left.J^{s} \Xi_{V}\right\rfloor \varepsilon=d_{H} \nu_{\iota}$, where $\nu_{\iota}$ are local currents which are conserved on-shell (i.e., along critical sections). Denoting by $\epsilon_{\iota}$ the usual canonical Noether current defined by Proposition 4.7, case $q=n$, i.e., $\left.\left.\epsilon_{\iota}=J^{s} \Xi_{V}\right\rfloor p_{d_{V} \lambda_{\iota}}+J^{s} \Xi_{H}\right\rfloor \lambda_{\iota}$, and putting $\beta_{\iota}=\nu_{\iota}+\epsilon_{\iota}$, we can write $\left.J^{s} \Xi_{V}\right\rfloor \varepsilon+d_{H}\left(\epsilon_{\iota}-\beta_{\iota}\right)$ $=0$. We call the (local) current $\epsilon_{\iota}-\beta_{\iota}$ a Noether-Bessel-Hagen current. Thus Noether-BesselHagen currents $\epsilon_{\iota}-\beta_{\iota}$ are local currents associated with a generalized symmetry (conserved along critical sections); in [28] it was proved that a Noether-Bessel-Hagen current is variationally equivalent to a global current if and only if $\left.0=\delta_{n-1}\left(\Xi_{V}\right\rfloor \mathcal{E}_{n}\left(\lambda_{i}\right)\right) \in H^{1}\left(\boldsymbol{Y}\right.$, $\left.\operatorname{ker} d_{H}\right)$.

It is of interest to investigate under which conditions a Noether-Bessel-Hagen current is variationally equivalent to a Noether conserved current for a suitable Lagrangian. A NoetherBessel-Hagen current $\epsilon_{\lambda_{\iota}}-\beta_{\iota}$ associated with a generalized symmetry of $\varepsilon=E_{\lambda_{\iota}}$ is a Noether conserved current if it is of the form $\epsilon_{\lambda_{\iota}}-L_{J^{s} \Xi} \mu_{\iota}$, with $\mu_{\iota}$ a current satisfying $L_{J^{s} \Xi}\left(\lambda_{\iota}-d_{H} \mu_{\iota}\right)=0$ (i.e., $\Xi$ is a symmetry of the Lagrangian $\bar{\lambda}_{\iota}=\lambda_{\iota}-d_{H} \mu_{\iota}$ for $\varepsilon$ ) [87]. The reader can check that taking for an invariant Lagrangian instead of the canonical current $\left.J^{s} \Xi\right\rfloor \theta$ a current, related to a Lepage equivalent different than the Cartan form $\theta$, we get a current which provides the same conservation law, see [68]. We have the on shell conservation laws $d_{H}\left(\epsilon_{\lambda_{\iota}}-\beta_{\iota}\right)=0$. It can be proved that $\epsilon_{\lambda_{\iota}}-L_{J^{s} \Xi \mu_{\iota}}=\epsilon_{\lambda_{\iota}-d_{H} \mu_{\iota}}$ is not only closed but also exact on shell (for a detailed discussion see, e.g., [11]). In the following we shall give an example of application concerning symmetries of Jacobi type equations for a Chern-Simons $3 D$ gauge theory.

Example 5.4. Let us then consider the 3-dimensional Chern-Simons Lagrangian

$$
\lambda_{\mathrm{CS}}(A)=\frac{\kappa}{4 \pi} \epsilon^{i j m} \operatorname{Tr}\left(A_{i} d_{j} A_{m}+\frac{2}{3} A_{i} A_{j} A_{m}\right) \omega_{0}
$$

where $\omega_{0}$ is a 3 -dimensional volume density, $\kappa$ a constant, while $A_{i}=A_{i}^{\mu} J_{\mu}$ are the coefficients of the connection 1-form $A=A_{i} d x^{i}$ taking their values in any Lie algebra $\mathfrak{g}$ with generators $J_{\mu}$. By fixing $\mathfrak{g}=\mathfrak{s l}(2, \mathbb{R})$ and choosing the generators $J_{\nu}=\frac{1}{2} \sigma_{\nu}$, whith $\sigma_{\nu}$ Pauli matrices, we have $\left[J_{\mu}, J_{\nu}\right]=\eta^{\sigma \rho} \epsilon_{\rho \mu \nu} J_{\sigma}$ and $\operatorname{Tr}\left(J_{\mu} J_{\nu}\right)=\frac{1}{2} \eta_{\mu \nu}$, with $\eta=\operatorname{diag}(-1,1,1)$ and $\epsilon_{012}=1$. Hence, we can explicitly write $\lambda_{\mathrm{CS}}(A)=\frac{\kappa}{16 \pi} \epsilon^{i j k}\left(\eta_{\mu \nu} F_{i j}^{\mu} A_{\rho}^{\nu}-\frac{1}{3} \epsilon_{\mu \nu \rho} A_{i}^{\mu} A_{j}^{\nu} A_{k}^{\rho}\right) d s$, where $F_{i j}^{\mu}=$ $d_{i} A_{j}^{\mu}-d_{j} A_{i}^{\mu}+\epsilon_{\nu \rho}^{\mu} A_{i}^{\nu} A_{j}^{\rho}$ is the so-called field strength. Note that, while Chern-Simons equations of motion are covariant with respect to spacetime diffeomorphism and with respect to gauge transformations, the Chern-Simons Lagrangian, instead, is not gauge invariant. Indeed for a pure gauge tranformation generated by a vertical vector field $\xi$ - acting on the gauge potential as $L_{\xi} A_{i}^{\mu}=\nabla_{i} \xi^{\mu}$, where $\nabla_{i}$ is the covariant derivative with respect to a principal connection $\omega^{\mu}$ defined by $A^{\mu}$ in a usual way through the introduction of new variables [1] - we have $\mathcal{L}_{\xi} \lambda_{\mathrm{CS}}(A)$ $=d_{i}\left(\frac{\kappa}{8 \pi} \epsilon^{i j k} \eta_{\mu \nu} A_{j}^{\mu} d_{k} \xi^{\nu}\right)$. 
Let $\nu_{\iota}+\epsilon_{\iota}$ be a 0-cocycle of strong Noether currents for the Chern-Simons Lagrangian (here $\iota$ refers to an open set $U_{\iota}$ in a good covering and in the following we shall use the identification $\lambda_{\mathrm{CS}}(A)=\lambda_{\mathrm{CS}}(\iota)$ and use them indifferently); it is known [27] that if $j^{s} \Xi$ is a symmetry of the Chern-Simons dynamical form, the global conserved current

$$
\left.\left.\Xi_{H}\right\rfloor \mathcal{L}_{\Xi} \lambda_{\mathrm{CS}_{\iota}}+j^{s} \Xi_{V}\right\rfloor p_{d_{V} \mathcal{L}_{\Xi} \lambda_{\mathrm{CS} \iota}}
$$

is associated with the invariance of the Chern-Simons equations and it is variationally equivalent to the variation of the strong Noether currents $\nu_{\iota}+\epsilon_{\iota}$. Generators of such a global current lie in the kernel of the second variational derivative and are symmetries of the variationally trivial Lagrangian $\mathcal{L}_{j^{s} \Xi_{V}} \lambda_{\mathrm{CS}}(\iota)$.

Let now require $j^{s} \Xi$ to be a symmetry of the Euler-Lagrange class of the 'deformed' Lagrangian $\mathcal{L}_{j^{s} \Xi} \lambda_{\mathrm{CS}}(\iota)$, but not of the Chern-Simons class $E_{n}\left(\lambda_{\mathrm{CS}}(\iota)\right.$ ) (this means that $j^{s} \Xi$ is not

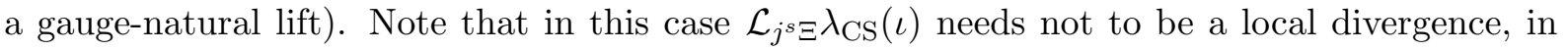
general. By naturality and by Theorem 4.10 this means that we require

$$
\mathcal{L}_{j^{s} \Xi} E_{n}\left(\Xi_{V}\right\rfloor E_{n}\left(\lambda_{\mathrm{CS}}(\iota)\right)=0,
$$

i.e., $j^{s} \Xi$ is a symmetry of a Jacobi type dynamical form (or Euler-Lagrange class of the deformed Lagrangian). Let now $j^{s} \Xi_{V}$ be the generator of a gauge transformation. Since we know that $\mathcal{L}_{j^{s} \Xi_{V}} \lambda_{\mathrm{CS}}(\iota)=d_{H} \gamma$, it is clear that such a $j^{s} \Xi$ is a Noether symmetry of the Lagrangian

$$
\left.\Xi_{V}\right\rfloor E_{n}\left(\lambda_{\mathrm{CS}}(\iota)-d_{H}\left(\gamma-j^{s} \Xi_{V}\right\rfloor p_{d_{V} \lambda_{\mathrm{CS}}}\right),
$$

this comes from equation (5.2) and from

$$
\left.\mathcal{L}_{j^{s} \Xi}\left(\mathcal{L}_{j^{s} \Xi} \lambda_{\mathrm{CS}}(\iota)-d_{H} \epsilon_{\lambda_{\mathrm{CS}}}\right)=\mathcal{L}_{j^{s} \Xi}\left(d_{H} \gamma-d_{H}\left(\Xi_{V}\right\rfloor p_{d_{V} \lambda_{\mathrm{CS}}}\right)\right) .
$$

By applying the above results concerning Noether-Bessel-Hagen currents to the 'deformed' Lagrangian we can then state that along sections pulling back to zero the Jacobi type dynamical form (note that solutions of Chern-Simons equations are among such sections) we have that

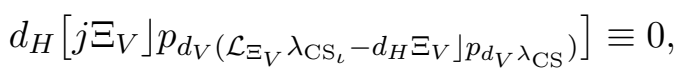

i.e., the corresponding Noether-Bessel-Hagen current must be exact on shell which implies a constraint for the connection 1-form $A$.

\subsection{Sharp obstruction to the existence of global solutions in 3D Chern-Simons theories}

We now explore the relationship between the existence of global critical sections and the existence of global Noether-Bessel-Hagen currents. We denote by [[- $]]_{\mathrm{dR}}$ a class in de Rham cohomology. As already recalled, the Noether-Bessel-Hagen current is variationally equivalent to a global current if and only if $\left.\left.0=\delta_{n-1}\left(\Xi_{V}\right\rfloor \varepsilon\right) \sim\left[\left[\Xi_{V}\right\rfloor \varepsilon\right]\right]_{\mathrm{dR}} \in H_{\mathrm{dR}}^{n}(\boldsymbol{Y})$.

In the following we shall need to see certain cohomology classes in $H_{\mathrm{dR}}^{n}(\boldsymbol{Y})$ as the pull-back of cohomology classes in $H_{\mathrm{dR}}^{n}(\boldsymbol{X})$, i.e., defined by closed differential forms on the base manifold.

Let us then assume $\sigma$ be a global section of $\pi: \boldsymbol{Y} \rightarrow \boldsymbol{X}$; for any global section we have of course $\sigma^{*} \circ \pi^{*}=\operatorname{id}_{H_{\mathrm{dR}}^{n}(\boldsymbol{X})}$. Let furthermore $H_{\mathrm{dR}}^{n}(\boldsymbol{Y}) \sim \pi^{*}\left(H_{\mathrm{dR}}^{n}(\boldsymbol{X})\right)$, i.e., the pull-back $\pi^{*}: H_{\mathrm{dR}}^{n}(\boldsymbol{X}) \rightarrow$ $H_{\mathrm{dR}}^{n}(\boldsymbol{Y})$ is an isomorphism of cohomology groups.

We need to compare the cohomology of the base manifold with the cohomology of the total space, and while $H_{\mathrm{dR}}^{n}(\boldsymbol{Y}) \simeq H_{V S}^{n}(\boldsymbol{Y})$, in principle we do not have a similar isomorphism for the cohomology groups $H_{\mathrm{dR}}^{n}(\boldsymbol{X})$. This is overcome by the peculiar structure of quotient space in the variational sequence: since for all sections $J^{r} \sigma^{*}\left(\Theta_{*}^{r}\right)=0$, we can correctly define 
$\left.\left.J^{r} \sigma^{*}\left[\left[\Xi_{V}\right\rfloor \varepsilon\right]\right]_{\mathrm{dR}} \doteq\left[\left[J^{r} \sigma^{*}\left(\Xi_{V}\right\rfloor \varepsilon\right)\right]\right]_{\mathrm{dR}} ;$ in other words, since $\Theta_{n}^{r}$ consists of contact $n$-forms the pullback $\sigma^{*}$ factorizes through $\Lambda_{n}^{r} / \Theta_{n}^{r}$. Furthermore, there always exists a closed form $\alpha \in \Lambda_{n}^{r}$, e.g., a Lepage equivalent, which represents the cohomology class $\left.\left[\left[\Xi_{V}\right\rfloor \varepsilon\right]\right]_{\mathrm{dR}}$ and which projects onto $\left.\Xi_{V}\right\rfloor \varepsilon \in \Lambda_{n}^{r} / \Theta_{n}^{r}$. Thus, if $\sigma$ is a critical section, $J^{r} \sigma^{*}(\alpha)=0$ and the corresponding class vanishes in $H_{\mathrm{dR}}^{n}(\boldsymbol{X})$; therefore, by our assumption on the $n$th cohomology groups $\left.\left[\left[\Xi_{V}\right\rfloor \varepsilon\right]\right]_{\mathrm{dR}}=0$ and there exist global Noether-Bessel-Hagen conserved currents for all generalized symmetries $\Xi[28]$.

We can use the result above also conversely to define a cohomological obstruction to the existence of global solutions for a given problem. Let then $\sigma$ be a section of $\boldsymbol{Y}$ over $\boldsymbol{X}$. Let $\alpha$ be a closed differential form which represents $\left.\left[\left[\Xi_{V}\right\rfloor \varepsilon\right]\right]_{\mathrm{dR}} \in H_{\mathrm{dR}}^{n}(\boldsymbol{Y})$, the obstruction to the existence of global conserved quantities for conservation laws associated with the problem $\varepsilon$ and its symmetry $\Xi$. Since $\sigma$ (as well as any other section) defines a projection $\sigma^{*}: H_{\mathrm{dR}}^{n}(\boldsymbol{Y}) \mapsto$ $H_{\mathrm{dR}}^{n}(\boldsymbol{X})$, if the class $J^{r} \sigma^{*}[[\alpha]]_{\mathrm{dR}} \in H_{\mathrm{dR}}^{n}(\boldsymbol{X})$ does not vanish, then there are no global solutions.

In the case we drop the above condition on the isomorphism of cohomology groups, if the pull-back with a given section of the cohomology class $\left.\left[\left[\Xi_{V}\right\rfloor \varepsilon\right]\right]_{\mathrm{dR}}$ is not zero, no other homotopic section can be a critical section. Note that, in this case, the obstruction does depend on the section; $J^{r} \sigma^{*}[[\alpha]]_{\mathrm{dR}}$ may vanish for sections from one homotopy class, but not for those from another [28].

It is also important to note that this obstruction is not identically zero, in general.

As an example, let us consider the classical 3-dimensional Chern-Simons theory. It is a classical field theory for principal connections on an arbitrary principal bundle $\boldsymbol{P}$ over a 3 -dimensional manifold $\boldsymbol{M}$. It is well known that the equations can be written $F=0$ ( $F$ the curvature); these equations admit solutions if and only if the cohomology class $\left[\left[\operatorname{tr} F_{A}\right]\right]_{\mathrm{dR}}$ ("first Chern class") vanishes for an arbitrary (and, thus, for every) connection $A$. Thus, we will consider only principal bundles $\boldsymbol{P}$ with group U(1) or $\mathbb{C}^{*}$ (taking the trace of the curvature corresponds to "taking the determinant of $\boldsymbol{P} ")$.

To apply our result, we have to formulate Chern-Simons theory in terms of the bundle of connections: the bundle of connections is defined as

$$
\mathcal{C}:=J^{1} \boldsymbol{P} / \boldsymbol{G} \mapsto \boldsymbol{P} / \boldsymbol{G} \sim \boldsymbol{X},
$$

the principal connections on $\boldsymbol{P}$ are in one to one correspondence with the (global) sections of the bundle $\mathcal{C} \mapsto \boldsymbol{X}$; Chern-Simons theory can then be formulated as a variational theory on $J^{1} \mathcal{C}$. In this case is it advantageous to lift the dynamical form of the problem to the de Rham complex: we can find a differential form $\Sigma$ on $J^{1} \mathcal{C}$ such that a section $\sigma$ of $\mathcal{C} \mapsto \boldsymbol{X}$ is critical if and only if

$$
\left.J^{1} \sigma^{*}(\Xi\rfloor \Sigma\right)=0,
$$

for all vector fields $\Xi$ on $J^{1} \mathcal{C}$.

We have that the contact structure of $J^{1} \boldsymbol{P}$ defines a connection on the principal bundle $J^{1} \boldsymbol{P} \mapsto \mathcal{C}$; its curvature is given by $\mathcal{F}=d A_{j} \wedge d x^{j}$ (structure group U(1) or $\mathbb{C}^{*}$ ). This connection is universal in the sense that every principal connection on $\boldsymbol{P} \mapsto \boldsymbol{X}$ is "induced" by it via a section $\sigma: X \mapsto \mathcal{C}$, in particular $F_{\sigma}=\sigma^{*} \mathcal{F}$.

We can set now

$$
\Sigma:=\pi_{1,0}^{*} \mathcal{F} \wedge \mathcal{F},
$$

where $\Sigma$ is, of course, the exterior differential of the Poincaré-Cartan form. Notice that a projectable vector field $\Xi$ is a symmetry of $\Sigma$ if and only if $d(\Xi\rfloor \Sigma)=0$. The corresponding class to $\left.\left[\left[\Xi_{V}\right\rfloor \varepsilon\right]\right]_{\mathrm{dR}}$ for a generic $\varepsilon$ is now $\left.\left.[[\Xi\rfloor \Sigma]\right]_{\mathrm{dR}}=2 \pi_{1,0}^{*}((\Xi\rfloor \mathcal{F}) \wedge \mathcal{F}\right)$.

If the Euler-Lagrange equations admit global solutions, then $\mathcal{F}=d \gamma$ and, thus,

$$
\left.[[\Xi\rfloor \Sigma]]_{\mathrm{dR}}=2 \pi_{1,0}^{*}((\Xi\rfloor \mathcal{F}) \wedge d \gamma\right)=2 d\left(\pi_{1,0}^{*}(\Xi\rfloor \mathcal{F} \wedge \gamma\right) .
$$

If the equations do not admit global solutions then $[[\mathcal{F}]]_{\mathrm{dR}} \neq 0$. 
Now, if $\boldsymbol{X}$ is a closed manifold, then there is a closed cohomologically nontrivial 1-form $\alpha$ such that $\alpha \wedge F_{\sigma}$ and, hence, $\left(\pi^{*}(\alpha)\right) \wedge \mathcal{F}$ are cohomologically non trivial (Poincaré duality).

Since $\boldsymbol{X}$ is parallelizable, we can easily find a vertical vector field $\Phi$ such that $\Phi\rfloor \mathcal{F}=\pi^{*}(\alpha)$ and

$$
[[\Phi\rfloor \Sigma]]_{\mathrm{dR}}=2 \pi_{1,0}^{*}\left(\pi^{*}(\alpha) \wedge \mathcal{F}\right) .
$$

Thus, for closed 3-manifolds Chern-Simons theories admit solutions if and only if

$$
[[\Phi\rfloor \Sigma]]_{\mathrm{dR}}=0 .
$$

For non compact manifolds this is, of course, no longer true; thus, for non compact 3-manifolds the question, whether the obstruction is sharp, depends on the existence of a suitable compactification, see also [88].

\section{Acknowledgements}

Research supported by Department of Mathematics, University of Torino, research projects Geometric methods in mathematical physics and applications 2014 and 2015, GNSAGA-INdAM and by grant no 14-02476S Variations, Geometry and Physics of the Czech Science Foundation. MP and EW are grateful to M. Ferraris for useful discussions. OR also wishes to acknowledge travel support by the IRSES project LIE-DIFF-GEOM (EU FP7, nr 317721) and the hospitality of the Department of Mathematics, Ghent University, Belgium.

\section{References}

[1] Allemandi G., Francaviglia M., Raiteri M., Covariant charges in Chern-Simons AdS 3 gravity, Classical Quantum Gravity 20 (2003), 483-506, gr-qc/0211098.

[2] Anderson I.M., The variational bicomplex, Technical report of the Utah State University, 1989.

[3] Anderson I.M., Duchamp T., On the existence of global variational principles, Amer. J. Math. 102 (1980), $781-868$.

[4] Bauderon M., Le problème inverse du calcul des variations, Ann. Inst. H. Poincaré Sect. A 36 (1982), 159-179.

[5] Bessel-Hagen E., Über die Erhaltungssätze der Elektrodynamik, Math. Ann. 84 (1921), 258-276.

[6] Betounes D.E., Extension of the classical Cartan form, Phys. Rev. D 29 (1984), 599-606.

[7] Borowiec A., Ferraris M., Francaviglia M., Palese M., Conservation laws for non-global Lagrangians, Univ. Iagel. Acta Math. (2003), 319-331, math-ph/0301043.

[8] Bredon G.E., Sheaf theory, McGraw-Hill Book Co., New York - Toronto, Ont. - London, 1967.

[9] Carathéodory C., Über die Variationsrechnung bei mehrfachen Integralen, Acta Litt. Sci. Szeged 4 (1929), $193-216$.

[10] Cartan E., Lecons sur les invariants intègraux, Hermann, Paris, 1922.

[11] Cattafi F., Palese M., Winterroth E., Variational derivatives in locally Lagrangian field theories and Noether-Bessel-Hagen currents, Int. J. Geom. Methods Mod. Phys. 13 (2016), 1650067, 16 pages, arXiv:1601.07193.

[12] De Donder Th., Théorie invariantive du calcul des variations, Gauthier-Villars, Paris, 1930.

[13] de León M., Rodrigues P.R., Generalized classical mechanics and field theory. A geometrical approach of Lagrangian and Hamiltonian formalisms involving higher order derivatives, North-Holland Mathematics Studies, Vol. 112, North-Holland Publishing Co., Amsterdam, 1985.

[14] Dedecker P., Systèmes différentiels extérieurs, invariants intégraux et suites spectrales, in Convegno Internazionale di Geometria Differenziale (Italia, 1953), Edizioni Cremonese, Roma, 1954, 247-262. 
[15] Dedecker P., Quelques applications de la suite spectrale aux intégrales multiples du calcul des variations et aux invariants intégraux. I. Suites spectrales et généralisations, Bull. Soc. Roy. Sci. Liège 24 (1955), $276-295$.

[16] Dedecker P., Quelques applications de la suite spectrale aux intégrales multiples du calcul des variations et aux invariants intégraux. II. Espaces de jets locaux et faisceaux, Bull. Soc. Roy. Sci. Liège 25 (1956), $387-399$.

[17] Dedecker P., Calcul des variations et topologie algébrique, Mém. Soc. Roy. Sci. Liège 19 (1957), 216 pages.

[18] Dedecker P., On the generalization of symplectic geometry to multiple integrals in the calculus of variations, in Differential Geometrical Methods in Mathematical Physics (Proc. Sympos., Univ. Bonn, Bonn, 1975), Lecture Notes in Math., Vol. 570, Springer, Berlin, 1977, 395-456.

[19] Dedecker P., Tulczyjew W.M., Spectral sequences and the inverse problem of the calculus of variations, in Differential Geometrical Methods in Mathematical Physics (Proc. Conf., Aix-en-Provence/Salamanca, 1979), Lecture Notes in Math., Vol. 836, Springer, Berlin, 1980, 498-503.

[20] Dickey L.A., On exactness of the variational bicomplex, in Mathematical Aspects of Classical Field Theory (Seattle, WA, 1991), Contemp. Math., Vol. 132, Editors M. Gotay, J. Marsden, V. Moncrief, Amer. Math. Soc., Providence, RI, 1992, 307-315.

[21] Ferraris M., Fibered connections and global Poincaré-Cartan forms in higher-order calculus of variations, in Proceedings of the Conference on Differential Geometry and its Applications, Part 2, Editor D. Krupka, J.E. Purkyně University, Brno, 1984, 61-91.

[22] Ferraris M., Francaviglia M., On the global structure of Lagrangian and Hamiltonian formalisms in higher order calculus of variations, in Proceedings of the International Meeting on Geometry and Physics (Florence, 1982), Editor M. Modugno, Pitagora, Bologna, 1983, 43-70.

[23] Ferraris M., Francaviglia M., Palese M., Winterroth E., Canonical connections in gauge-natural field theories, Int. J. Geom. Methods Mod. Phys. 5 (2008), 973-988.

[24] Ferraris M., Francaviglia M., Palese M., Winterroth E., Gauge-natural Noether currents and connection fields, Int. J. Geom. Methods Mod. Phys. 8 (2011), 177-185.

[25] Ferraris M., Palese M., Winterroth E., Local variational problems and conservation laws, Differential Geom. Appl. 29 (2011), S80-S85.

[26] Francaviglia M., Palese M., Vitolo R., Symmetries in finite order variational sequences, Czechoslovak Math. J. 52 (2002), 197-213.

[27] Francaviglia M., Palese M., Winterroth E., Locally variational invariant field equations and global currents: Chern-Simons theories, Commun. Math. 20 (2012), 13-22.

[28] Francaviglia M., Palese M., Winterroth E., Variationally equivalent problems and variations of Noether currents, Int. J. Geom. Methods Mod. Phys. 10 (2013), 1220024, 10 pages, arXiv:1512.03176.

[29] García P.L., The Poincaré-Cartan invariant in the calculus of variations, in Symposia Mathematica, Vol. XIV (Convegno di Geometria Simplettica e Fisica Matematica, INDAM, Rome, 1973), Academic Press, London, 1974, 219-246.

[30] García P.L., Muñoz J., On the geometrical structure of higher order variational calculus, Atti Accad. Sci. Torino Cl. Sci. Fis. Mat. Natur. 117 (1983), suppl. 1, 127-147.

[31] Gel'fand I.M., Dikii L.A., Asymptotic properties of the resolvent of Sturm-Liouville equations, and the algebra of Korteweg-de Vries equations, Russian Math. Surveys 30 (1975), no. 5, 77-113.

[32] Goldschmidt H., Sternberg S., The Hamilton-Cartan formalism in the calculus of variations, Ann. Inst. Fourier (Grenoble) $\mathbf{2 3}$ (1973), 203-267.

[33] Gotay M.J., An exterior differential systems approach to the Cartan form, in Symplectic Geometry and Mathematical Physics (Aix-en-Provence, 1990), Progr. Math., Vol. 99, Birkhäuser Boston, Boston, MA, 1991, 160-188.

[34] Gotay M.J., A multisymplectic framework for classical field theory and the calculus of variations. I. Covariant Hamiltonian formalism, in Mechanics, Analysis and Geometry: 200 Years after Lagrange, Editors M. Francaviglia, D.D. Holm, North-Holland Delta Ser., North-Holland, Amsterdam, 1991, 203-235.

[35] Gotô T., Relativistic quantum mechanics of one-dimensional mechanical continuum and subsidiary conditon of dual resonance model, Progr. Theoret. Phys. 46 (1971), 1560-1569.

[36] Grigore D.R., The variational sequence on finite jet bundle extensions and the Lagrangian formalism, Differential Geom. Appl. 10 (1999), 43-77, dg-ga/9702016. 
[37] Grigore D.R., On a generalization of the Poincaré-Cartan form in higher-order field theory, in Variations, Geometry and Physics, Editors O. Krupková, D.J. Saunders, Nova Sci. Publ., New York, 2009, 57-76.

[38] Haková A., Krupková O., Variational first-order partial differential equations, J. Differential Equations 191 (2003), 67-89.

[39] Helmholtz H., Ueber die physikalische Bedeutung des Prinicips der kleinsten Wirkung, J. Reine Angew. Math. 100 (1887), 137-166.

[40] Horák M., Kolář I., On the higher order Poincaré-Cartan forms, Czechoslovak Math. J. 33 (1983), $467-475$.

[41] Horndeski G.W., Differential operators associated with the Euler-Lagrange operator, Tensor (N.S.) 28 (1974), 303-318.

[42] Kolář I., A geometrical version of the higher order Hamilton formalism in fibred manifolds, J. Geom. Phys. 1 (1984), 127-137.

[43] Krbek M., The representation of the variational sequence by forms, Ph.D. Thesis, University of Brno, 2002.

[44] Krbek M., Musilová J., Representation of the variational sequence by differential forms, Rep. Math. Phys. 51 (2003), 251-258.

[45] Krbek M., Musilová J., Representation of the variational sequence by differential forms, Acta Appl. Math. 88 (2005), 177-199.

[46] Krupka D., Some geometric aspects of variational problems in fibred manifolds, Folia Fac. Sci. Nat. UJEP Brunensis Physica 14 (1973), 1-65, math-ph/0110005.

[47] Krupka D., A map associated to the Lepagian forms on the calculus of variations in fibred manifolds, Czechoslovak Math. J. 27 (1977), 114-118.

[48] Krupka D., Lepagean forms in higher order variational theory, Atti Accad. Sci. Torino Cl. Sci. Fis. Mat. Natur. 117 (1983), suppl. 1, 197-238.

[49] Krupka D., Variational sequences on finite order jet spaces, in Differential Geometry and its Applications (Brno, 1989), Editors J. Janyška, D. Krupka, World Sci. Publ., Teaneck, NJ, 1990, 236-254.

[50] Krupka D., The contact ideal, Differential Geom. Appl. 5 (1995), 257-276.

[51] Krupka D., Variational sequences in mechanics, Calc. Var. Partial Differential Equations 5 (1997), 557-583.

[52] Krupka D., Variational sequences and variational bicomplexes, in Differential Geometry and Applications (Brno, 1998), Editors I. Kolár, O. Kowalski, D. Krupka, J. Slovák, Masaryk University, Brno, 1999, 525-531.

[53] Krupka D., Global variational principles: foundations and current problems, in Global Analysis and Applied Mathematics, AIP Conf. Proc., Vol. 729, Amer. Inst. Phys., Melville, NY, 2004, 3-18.

[54] Krupka D., Global variational theory in fibred spaces, inHandbook of Global Analysis, Elsevier Sci. B. V., Amsterdam, 2008, 773-836.

[55] Krupka D., Introduction to global variational geometry, Atlantis Studies in Variational Geometry, Vol. 1, Atlantis Press, Paris, 2015.

[56] Krupka D., Krupková O., Contact symmetries and variational PDE's, Acta Appl. Math. 101 (2008), 163-176.

[57] Krupka D., Krupková O., Prince G., Sarlet W., Contact symmetries and variational sequences, in Differential Geometry and its Applications, Matfyzpress, Prague, 2005, 605-615.

[58] Krupka D., Krupková O., Prince G., Sarlet W., Contact symmetries of the Helmholtz form, Differential Geom. Appl. 25 (2007), 518-542.

[59] Krupka D., Krupková O., Saunders D., The Cartan form and its generalizations in the calculus of variations, Int. J. Geom. Methods Mod. Phys. 7 (2010), 631-654.

[60] Krupka D., Krupková O., Saunders D., Cartan-Lepage forms in geometric mechanics, Internat. J. NonLinear Mech. 47 (2012), 1154-1160.

[61] Krupka D., Šeděnková J., Variational sequences and Lepage forms, in Differential Geometry and its Applications, Editors J. Bureš, O. Kowalski, D. Krupka, J. Slovák, Matfyzpress, Prague, 2005, 617-627.

[62] Krupka D., Trautman A., General invariance of Lagrangian structures, Bull. Acad. Polon. Sci. Sér. Sci. Math. Astronom. Phys. 22 (1974), 207-211.

[63] Krupka D., Urban Z., Volná J., Variational projectors in fibred manifolds, Miskolc Math. Notes 14 (2013), 503-516.

[64] Krupková O., Lepagean 2-forms in higher order Hamiltonian mechanics. I. Regularity, Arch. Math. (Brno) 22 (1986), 97-120. 
[65] Krupková O., The geometry of ordinary variational equations, Lecture Notes in Math., Vol. 1678, SpringerVerlag, Berlin, 1997.

[66] Krupková O., Hamiltonian field theory, J. Geom. Phys. 43 (2002), 93-132.

[67] Krupková O., Lepage forms in the calculus of variations, in Variations, Geometry and Physics, Nova Sci. Publ., New York, 2009, 27-55.

[68] Krupková O., Variational equations on manifolds, in Advances in Mathematics Research, Adv. Math. Res., Vol. 9, Nova Sci. Publ., New York, 2009, 201-274.

[69] Krupková O., Malíková R., Helmholtz conditions and their generalizations, Balkan J. Geom. Appl. 15 (2010), 80-89.

[70] Krupková O., Prince G.E., Lepagean forms, closed 2-forms, and second-order ordinary differential equations, Russian Math. 51 (2007), no. 12, 1-16.

[71] Krupková O., Prince G.E., Second order ordinary differential equations in jet bundles and the inverse problem of the calculus of variations, in Handbook of Global Analysis, Elsevier Sci. B. V., Amsterdam, 2008, 837-904.

[72] Krupková O., Smetanová D., Lepage equivalents of second-order Euler-Lagrange forms and the inverse problem of the calculus of variations, J. Nonlinear Math. Phys. 16 (2009), 235-250.

[73] Lepage Th.H.J., Sur les champ geodesiques du calcul de variations. I, Bull. Acad. Roy. Belg. Cl. Sci. 22 (1936), 716-729.

[74] Lepage Th.H.J., Sur les champ geodesiques du calcul de variations. II, Bull. Acad. Roy. Belg. Cl. Sci. 22 (1936), 1036-1046.

[75] Malíková R., On a generalization of Helmholtz conditions, Acta Math. Univ. Ostrav. 17 (2009), 11-21.

[76] Musilová J., Lenc M., Lepage forms in variational theories: from Lepage's idea to the variational sequence, in Variations, Geometry and Physics, Nova Sci. Publ., New York, 2009, 3-26.

[77] Noether E., Invariante Variationsprobleme, Nachr. Ges. Wiss. Göttingen Math.-Phys. Kl. (1918), $235-257$.

[78] Olver P.J., Equivalence and the Cartan form, Acta Appl. Math. 31 (1993), 99-136.

[79] Olver P.J., Shakiban C., A resolution of the Euler operator. I, Proc. Amer. Math. Soc. 69 (1978), 223-229.

[80] Palese M., Vitolo R., On a class of polynomial Lagrangians, Rend. Circ. Mat. Palermo (2001), suppl. 66, 147-159, math-ph/0111019.

[81] Palese M., Winterroth E., Global generalized Bianchi identities for invariant variational problems on gaugenatural bundles, Arch. Math. (Brno) 41 (2005), 289-310, math-ph/0311003.

[82] Palese M., Winterroth E., On the relation between the Jacobi morphism and the Hessian in gauge-natural field theories, Theoret. and Math. Phys. 152 (2007), 1191-1200.

[83] Palese M., Winterroth E., Lagrangian reductive structures on gauge-natural bundles, Rep. Math. Phys. 62 (2008), 229-239, arXiv:0712.0925.

[84] Palese M., Winterroth E., A variational perspective on classical Higgs fields in gauge-natural theories, Theoret. and Math. Phys. 168 (2011), 1002-1008, arXiv:1110.5426.

[85] Palese M., Winterroth E., Symmetries of Helmholtz forms and globally variational dynamical forms, J. Phys. Conf. Ser. 343 (2012), 012129, 4 pages, arXiv:1110.5764.

[86] Palese M., Winterroth E., Higgs fields on spinor gauge-natural bundles, J. Phys. Conf. Ser. 411 (2013), 012025, 5 pages.

[87] Palese M., Winterroth E., Generalized symmetries generating Noether currents and canonical conserved quantities, J. Phys. Conf. Ser. 563 (2014), 012023, 4 pages.

[88] Palese M., Winterroth E., Topological obstructions in Lagrangian field theories (with an application to Chern-Simons gauge theories), in preparation.

[89] Palese M., Winterroth E., Garrone E., Second variational derivative of local variational problems and conservation laws, Arch. Math. (Brno) 47 (2011), 395-403.

[90] Rund H., A Cartan form for the field theory of Carathéodory in the calculus of variations of multiple integrals, in Differential Geometry, Calculus of Variations, and their Applications, Lecture Notes in Pure and Appl. Math., Vol. 100, Dekker, New York, 1985, 455-469.

[91] Saunders D.J., The geometry of jet bundles, London Mathematical Society Lecture Note Series, Vol. 142, Cambridge University Press, Cambridge, 1989. 
[92] Saunders D.J., The Cartan form, 20 years on, in Differential Geometry and its Applications, Editors O. Kowalski, D. Krupka, O. Krupková, J. Slovák, World Sci. Publ., Hackensack, NJ, 2008, 527-537.

[93] Takens F., A global version of the inverse problem of the calculus of variations, J. Differential Geom. 14 (1979), 543-562.

[94] Tonti E., Variational formulation of nonlinear differential equations. I, Acad. Roy. Belg. Bull. Cl. Sci. 55 (1969), 137-165.

[95] Tonti E., Variational formulation of nonlinear differential equations. II, Acad. Roy. Belg. Bull. Cl. Sci. 55 (1969), 262-278.

[96] Tulczyjew W.M., The Lagrange complex, Bull. Soc. Math. France 105 (1977), 419-431.

[97] Tulczyjew W.M., The Euler-Lagrange resolution, in Differential Geometrical Methods in Mathematical Physics (Proc. Conf., Aix-en-Provence/Salamanca, 1979), Lecture Notes in Math., Vol. 836, Springer, Berlin, 1980, 22-48.

[98] Tulczyjew W.M., Cohomology of the Lagrange complex, Ann. Scuola Norm. Sup. Pisa Cl. Sci. 14 (1987), $217-227$.

[99] Vinogradov A.M., On the algebro-geometric foundations of Lagrangian field theory, Soviet Math. Dokl. 18 (1977), 1200-1204.

[100] Vinogradov A.M., The $\mathcal{C}$-spectral sequence, Lagrangian formalism, and conservation laws. I. The linear theory, J. Math. Anal. Appl. 100 (1984), 1-40.

[101] Vinogradov A.M., The $\mathcal{C}$-spectral sequence, Lagrangian formalism, and conservation laws. II. The nonlinear theory, J. Math. Anal. Appl. 100 (1984), 41-129.

[102] Vitolo R., Variational sequences, in Handbook of Global Analysis, Elsevier Sci. B. V., Amsterdam, 2008, $1115-1163$.

[103] Volná J., Urban Z., The interior Euler-Lagrange operator in field theory, Math. Slovaca 65 (2015), 14271444.

[104] Wells Jr. R.O., Differential analysis on complex manifolds, Graduate Texts in Mathematics, Vol. 65, 2nd ed., Springer-Verlag, New York - Berlin, 1980. 